\title{
A Recoverable Mutex Algorithm with Sub-logarithmic RMR on Both CC and DSM
}

\author{
Prasad Jayanti* $\quad$ Siddhartha Jayanti ${ }^{\dagger} \quad$ Anup Joshi ${ }^{\ddagger}$
}

\begin{abstract}
In light of recent advances in non-volatile main memory technology, Golab and Ramaraju reformulated the traditional mutex problem into the novel Recoverable Mutual Exclusion (RME) problem. In the best known solution for RME, due to Golab and Hendler from PODC 2017, a process incurs at most $O\left(\frac{\log n}{\log \log n}\right)$ remote memory references (RMRs) per passage, where a passage is an interval from when a process enters the Try section to when it subsequently returns to Remainder. Their algorithm, however, guarantees this bound only for cache-coherent (CC) multiprocessors, leaving open the question of whether a similar bound is possible for distributed shared memory (DSM) multiprocessors.

We answer this question affirmatively by designing an algorithm that satisfies the same complexity bound as Golab and Hendler's for both CC and DSM multiprocessors. Our algorithm has some additional advantages over Golab and Hendler's: (i) its Exit section is wait-free, (ii) it uses only the Fetch-and-Store instruction, and (iii) on a CC machine our algorithm needs each process to have a cache of only $O(1)$ words, while their algorithm needs $O(n)$ words .
\end{abstract}

\section{Introduction}

In light of recent advances in non-volatile main memory technology, Golab and Ramaraju reformulated the traditional mutex problem into the novel Recoverable Mutual Exclusion (RME) problem. The best known algorithm for RME, due to Golab and Hendler [5], has sub-logarithmic remote memory reference (RMR) complexity for Cache-Coherent (CC) multiprocessors, but unbounded RMR complexity for Distributed Shared Memory (DSM) multiprocessors. In this paper, we present an algorithm that ensures the same sublogarithmic bound as theirs for both CC and DSM multiprocessors, besides possessing some additional desirable properties. In the rest of this section, we describe the model, the RME problem, the complexity measure, and then describe this paper's contribution in the context of prior work.

\section{$1.1 \quad$ The Model}

The advent of Non-Volatile Random Access Memory (NVRAM) [13]14] 15] - memory whose contents remain intact despite process crashes - has led to a new and natural model of a multiprocessor and spurred research on the design of algorithms for this model. In this model, asynchronous processes communicate by applying operations on shared variables stored in an NVRAM. A process may crash from time to time. When a process $\pi$ crashes, all of $\pi$ 's registers lose their contents: specifically, $\pi$ 's program counter is reset to point to a default location $\ell$ in $\pi$ 's program, and all other

\footnotetext{
*Dartmouth College, Hanover NH 03755, USA

${ }^{\dagger}$ Massachusetts Institute of Technology, Cambridge MA 02139, USA

${ }^{\ddagger}$ Dartmouth College, Hanover NH 03755, USA
} 
registers of $\pi$ are reset to $\perp$; however, the shared variables stored in the NVRAM are unaffected by a crash and retain their values. A crashed process $\pi$ eventually restarts, executing the program beginning from the instruction at the default location $\ell$, regardless of where in the program $\pi$ might have previously crashed.

When designing algorithms for this model, informally the goal is to ensure that when a crashed process restarts, it reconstructs the lost state by consulting the shared variables in NVRAM. To appreciate that this goal can be challenging, suppose that a process $\pi$ crashes when it is just about to perform an operation such as $r \leftarrow \operatorname{FAS}(X, 5)$, which fetches the value of the shared variable $X$ into $\pi$ 's register $r$ and then stores 5 in $X$. If a different process $\pi^{\prime}$ performs $\operatorname{FAS}(X, 10)$ and then $\pi$ restarts, $\pi$ cannot distinguish whether it crashed immediately before or immediately after executing its FAS instruction.

\subsection{The Recoverable Mutual Exclusion (RME) problem}

In the Recoverable Mutual Exclusion (RME) problem, there are $n$ asynchronous processes, where each process repeatedly cycles through four sections of code-Remainder, Try, Critical, and Exit sections. An algorithm (for RME) specifies the code for the Try and Exit sections of each process. Any process can execute a normal step or a crash step at any time. In a normal step of a process $\pi, \pi$ executes the instruction pointed by its program counter $P C_{\pi}$. We assume that if $\pi$ executes a normal step when in Remainder, $\pi$ moves to Try; and if $\pi$ executes a normal step when in CS, $\pi$ moves to Exit. A crash step models the crash of a process and can occur regardless of which section of code the process is in. A crash step of $\pi$ sets $\pi$ 's program counter to point to its Remainder section and sets all other registers of $\pi$ to $\perp$.

A run of an algorithm is an infinite sequence of steps. We assume every run satisfies the following conditions: (i) if a process is in Try, Critical, or Exit sections, it later executes a (normal or crash) step, and (ii) if a process enters Remainder because of a crash step, it later executes a (normal or crash) step.

An algorithm solves the RME problem if all of the following conditions are met in every run of the algorithm (Conditions (1), (3), (4) are from Golab and Ramaraju [7, and (2) and (5) are two additional natural conditions from Jayanti and Joshi [9]):

1. Mutual Exclusion: At most one process is in the CS at any point.

2. Wait-Free Exit: There is a bound $b$ such that, if a process $\pi$ is in the Exit section, and executes steps without crashing, $\pi$ completes the Exit section in at most $b$ of its steps.

3. Starvation Freedom: If the total number of crashes in the run is finite and a process is in the Try section and does not subsequently crash, it later enters the CS.

4. Critical Section Reentry (CSR) [7]: If a process $\pi$ crashes while in the CS, then no other process enters the CS during the interval from $\pi$ 's crash to the point in the run when $\pi$ next reenters the $C S$.

5. Wait-Free Critical Section Reentry (Wait-Free CSR) [9]: Given that the CSR property above mandates that after a process $\pi$ 's crash in the CS no other process may enter the CS until $\pi$ reenters the CS, it makes sense to insist that no process should be able to obstruct $\pi$ from reentering the CS. Specifically:

There is a bound b such that, if a process crashes while in the CS, it reenters the CS before completing $b$ consecutive steps without crashing.

(As observed in 9], Wait-Free CSR, together with Mutual Exclusion, implies CSR.) 


\subsection{Passage Complexity}

In a $\mathrm{CC}$ machine each process has a cache. A read operation by a process $\pi$ on a shared variable $X$ fetches a copy of $X$ from shared memory to $\pi$ 's cache, if a copy is not already present. Any non-read operation on $X$ by any process invalidates copies of $X$ at all caches. An operation on $X$ by $\pi$ counts as a remote memory reference (RMR) if either the operation is not a read or $X$ 's copy is not in $\pi$ 's cache. When a process crashes, we assume that its cache contents are lost. In a DSM machine, instead of caches, shared memory is partitioned, with one partition residing at each process, and each shared variable resides in exactly one partition. Any operation (read or non-read) by a process on a shared variable $X$ is counted as an RMR if $X$ is not in $\pi$ 's partition.

A passage of a process $\pi$ in a run starts when $\pi$ enters Try (from Remainder) and ends at the earliest later time when $\pi$ returns to Remainder (either because $\pi$ crashes or because $\pi$ completes Exit and moves back to Remainder).

A super-passage of a process $\pi$ in a run starts when $\pi$ either enters Try for the first time in the run or when $\pi$ enters Try for the first time after the previous super-passage has ended, and it ends when $\pi$ returns to Remainder by completing the Exit section.

The passage complexity (respectively, super-passage complexity) of an RME algorithm is the worst-case number of RMRs that a process incurs in a passage (respectively, in a super-passage).

\subsection{Our contribution}

The passage complexity of an RME algorithm can, in general, depend on $n$, the maximum number of processes the algorithm is designed for. The ideal of course would be to design an algorithm whose complexity is independent of $n$, but is this ideal achievable? It is well known that, for the traditional mutual exclusion problem, the answer is yes: MCS and many other algorithms that use FAS and CAS instructions have $O(1)$ passage complexity [3] 4] 11]. For the RME problem too, algorithms of $O(1)$ passage complexity are possible, but they use esoteric instructions not supported on real machines, such as Fetch-And-Store-And-Store (FASAS) and Double Word CAS, which manipulate two shared variables in a single atomic action [5] [8]. The real question, however, is how well can we solve RME using only operations supported by real machines.

With their tournament based algorithm, Golab and Ramaraju showed that $O(\log n)$ passage complexity is possible using only read and write operations [7]. In fact, in light of Attiya et al's lower bound result [2], this logarithmic bound is the best that one can achieve even with the additional support of comparison-based operations such as CAS. However in PODC '17, by using FAS along with CAS, Golab and Hendler [5] succeeded in breaching this logarithmic barrier for

CC machines: their algorithm has $O\left(\frac{\log n}{\log \log n}\right)$ passage complexity for CC machines, but unbounded passage complexity for DSM machines. In this paper we close this gap with the design of an algorithm that achieves the same sub-logarithmic complexity bound as theirs for both $\mathrm{CC}$ and DSM machines. Some additional advantages of our algorithm over Golab and Hendler's are:

1. Our algorithm satisfies the Wait-Free Exit property.

2. On a CC machine, Golab and Hendler's algorithm requires a cache of $\Theta(n)$ words at each process, but our algorithm needs a cache of only $O(1)$ words. (We explain the reason in the next subsection.)

3. Our algorithm needs only the FAS instruction (whereas Golab and Hendler's needs both FAS and CAS). 
4. Our algorithm eliminates the race conditions present in Golab and Hendler's algorithm that cause processes to starve ${ }^{1}$ (We describe these issues in detail in Appendix A.)

\subsection{Comparison to Golab and Hendler [5]: Similarities and differences}

Golab and Hendler [5] derived their sublogarithmic RME algorithm in the following two steps, of which the first step is the intellectual workhorse:

- The first step is the design of an RME algorithm, henceforth referred to as GH, of $O(n)$ passage complexity and $O(1+f n)$ super-passage complexity, where $f$ is the number of times that a process crashes in the super-passage. The exciting implication of this result is that, in the common case where a process does not fail in a super-passage, the process incurs only $O(1)$ RMRs in the super-passage.

- The second step is the design of an RME algorithm where the $n$ processes compete by working their way up on a tournament tree. This tree has $n$ leaves and each of the tree nodes is implemented by an instance of GH in which $\log n / \log \log n$ processes compete. (thus, the degree of each node is $\log n / \log \log n$, which makes the tree's height $O(\log n / \log \log n))$. The resulting algorithm has the desired $O\left(\frac{\log n}{\log \log n}\right)$ passage complexity and $O\left((1+f) \frac{\log n}{\log \log n}\right)$ super-passage complexity.

The GH algorithm is designed by converting the standard MCS algorithm [11] into a recoverable algorithm. As we now explain, this conversion is challenging because MCS uses the FAS instruction to insert a new node at the end of a queue. The queue has one node for each process waiting to enter the CS, and a shared variable TAIL points to the last node in the queue. When a process $\pi$ enters the Try section, it inserts its node $x$ into the queue by performing FAS(TAIL, $x$ ). The FAS instruction stores the pointer to $x$ in TAIL and returns TAIL's previous value prev to $\pi$ in a single atomic action. The value in prev is vital because it points to $x$ 's predecessor in the queue. Suppose that $\pi$ now crashes, thereby losing the prev pointer. Further suppose that a few more processes enter the Try section and insert their nodes behind $\pi$ 's node $x$. If $\pi$ now restarts, it cannot distinguish whether it crashed just before performing the FAS instruction or just after performing it. In the former case, $\pi$ will have to perform FAS to insert its node, but in the latter case it would be disastrous for $\pi$ to perform FAS since $x$ was already inserted into the queue. Yet, there appears no easy way for $\pi$ to distinguish which of the scenarios it is in. Notice further that, like $\pi$, many other processes might have failed just before or after their FAS, causing the queue to be disconnected into several segments. All of these failed processes, upon restarting, have to go through the contents of the shared memory to recognize whether they are in the queue or not and, if they are in, piece together their fragment with other fragments without introducing circularity or other blemishes in the queue. Since concurrent "repairing" by multiple recovering processes can lead to races, Golab and Hendler make the recovering processes go through an RME algorithm RLOCK so that at most one recovering process is doing the repair at any time. This RLOCK does not have to be too efficient since it is executed by only a failing process, which can afford to perform $O(n)$ RMRs. Thus, this RLOCK can be implemented using one of the known RME algorithms. However, while a process is trying to repair, correct processes can be constantly changing the queue, thereby making the repair task even more challenging.

The broad outline of our algorithm is the same as what we have described above for GH, but our algorithm differs substantially in important technical details, as we explain below.

\footnotetext{
${ }^{1}$ We communicated the issues described in Appendix A] with the authors of [5] who acknowledged the bugs and after a few weeks informed us that they were able to fix them.
} 
- In GH a recovering process raises a FAIL flag only after confirming that there is evidence that its FAS was successful. This check causes GH to deadlock (see Scenario 1 in Appendix A). Our algorithm eliminates this check.

- Since the shared memory can be constantly changing while a repairing process $\pi$ is scanning the memory to compute the disjointed fragments (so as to connect $\pi$ 's fragment to another fragment), the precise order in which the memory contents are scanned can be crucial to algorithm's correctness. In fact, we found a race condition in GH that can lead to segments being incorrectly pieced together: two different nodes can end up with the same predecessor, leading to all processes starving from some point on (see Scenario 2 in Appendix A).

- When a repairing process explores from each node $x$, GH does a "deep" exploration, meaning that the process visits $x$ 's predecessor $x_{1}, x_{1}$ 's predecessor $x_{2}, x_{2}$ 's predecessor $x_{3}$, and so on until the chain is exhausted. Our algorithm instead does a shallow exploration: it simply visits $x$ 's predecessor and stops there. The deep exploration of GH from each of the $n$ nodes leads to $O\left(n^{2}\right)$ local computation steps per passage and requires each process to have a large cache of $O(n)$ words in order to ensure the desired $O(n)$ passage-RMR-complexity. With our shallow exploration, we reduce the number of local steps per passage to $O(n)$ and the RMR complexity of $O(n)$ is achieved with a cache size of only $O(1)$ words.

- How an exiting process hands off the ownership of CS to the next waiting process is done differently in our algorithm so as to ensure a wait-free Exit section and eliminate the need for the CAS instruction.

\subsection{Related research}

Beyond the works that we discussed above, Golab and Hendler [6] presented an algorithm at last year's PODC that has the ideal $O(1)$ passage complexity, but this result applies to a different model of system-wide crashes, where a crash means that all processes in the system simultaneously crash. Ramaraju [12] and Jayanti and Joshi [9] design RME algorithms that also satisfy the FCFS property [10]. These algorithms have $O(n)$ and $O(\log n)$ passage complexity, respectively. Attiya, Ben-Baruch, and Hendler present linearizable implementations of recoverable objects [1].

\section{A Signal Object}

Our main algorithm, presented in the next section, relies on a "Signal" object, which we specify and implement in this section. The Signal object is specified in Figure 1, which includes a description of two procedures - set and wait - through which the object is accessed.

$X$. State $\in\{1,0\}$, initially 0 .

- $\quad X . \operatorname{set}()$ sets $X$.State to 1.

- $\quad X$.wait() returns when $X$.State is 1 .

Figure 1: Specification of a Signal object $X$.

\subsection{An implementation of Signal Object}

It is trivial to implement this object on a CC machine using a boolean variable BIT, initialized to 0 . To execute set(), a process writes 1 in BIT, and to execute wait(), a process simply loops until 
BIT has 1. With this implementation, both operations incur just $O(1)$ RMRs on a CC machine. Realizing $O(1)$ RMR complexity on a DSM machine is less trivial, especially because the identity of the process executing wait() is unknown to the process executing set(). Figure 2 describes our DSM implementation $\mathcal{X}$ of a Signal object $X$, which assumes that no two processes execute the wait() operation concurrently on the Signal object. Our implementation provides two procedures: $\mathcal{X}$.set () and $\mathcal{X}$.wait(). Process $\pi$ executes $\mathcal{X}$.set () to perform $X$.set() and $\mathcal{X}$.wait() to perform $X$.wait(). Our implementation ensures that a call to $\mathcal{X}$.set () and $\mathcal{X}$.wait() incur only $O(1)$ RMR.

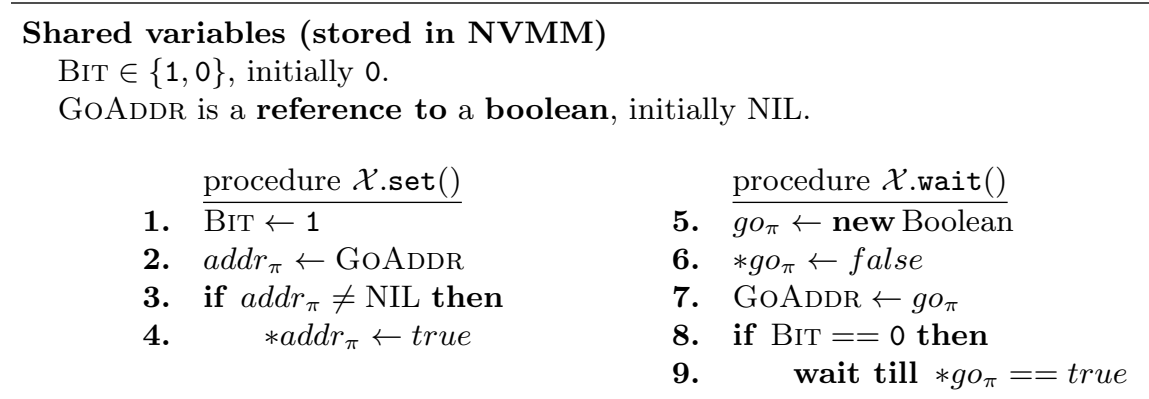

Figure 2: Implementation of a Signal object specified in Figure 1. Code shown for a process $\pi$.

When $\pi$ invokes $\mathcal{X}$.set (), at Line 1 it records for future $\mathcal{X}$.wait() calls that $X$.STATE $=1$, hence those calls can return without waiting. Thereafter, $\pi$ finds out if any process is already waiting for $X$.StATE to be set to 1 . It does so by checking if any waiting process has supplied the address of its own local-spin variable to $\pi$ on which it is waiting (Lines 2,3 . If $\pi$ finds that a process is waiting (i.e., $a d d r_{\pi} \neq \mathrm{NIL}$ ), then it writes true into that process's spin-variable to wake it up from the wait loop (Line 4 ).

When a process $\pi^{\prime}$ invokes $\mathcal{X}$.wait () , at Line 5 it creates a new local-spin variable that it hosts in its own memory partition (Line 5). It initializes that variable for waiting (Line 6) and notifies the object about its address (Line 7) so that the caller of $\mathcal{X}$.wait () can wake $\pi^{\prime}$ up as described above. Then $\pi^{\prime}$ checks if Bit $==1$ (Line 8), in which case $X$. STATE $=1$ already and $\pi^{\prime}$ can return without waiting. Otherwise, $\pi^{\prime}$ waits for $* g o_{\pi^{\prime}}$ to turn true (Line 9 ).

Theorem 1. $\mathcal{X}$.set () and $\mathcal{X}$.wait () described in Figure 2 implement a Signal object $\mathcal{X}$ (specified in Figure 1). Specifically, the implementation satisfies the following properties provided no two executions of $\mathcal{X}$.wait () are concurrent: (i) $\mathcal{X}$.set () is linearizable, i.e., there is a point in each execution of $\mathcal{X}$.set () when it appears to atomically set $\mathcal{X}$.STATE to 1 , (ii) When $\mathcal{X}$.wait () returns, $\mathcal{X}$.State is 1, (iii) A process completes $\mathcal{X}$.set() in a bounded number of its own steps, (iv) Once $\mathcal{X}$.State becomes 1 , any execution of $\mathcal{X}$.wait () by a process $\pi$ completes in a bounded number of $\pi$ 's steps, (v) $\mathcal{X} . \operatorname{set}()$ and $\mathcal{X}$.wait () incur $O(1) R M R$ on each execution.

\section{The Algorithm}

Our RME algorithm for $k$ ports is presented in Figures 3,4. We assume that all shared variables are stored in non-volatile main memory, and process local variables (subscripted by $\pi$ ) are stored in respective processor registers. We assume that if a process uses a particular port during its superpassage in a run, then no other process will use the same port during that super-passage. The process decides the port it will use inside the Remainder section itself. Therefore, the algorithm presented in Figures 34 is designed for use by a process $\pi$ on port $p$. 


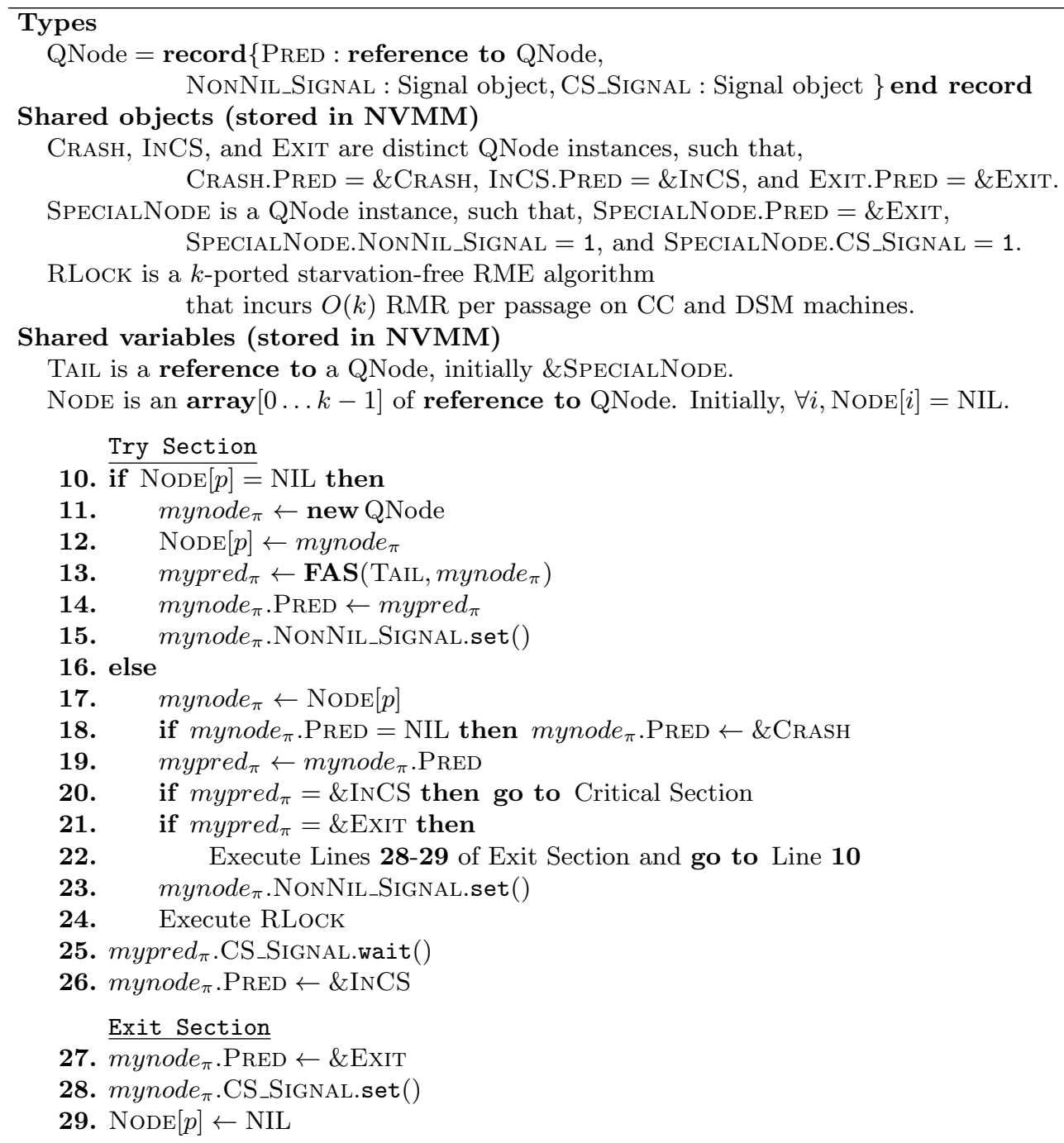

Figure 3: $k$-ported $n$-process RME algorithm for CC and DSM machines. Code shown for a process $\pi$ that uses port $p \in\{0, \ldots, k-1\}$. (Code continued in Figure 4.)

\subsection{Informal description}

The symbol \& is the usual "address of" operator, prefixed to a shared object to obtain the address of that shared object. The symbol "." (dot) dereferences a pointer and accesses a field from the record pointed to by that pointer. When invoked on a path $\sigma$ in a graph, the functions $\operatorname{start}(\sigma)$ and end $(\sigma)$ return the start and end vertices of the path $\sigma$. We assume that a process $\pi$ is in the Remainder section when $P C_{\pi}=\mathbf{1 0}$ and is in the CS when $P C_{\pi}=\mathbf{2 7}$.

Our algorithm uses a queue structure as in the MCS lock [11] and QNode is the node type used in such a queue. We modify the node structure in the following way to suit our needs. The node of a process $\pi$ has, apart from a PRED pointer, two instances of a Signal object: CS_SignAL and NonNil_Signal. $\pi$ 's successor process will use the CS_Signal instance from $\pi$ 's node to wait on $\pi$ before entering the CS. The NONNIL_Signal instance is used by any repairing process to wait till $\pi$ sets the PRED pointer of its node to a value other than NIL. Every node has a unique instance of these Signal objects. We ensure that the call to CS_SignAL.wait() happens from a single predecessor and the call to NonNiL_Signal.wait() is made in a mutually exclusive manner, 
Critical Section of RLOCK

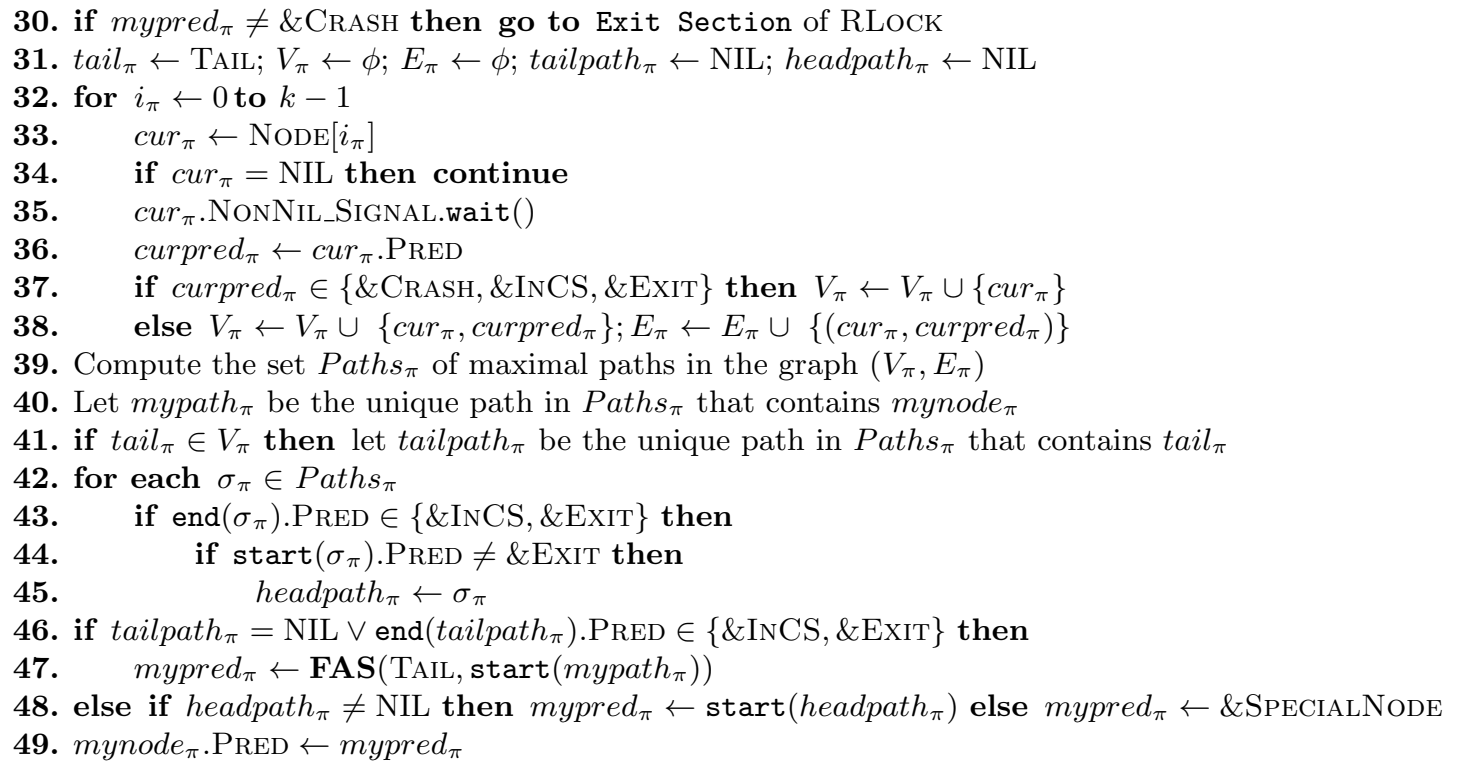

Figure 4: (Code continued from Figure 3.) $k$-ported $n$-process recoverable mutual exclusion algorithm for CC and DSM machines. Code shown for a process $\pi$ that uses port $p \in\{0, \ldots, k-1\}$. Vertex names in $V_{\pi}$ are node references, hence the "." symbol dereferences the address and accesses the members of the node. The functions $\operatorname{start}(\sigma)$ and end $(\sigma)$ used at Lines $43,44,46,48$ return the start and end vertices of the path $\sigma$.

thus ensuring that no two executions of wait () are concurrent on the same object instance. We also use an array of references to QNodes called NoDE[]. This is a reference to a QNode that is used by some process on port $p$ to complete a passage. In essence NODE $[p]$ binds a process $\pi$ to the port $p$ through the QNode $\pi$ uses for its passage.

We first describe how $\pi$ would execute the Try and Exit section in absence of a crash as follows, and then proceed to explain the algorithm if a crash is encountered anywhere. When a process $\pi$ wants to enter the CS through port $p$ from the Remainder section, it starts executing the Try section. At Line $\mathbf{1 0}$ it checks if any previous passage ended in a crash. If that is not the case, $\pi$ finds NoDE $[p]=$ NIL. It then executes Line 11 which allocates a new QNode for $\pi$ in the NVMM, such that the PRED pointer holds NIL, and the objects CS_Signal and NonNiL_Signal have STATE $=0$ (i.e., their initial values). At Line $\mathbf{1 2}$ the process stores a reference to this new node in $\operatorname{Node}[p]$ so that it can reuse this node in future in case of a crash. $\pi$ then links itself to the

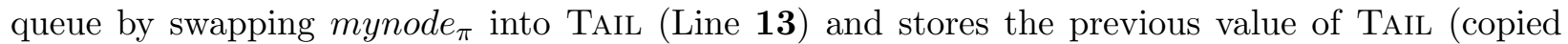

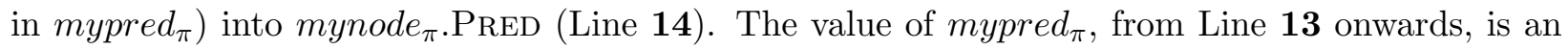
address of $\pi$ 's predecessor's node. At Line $\mathbf{1 5} \pi$ announces that it has completed inserting itself in the queue by setting mynode $e_{\pi}$.NonNIL_Signal to 1 (more later on why is this announcement important). $\pi$ then proceeds to Line 25 where it waits for mypred $_{\pi}$.CS_SignAL to become 1 . If the owner of the node pointed by mypred $\pi$ has already left the CS, then mypred $_{\pi}$. CS_SIGNAL is 1 ; otherwise, $\pi$ has to wait for a signal from its predecessor (see description of Signal object in previous section). Once $\pi$ comes out of the call to mypred $_{\pi}$.CS_SignaL.wait(), it makes a note in

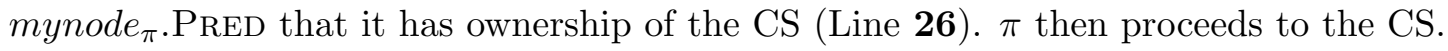

When $\pi$ completes the CS, it first makes a note to itself that it no longer needs the CS by writing \&EXIT in mynode $\pi$.PRED (Line 27). It then wakes up any successor process that might be 
waiting on $\pi$ to enter the CS (Line 28). $\pi$ then writes NIL into NoDE $[p]$ at Line 29, which signifies that the passage that used this node has completed.

When $\pi$ begins a passage after the previous passage ended in a crash, $\pi$ starts by checking Node $[p]$ at Line 10. If it has the value NIL, then $\pi$ crashed before it put itself in the queue, hence it treats the situation as if $\pi$ didn't crash in the previous passage and continues as described above. Otherwise, $\pi$ moves to Line $\mathbf{1 7}$ where it recovers the node it was using in the previous passage. If $\pi$ crashed while putting itself in the queue (i.e., right before executing Lines $\mathbf{1 3}$ or 14, it treats the crash as if it performed the FAS at Line $\mathbf{1 3}$ and crashed immediately. Hence, it makes

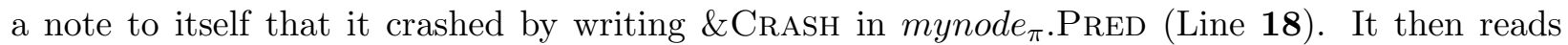
the value of mynode $_{\pi}$. PRED into mypred $_{\pi}$ (Line 19). At Line $20 \pi$ checks if it crashed while in the CS, in which case it moves to the CS. At Line 21 it checks if it already completed executing the CS, in which case recovery is done by executing Lines $\mathbf{2 8} \mathbf{2 9}$ and then re-executing Try from Line 10. If $\pi$ reaches Line 23 , it is clear that it crashed before entering the CS in the previous

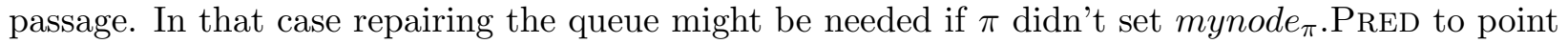
to a predecessor node. In any case, $\pi$ announces that mynode $_{\pi}$. PRED no longer has the value NIL setting mynode $\pi$.NonNiL_SignaL to $1 . \pi$ then goes on to capture RLOCK so that it gets exclusive access to repair the queue if it is broken at its node.

\section{High level view of repairing the queue after a crash}

Before diving into the code commentary of the CS of RLOCK, where $\pi$ repairs the queue broken at its end, we describe how the repairing happens at a high level. $\pi$ uses the RLock to repair the queue, if it crashed around the FAS operation (Lines 13 14 in the Try section. A crash by a process on Lines 13 14 can give rise to the following scenarios: (i) the queue is not affected by the crash (crash at Line $\mathbf{1 3}$ or at Line $\mathbf{1 4}$ but the queue was already broken), (ii) the queue is broken due to the crash (crash at Line 14). Therefore consider the following configuration 2 . Assume there is a node $x$ that was used by some process in its passage and the process has completed that passage succesfully so that $x$.PrED $=\&$ ExiT. Process $\pi_{1}, \pi_{3}$, and $\pi_{5}$ have crashed at Line 14. Process $\pi_{2}, \pi_{4}$, and $\pi_{6}$ are executing the procedure wait() at Line 25, such that, $\pi_{2}$ 's predecessor is $\pi_{1}$, $\pi_{4}$ 's predecessor is $\pi_{3}$, and $\pi_{6}$ 's predecessor is $\pi_{5}$. Process $\pi_{7}$ and $\pi_{8}$ have crashed at Line $\mathbf{1 3}$. We describe the repair by each of these crashed processes as follows.

Each of the crashed processes executes the RLOCK and waits for its turn to repair the queue in a mutually exclusive manner. Assume that the repair is performed by the processes in the order: $\left(\pi_{1}, \pi_{7}, \pi_{5}, \pi_{8}, \pi_{3}\right)$. When $\pi_{1}$ performs the repair, it first scans the NODE array and notices that the queue is broken at process $\pi_{4}$ and $\pi_{5}$ 's nodes (it notices that by reading \&CRASH in the PRED pointer of the process nodes). NODE array also gives an illusion to $\pi_{1}$ that queue is broken at $\pi_{7}$ and $\pi_{8}$ 's node although these processes didn't perform a FAS prior to their crash. $\pi_{1}$ also notices that no node has a predecessor node whose PRED pointer is set to \&INCS or \&ExIT, hence, no process is in the CS or is poised to enter it. Therefore $\pi_{1}$ sets its own node's predecessor to be Specialnode (from Figure 3, SpecialNode.Pred = \&Exit). Note, no other crashed process will set their own node's PRED pointer to point to SPECIALNODE simultaneously because repair operation is performed in a mutually exclusive manner by $\pi_{1}$. Also, the PRED pointer of each node has a non-NIL value (if not, then $\pi_{1}$ waits till it sees a non-NIL value before doing the actual repair). This way $\pi_{1}$ completes the repair operation on the queue and is now poised to enter the CS.

When $\pi_{7}$ (crashed at Line $\mathbf{1 3}$ ) performs the repair, it first scans the NODE array and notices that the queue is broken at process $\pi_{4}, \pi_{5}, \pi_{7}$, and $\pi_{8}$ 's nodes. Since it notices that no process

\footnotetext{
${ }^{2}$ Please refer to Figure 5 of Section $B$ in the Appendix for a visual illustration.
} 
points to $\pi_{2}$, it sets the PRED pointer of its own node to point to $\pi_{2}$ 's node. Thereby $\pi_{7}$ finishes the repair by placing itself in the queue, without ever performing the FAS, and gives up its control over RLOCK to return to the Try section.

When $\pi_{5}$ (crashed at Line $\mathbf{1 9}$ performs the repair, it follows an approach similar to that of $\pi_{7}$ 's. It sees that the queue is broken at process $\pi_{3}, \pi_{5}$, and $\pi_{8}$. It then notices that no process points to $\pi_{7}$ and therefore sets the PRED pointer of its own node to point to $\pi_{7}$ 's node. This way $\pi_{5}$ and $\pi_{6}$ are now attached to the queue in a way that there is a path from their node to a node containing the address \&ExiT. Also, TAIL points to $\pi_{6}$ 's node, so it appears as if the queue is unbroken if a traversal was done starting at the TAIL pointer.

Now that a traversal from TAIL would lead to a node used by a process that is in Critical section $\left(\pi_{1}\right.$ in this case), the queue is partially in place. In order to fix the remaining broken fragments the queue might need to be broken somehow to fit the remaining fragments. However, $\pi_{3}$ and $\pi_{8}$ can do the repair from here on without affecting the existing structure of the queue. $\pi_{8}$ can put itself in the queue by performing the FAS operation on the TAIL with its own node. Whereas $\pi_{3}$ first identifies the fragment its node is part of, and thereby all the nodes that are part of its fragment. It then performs a FAS one more time on TAIL with the last node in its own fragment (i.e., $\pi_{4}$ 's node) and sets the PRED pointer of its own node to the previous value of TAIL that is returned by the FAS (address of $\pi_{8}$ 's node). This ends the repair operation for $\pi_{3}$ and thereby the repair for all the process.

\section{Informal description of CS of RLOCK}

We proceed to give a description of the CS of RLOCK that does the above mentioned repair. At Line $30 \pi$ checks if it was already in the queue before its last crash (such a situation may occur either when $\pi$ crashes after executing the CS of RLOCK to completion but before executing the Exit section of RLOCK, or when $\pi$ crashes in the Try after performing Line 14. If so, it notices that there is no need for repair, hence, it goes to the Exit section of RLOCK. Otherwise, at Line $31 \pi$ reads the reference to the node pointed to by TAIL into the variable tail $_{\pi}$ and initializes other variables used during the repair procedure. Thereafter $\pi$ constructs a graph that models the queue structure. To this purpose, it reads each node pointed to by the NoDE array in order to construct the graph (Lines $\mathbf{3 2}$ 38). The graph is constructed as follows. First a cell from the Node array is read into cur $_{\pi}$ (i.e., NODE $\left[i_{\pi}\right]$ ) at Line 33 and checked if it is a node of some process (Line 34). If Node $\left[i_{\pi}\right]=\mathrm{NIL}, \pi$ moves on to the next cell in the array. Otherwise, at Line $\mathbf{3 5} \pi$ waits till $\operatorname{Node}\left[i_{\pi}\right]$.PRED assumes a non-NIL value (i.e., wait for the owner of that node to have executed either Line 15 or 23. Once cur $_{\pi}$ 's PRED pointer has a non-NIL value, that value is read into curpred $_{\pi}$ (Line 36). There are now two possibilities: (i) the PRED pointer points to one of \&CRASH, \&INCS, or \&EXIT, or (ii) the PRED pointer points to another node. The purpose of waiting for $\mathrm{cur}_{\pi}$.NONNIL_SIGNAL $=1$ is simple: we want to be sure which of the above two cases is true about cur $_{\pi}$. In the first case only cur $_{\pi}$ is added as a vertex to the graph (the name of that vertex is the value of cur $_{\pi}$ ). In the second case $c u r_{\pi}$ and curpred $_{\pi}$ are added as vertices and a directed edge $\left(\right.$ cur $_{\pi}$, curpred $\left._{\pi}\right)$ is added to the graph (we consider this as a simple graph, so repeated addition of a vertex counts as adding it once). This process is repeated until all cells from the NODE array are read. Once all the nodes are read from the cells of NODE array, including nodes not yet in the queue $\left(\pi_{7}\right.$ and $\pi_{8}$ in the above example), we have the graph $\left(V_{\pi}, E_{\pi}\right)$ that models the broken queue structure such that each maximal path in the graph models a broken queue fragment. Note, such a graph is a directed acyclic graph. At Line 39 set Paths $s_{\pi}$ of maximal paths in the graph $\left(V_{\pi}, E_{\pi}\right)$ is created and at Line 40 a path mypath $_{\pi}$ is picked from Paths $s_{\pi}$ such that mynode $e_{\pi}$ appears in it. At Line 41 a path tailpat $_{\pi}$ containing the node tail $_{\pi}$ is picked from Path $_{\pi}$ if tail $_{\pi}$ 
appears in the graph. In Lines 42,45 we try to find a path in the graph such that its start vertex belongs to a process that has not finished the critical section but a traversal on that path leads to a node holding one of the addresses \&INCS or \&ExIT (i.e., it leads to a node in or out of CS). If

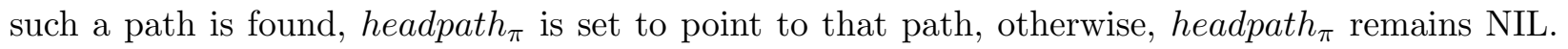
In Line 46 we first check if the queue is already partially repaired (e.g., if the repair was being performed by $\pi_{8}$ or $\pi_{3}$ in the example above). If so, at Line 47 the fragment containing mynode $e_{\pi}$ is inserted into the queue by performing a FAS on TAIL with the last node in that fragment (i.e.,

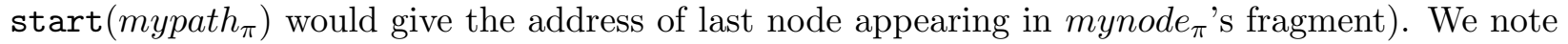
the previous value of TAIL into mypred $_{\pi}$ so that we can update mynode $e_{\pi}$.PRED later. Otherwise, $\pi$ needs to connect its own fragment to the queue. To this purpose it needs to be ensured that the queue is not broken at its head and some active process is poised to enter or is in the Critical section. Line 48 does this by checking if Lines 42,45 found a path in the graph such that its start vertex belongs to a process that has not finished the Critical section but a traversal on that path leads to a node out of CS (i.e., is headpath $h_{\pi} \neq \mathrm{NIL}$ ). If headpath $\neq$. NIL, then $\pi$ 's predecessor is set to be the start node on the path headpath $h_{\pi}\left(\pi_{7}, \pi_{5}\right.$ in the example above). Otherwise, the queue is broken at its head, therefore, at Line $48, \pi$ 's predecessor is set to be SpecialNode $\left(\pi_{1}\right.$ in example above). At Line 49, $\pi$ has the correct address to its predecessor node in mypred $_{\pi}$ (as noted in Lines 46 48 which is written into mynode ${ }_{\pi}$. PRED. This completes the CS of RLOCK and the repair of $\pi$ 's fragment. $\pi$ then proceeds back to Line 25 after completing the Exit section of RLOCK.

\subsection{Main theorem}

The correctness properties of the algorithm are captured in the following theorem.

Theorem 2. The algorithm in Figures 3.4 solves the RME problem for $k$ ports on CC and DSM machines and additionally satisfies the Wait-free Exit and Wait-free CSR properties. It has an $R M R$ complexity of $O(1)$ for a process that does not crash during its passage, and $O(f k)$ for a process that crashes $f$ times during its super-passage.

\section{3 $O((1+f) \log n / \log \log n)$ RMRs Algorithm}

To obtain a sub-logarithmic RMR complexity algorithm on both CC and DSM machines, we use the arbitration tree technique used by Golab and Hendler (described in Section 5 in [5]). Therefore, the following theorem follows from Theorem 2 .

Theorem 3. The arbitration tree algorithm solves the RME problem for $n$ processes on $C C$ and DSM machines and additionally satisfies the Wait-free Exit and Wait-free CSR properties. It has an RMR complexity of $O((1+f) \log n / \log \log n)$ per super-passage for a process that crashes $f$ times during its super-passage. 


\section{References}

[1] Attiya, H., Ben-Baruch, O., And Hendler, D. Nesting-Safe Recoverable Linearizability: Modular Constructions for Non-Volatile Memory. In Proceedings of the 2018 ACM Symposium on Principles of Distributed Computing (2018), ACM, pp. 7-16.

[2] Attiya, H., Hendler, D., And Woelfel, P. Tight RMR Lower Bounds for Mutual Exclusion and Other Problems. In Proc. of the Fortieth ACM Symposium on Theory of Computing (New York, NY, USA, 2008), STOC '08, ACM, pp. 217-226.

[3] Craig, T. S. Building FIFO and Priority-Queuing Spin Locks from Atomic Swap. Tech. Rep. TR-93-02-02, Department of Computer Science, University of Washington, February 1993.

[4] Dvir, R., And Taubenfeld, G. Mutual exclusion algorithms with constant RMR complexity and wait-free exit code. In Proceedings of The 21st International Conference on Principles of Distributed Systems (2017), OPODIS 2017.

[5] Golab, W., and Hendler, D. Recoverable mutual exclusion in sub-logarithmic time. In Proceedings of the ACM Symposium on Principles of Distributed Computing (New York, NY, USA, 2017), PODC '17, ACM, pp. 211-220.

[6] Golab, W., and Hendler, D. Recoverable Mutual Exclusion Under System-Wide Failures. In Proceedings of the 2018 ACM Symposium on Principles of Distributed Computing (New York, NY, USA, 2018), PODC '18, ACM, pp. 17-26.

[7] Golab, W., and Ramaraju, A. Recoverable Mutual Exclusion: [Extended Abstract]. In Proceedings of the 2016 ACM Symposium on Principles of Distributed Computing (New York, NY, USA, 2016), PODC '16, ACM, pp. 65-74.

[8] Jayanti, P., Jayanti, S., And Joshi, A. Optimal Recoverable Mutual Exclusion using only FASAS. In The 6th Edition of The International Conference on Networked Systems (2018), NETYS 2018.

[9] Jayanti, P., and Joshi, A. Recoverable FCFS mutual exclusion with wait-free recovery. In 31st International Symposium on Distributed Computing (2017), DISC 2017, pp. 30:1-30:15.

[10] Lamport, L. A New Solution of Dijkstra's Concurrent Programming Problem. Commun. ACM 17, 8 (Aug. 1974), 453-455.

[11] Mellor-Crummey, J. M., and Scott, M. L. Algorithms for Scalable Synchronization on Shared-memory Multiprocessors. ACM Trans. Comput. Syst. 9, 1 (Feb. 1991), 21-65.

[12] Ramaraju, A. RGLock: Recoverable mutual exclusion for non-volatile main memory systems. Master's thesis, University of Waterloo, 2015.

[13] Raoux, S., Burr, G. W., Breitwisch, M. J., Rettner, C. T., Chen, Y.-C., Shelby, R. M., Salinga, M., Krebs, D., Chen, S.-H., Lung, H.-L., Et Al. Phase-change random access memory: A scalable technology. IBM Journal of Research and Development 52, 4/5 (2008), 465.

[14] Strukov, D. B., Snider, G. S., Stewart, D. R., and Williams, R. S. The missing memristor found. nature 453, 7191 (2008), 80. 
[15] Tehrani, S., Slaughter, J. M., Deherrera, M., Engel, B. N., Rizzo, N. D., Salter, J., Durlam, M., Dave, R. W., Janesky, J., Butcher, B., et Al. Magnetoresistive random access memory using magnetic tunnel junctions. Proceedings of the IEEE 91, 5 (2003), $703-714$. 


\section{A Issues with Golab and Hendler's [5] Algorithm}

In this section we describe two issues with Golab and Hendler's FAS and CAS based algorithm. The Algorithm in question here appears in Figures 6, 7, 8 in [5] and we use the exact line numbers and variable names as they appear in the paper.

\section{A.1 Scenario 1: Process deadlock inside Recover}

The first issue with the GH algorithm is that processes deadlock waiting on each other inside the Recover section. This issue is described as below:

1. Process $P_{4}$ requests the lock by starting a fresh passage, goes to the CS, completes the Exit, and then goes back to Remainder.

2. Process $P_{2}$ starts a fresh passage, executes the code till (but not including) Line 26 and crashes.

3. Remainder section puts $P_{2}$ into Recover, $P_{2}$ starts executing IsLinkedTo(2) from Line 44 because mynode.nextStep $=26$ and mynode.prev $=\perp$ for $P_{2}$.

4. $P_{2}$ sleeps at Line 68 with $i=0$.

5. Process $P_{4}$ starts another passage, executes till (but not including) Line 26 and crashes.

6. Thereafter, $P_{4}$ goes to Recover, starts executing IsLinkedTo(4) from Line 44 because mynode.nextStep $=$ 26 and mynode.prev $=\perp$ for $P_{4}$.

7. $P_{2}$ starts executing procedure IsLinkedTo() where it left and executes several interations until $i=4$. Now it waits on lnodes[4].prev ( $P_{4}$ 's mynode) to become non- $\perp$.

8. $P_{4}$ starts executing procedure IsLinkedTo( $)$ where it left and executes several iterations until $i=2$ and now it waits on lnodes[2].prev ( $P_{2}$ 's mynode) to become non- $\perp$.

9. From now on no process including $P_{2}$ and $P_{4}$ ever crash. Therefore $P_{2}$ and $P_{4}$ are then waiting on each other and no one ever sets mynode.prev to a non- $\perp$ value. This results in violation of Starvation freedom property.

\section{A.2 Scenario 2: Starvation Freedom Violation}

The second issue with their algorithm is a process may starve even though it never crashed. The issue is as described below:

1. Process $P_{0}$ initiates a new passage, goes to CS, and no other process comes after it, so tail is pointing to $P_{0}$ 's node.

2. $P_{1}$ initiates a new passage, performs FAS on tail and goes behind $P_{0}$, and sets its own mynode.prev field to point to $P_{0}$ 's node.

3. $P_{2}$ initiates a new passage, performs FAS on tail and goes behind $P_{1}$, but crashes immediately, hence losing its local variable prev before setting its own mynode.prev field.

4. $P_{2}$ performs islinkedTo(2), which returns true because tail is pointing to $P_{2}$ 's mynode. 
5. $P_{3}$ initiates a new passage, performs FAS on tail and goes behind $P_{2}$, and sets its own mynode.prev field to point to $P_{2}$ 's node.

6. $P_{2}$ acquires $r$ Lock in order to recover from the crash, and performs iterations with $i=0,1,2,3$ of the for-loop on Line 76. At this point the relation $R$ maintained in the rlock contains $(0$, $1),(2,3),(3$, TAIL $)$.

7. $P_{4}$ initiates a new passage, performs FAS on tail and goes behind $P_{3}$, but loses its local variable prev before setting its own mynode.prev field.

8. $P_{5}$ initiates a new passage, performs FAS on tail and goes behind $P_{4}$, and sets its own mynode.prev field to point to $P_{4}$ 's node.

9. $P_{2}$ resumes and performs iterations with $i=4,5$ of the for-loop at Line 76 , adding $(4,5)$ to $R$.

At this point $\mathrm{R}=(0,1),(2,3),(3$, TAIL $),(4,5)$. Therefore, process 2 identifies

- $(0,1)$ as the non-failed fragment (segment 1$)$,

- $(4,5)$ as the middle segment (segment 2), and

- $(2,3),(3$, TAIL) as the tail segment (segment 3$)$.

10. On Line $93 P_{2}$ sets mynode.prev to point to $P_{5}$ 's node and tail still points to $P_{5}$ 's node.

11. $P_{6}$ initiates a new passage, performs FAS on tail and goes behind $P_{5}$, and sets its own mynode.prev field to point to $P_{5}$ 's node. Note, at this point, both $P_{2}$ and $P_{6}$ set their respective mynode.prev field to point to the $P_{5}$ 's node and tail points to $P_{6}$ 's node.

12. Thereafter $P_{6}$ executes the remaining lines of Try section setting $P_{5}$ 's mynode.next to point to its own node at Line 30, and then continues to busy-wait on Line 31 .

13. $P_{2}$ then comes out of the rlock, continues to Line 28 in Try, sets $P_{5}$ 's mynode.next to point to its own node at Line 30, and continues to busy-wait on Line 31.

14. Hereafter, assume that no process fails, we have that all the processes coming after $P_{6}$ including $P_{6}$ itself forever starve. This is because $P_{5}$ was supposed to wake $P_{6}$ up from the busy-wait, but it would wake up $P_{2}$ instead. $P_{2}$ never wakes any process up because it is not visible to any process. This violates Starvation Freedom.

\section{B Illustration for Repair}

Figure 5 illustrates the bird's eye view of queue repair performed by crashed processes. Refer to Section 3.1 for a detailed description. 


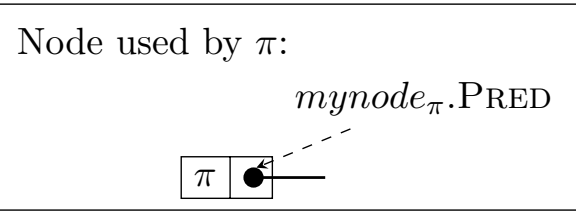

With $\pi_{1}, \pi_{3}, \pi_{5}, \pi_{7}$, and $\pi_{8}$ crashed, initial state of the queue $\left(\pi_{1}, \pi_{3}, \pi_{5}\right.$ crashed at Line $\mathbf{1 4}$ and $\pi_{7}, \pi_{8}$ crashed at Line $\mathbf{1 3}$ :

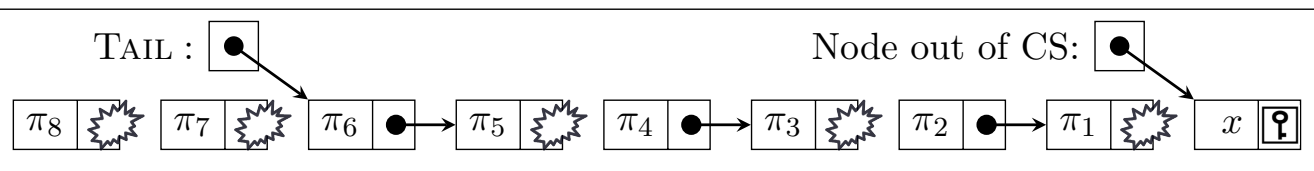

$\pi_{1}$ performs repair:

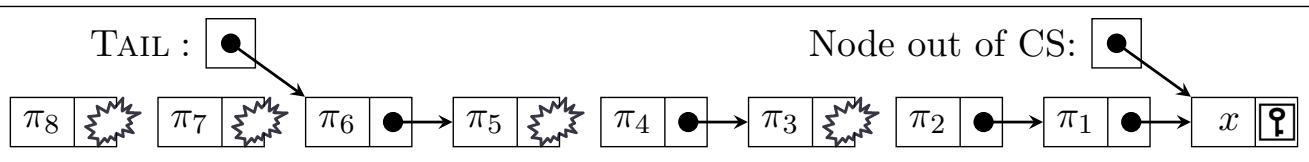

$\pi_{7}$ performs repair:

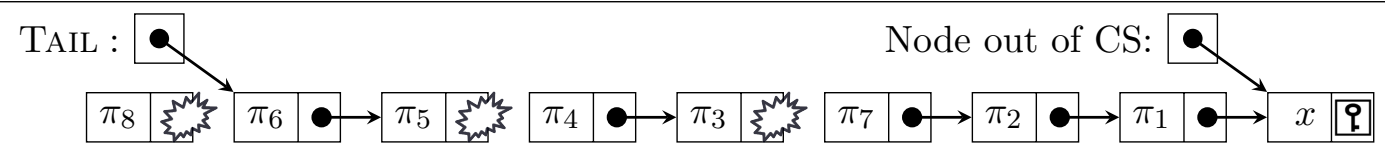

$\pi_{5}$ performs repair:

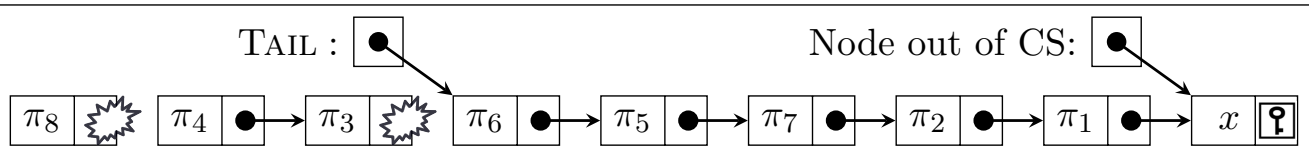

$\pi_{8}$ performs repair:

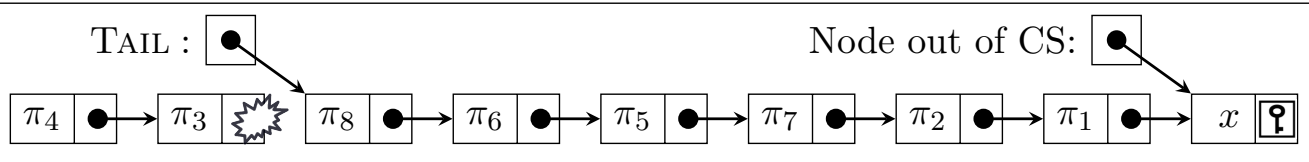

$\pi_{3}$ performs repair:

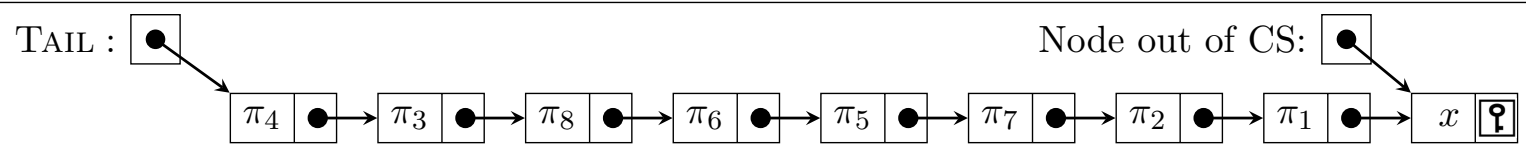

Figure 5: Queue states after repair is performed by different processes in a sequence. Explosion symbol in place of a PRED pointer on a node denotes the said process has crashed without updating the PRED pointer of its node. 


\section{Proof of correctness}

In this section we present a proof of correctness for the algorithm presented in Figures 344 . We prove the algorithm by giving an invariant for the algorithm and then proving correctness using the invariant. Figures 8,11 give the invariant satisfied by the algorithm. The proof that the algorithm satisfies the invariant is by induction and is presented in Appendix E.

We begin with some notation used in the proof and the invariant. A process may crash several times during its super-passage, at which point all its local variables get wiped out and the program counter is reset to $\mathbf{1 0}$ (i.e. first instruction of Try). In order to prove correctness we maintain a set of hidden variables that help us in the arguments of our proof. Following is the list of hidden variables for a process $\pi$ and the locations that the variables are updated in the algorithm:

$\widehat{\text { port }_{\pi}}$ : This variable stores the port number that $\pi$ uses to complete its super-passage. The Remainder section decides which port will be used by $\pi$ for the super-passage. When $\pi$ is not active in a super-passage, we assume that $\widehat{\text { port }_{\pi}}=$ NIL.

$\widehat{P C_{\pi}}$ : This variable takes line numbers as value according to the value of program counter, i.e., $P C_{\pi}$. Figures 6-77 show the annotated versions of our code from Figures 34 (annotations in $<>$ ) where we show the value that $\widehat{P C_{\pi}}$ takes at each line. We assume that the change in $\widehat{P C_{\pi}}$ happens atomically along with the execution of the line. $\widehat{P C_{\pi}}$ remains the same as before a line is executed for those lines in the figure that are not annotated (for example, Lines 10, 16, 21.

Try Section

10. if $\operatorname{NODE}[p]=$ NIL then

11. mynode $_{\pi} \leftarrow$ new QNode; $<\widehat{P C_{\pi}} \leftarrow \mathbf{1 2}>$

12. $\operatorname{NoDE}[p] \leftarrow$ mynode $_{\pi} ;<\widehat{P C_{\pi}} \leftarrow \mathbf{1 3}>$

13. mypred $_{\pi} \leftarrow$ FAS $\left(\right.$ TAIL, mynode $\left._{\pi}\right) ;<\widehat{P C_{\pi}} \leftarrow \mathbf{1 4}>$

14. mynode $_{\pi}$.PRED $\leftarrow$ mypred $_{\pi} ;<\widehat{P C_{\pi}} \leftarrow$ 15 $>$

15. mynode $_{\pi}$.NONNIL_SignAL.set ()$;<\widehat{P C_{\pi}} \leftarrow 25$

16. else

17. mynode $_{\pi} \leftarrow \operatorname{NODE}[p]$

18. if mynode $_{\pi}$.PRED $=$ NIL then mynode $_{\pi}$.PRED $\leftarrow$ \&CRASH

19. mypred $_{\pi} \leftarrow$ mynode $_{\pi}$.PRED

20. if mypred $_{\pi}=\&$ INCS then go to Critical Section

21. if mypred $_{\pi}=$ \&EXIT then

22. Execute Lines 28.29 of Exit Section and go to Line $10<\widehat{P C_{\pi}} \leftarrow \mathbf{1 1}>$

23. mynode $_{\pi}$.NONNIL_SIGNAL.set ()

24. Execute RLOCK

25. mypred $_{\pi}$.CS_SignAL.wait ()$;<\widehat{P C_{\pi}} \leftarrow \mathbf{2 6}>$

26. mynode $_{\pi}$.PRED $\leftarrow$ \&INCS; $<\widehat{P C_{\pi}} \leftarrow \mathbf{2 7}>$

Exit Section

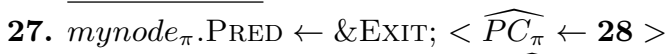

28. mynode $_{\pi}$.CS_SignAL.set ()$;<\widehat{P C_{\pi}} \leftarrow \mathbf{2 9}>$

29. $\mathrm{NODE}[p] \leftarrow \mathrm{NIL} ;<\widehat{P C_{\pi}} \leftarrow \mathbf{1 1}>$

Figure 6: Annotated version of code from Figure $3 . \widehat{\text { port }_{\pi}}=p$.

$\widehat{\operatorname{node}}_{\pi}$ : This variable is used to denote the QNode that $\pi$ is using in the current configuration for the current passage. Detailed description of the values that $\widehat{\operatorname{node}}_{\pi}$ takes appears in the Definitions section of Figure 8 . 
Critical Section of RLOCK

30. if mypred $_{\pi} \neq \&$ CRASH then

go to Exit Section of RLOCK;

$<\widehat{P C_{\pi}} \leftarrow 25>$

31. tail $_{\pi} \leftarrow$ TAIL $V_{\pi} \leftarrow \phi ; E_{\pi} \leftarrow \phi ;$ tailpath $_{\pi} \leftarrow$ NIL; headpath $h_{\pi} \leftarrow$ NIL

32. for $i_{\pi} \leftarrow 0$ to $k-1$

33. $\quad \operatorname{cur}_{\pi} \leftarrow \operatorname{NODE}\left[i_{\pi}\right]$

34. if $\mathrm{cur}_{\pi}=$ NIL then continue

35. $\quad \operatorname{cur}_{\pi}$.NONNIL_SignAL.wait ()

36. $\quad$ curpred $_{\pi} \leftarrow$ cur $_{\pi}$.PRED

37. if curpred $_{\pi} \in\{\& \mathrm{CRASH}, \& \mathrm{INCS}, \& \mathrm{EXIT}\}$ then $V_{\pi} \leftarrow V_{\pi} \cup\left\{\right.$ cur $\left._{\pi}\right\}$

38. else $V_{\pi} \leftarrow V_{\pi} \cup\left\{\right.$ cur $_{\pi}$, curpred $\left._{\pi}\right\} ; E_{\pi} \leftarrow E_{\pi} \cup\left\{\left(\right.\right.$ cur $_{\pi}$, curpred $\left.\left._{\pi}\right)\right\}$

39. Compute the set Paths $s_{\pi}$ of maximal paths in the graph $\left(V_{\pi}, E_{\pi}\right)$

40. Let mypath $_{\pi}$ be the unique path in Paths $s_{\pi}$ that contains mynode $_{\pi}$

41. if tail $_{\pi} \in V_{\pi}$ then let tailpath $_{\pi}$ be the unique path in Paths $s_{\pi}$ that contains tail $_{\pi}$

42. for each $\sigma_{\pi} \in$ Path $_{\pi}$

43. if end $\left(\sigma_{\pi}\right)$.PRED $\in\{\& \operatorname{INCS}, \& \operatorname{ExIT}\}$ then

44. if $\operatorname{start}\left(\sigma_{\pi}\right) \cdot \operatorname{PRED} \neq \&$ EXIT then

45. headpath $\leftarrow \sigma_{\pi}$

46. if tailpath $_{\pi}=\mathrm{NIL} \vee$ end $\left(\right.$ tailpath $\left._{\pi}\right)$.PRED $\in\{\& \mathrm{INCS}, \& \mathrm{EXIT}\}$ then

47. mypred $_{\pi} \leftarrow \operatorname{FAS}\left(\right.$ TAIL, start $\left(\right.$ mypath $\left.\left._{\pi}\right)\right) ;\left\langle\widehat{P C_{\pi}} \leftarrow 14>\right.$

48. else

if headpath $_{\pi} \neq$ NIL then mypred $_{\pi} \leftarrow \operatorname{start}\left(\right.$ headpath $\left._{\pi}\right)$ else mypred $_{\pi} \leftarrow \&$ SPECIALNODE $<\widehat{P C_{\pi}} \leftarrow \mathbf{1 4}>$

49. mynode $_{\pi} \cdot \mathrm{PRED} \leftarrow$ mypred $_{\pi} ;<\widehat{P C_{\pi}} \leftarrow \mathbf{2 5}>$

Figure 7: Annotated version of code from Figure $4 . \widehat{\text { port }_{\pi}}=p$.

We say that a process is in the CS if and only if $\widehat{P C_{\pi}}=27$. If $\pi$ is not active in a super-passage and hence in the Remainder section, $P C_{\pi}=\mathbf{1 0}, \widehat{P C_{\pi}}=\mathbf{1 5}$, and the values of the rest of the hidden variables are as defined above. We assume that initially all the local variables take arbitrary values.

Lemma 4 (Mutual Exclusion). At most one process is in the CS in every configuration of every run.

Proof. Suppose there are two processes $\pi_{i}$ and $\pi_{j}$ that are both in CS in a configuration $C$. Therefore, $\widehat{P C_{\pi_{i}}}=27$ and $\widehat{P C_{\pi_{j}}}=27$ in $C$. By definition of $\mathcal{Q}, \pi_{i} \in \mathcal{Q}$ and $\pi_{j} \in \mathcal{Q}$. Therefore, by Condition 19 of the invariant, one of the two processes is not $\pi_{1}$ in the ordering of processes in $\mathcal{Q}$. Without loss of generality, let $\pi_{i}=\pi_{1}$ and $\pi_{j}$ be a process coming later in the ordering. Therefore, by Condition $19(\mathrm{~d}) \mathrm{i}, \widehat{P C_{\pi_{j}}} \in\{\mathbf{1 5}, \mathbf{2 5}\}$, a contradiction.

Lemma 5 (Starvation Freedom). If the total number of crashes in the run is finite and a process is in the Try section and does not subsequently crash, it later enters the CS.

Proof. As noted in the statement of the claim, we assume that the total number of crashes in the run is finite.

A process $\pi$ using a port $p$ would not enter the CS during its passage if $P C_{\pi}$ is forever stuck at a certain line in the algorithm before entering the CS. Hence, in order to prove starvation freedom we have to argue that $P C_{\pi}$ advances to the next line for every step in the algorithm. An inspection of the Try section reveals that $\pi$ has procedure calls at Lines $\mathbf{1 5}, \mathbf{2 3}$, and $\mathbf{2 5}$, and inside the CS of RLock at Line $\mathbf{3 5}$. Since we require the RLOCK to be a recoverable starvation-free mutual exclusion lock, any process that executes Line $\mathbf{2 4}$ is guaranteed to eventually reach Line $\mathbf{3 0}$ of the Critical section of RLock (and hence reaches Line 35). Particularly, Golab and Ramaraju's 


\section{Assumptions:}

- Algorithm in Figures 3 assumes that every process uses a single port throughout its super-passage and no two processes execute a super-passage with the same port when their super-passages overlap. The Remainder section ensures that this assumption is always satisfied. Therefore, when a process continues execution after a crash, it uses the same port it chose at the start of the current super-passage. Hence, the Remainder section guarantees that the following condition is always met for active processes:

$$
\left.\forall \pi \in \Pi, \exists p \in \mathcal{P}, \widehat{\left(\text { ort }_{\pi}\right.}=p \wedge \forall p^{\prime} \in \mathcal{P}, p \neq p^{\prime}\right) \Rightarrow \widehat{\text { port }_{\pi}} \neq p^{\prime} .
$$

Definitions (Continued in Figure 9):

- $\mathcal{P}$ is a set of all ports.

- $\Pi$ is a set of all processes.

- $\mathcal{N}$ is a set containing the node SPECIALNode and any of the QNodes created by any process at Line $\mathbf{1 1}$ during the run so far.

- $\mathcal{N}^{\prime}=\{$ \&qnode $\mid$ qnode $\in \mathcal{N}\}$ is a set of node addresses from the nodes in $\mathcal{N}$.

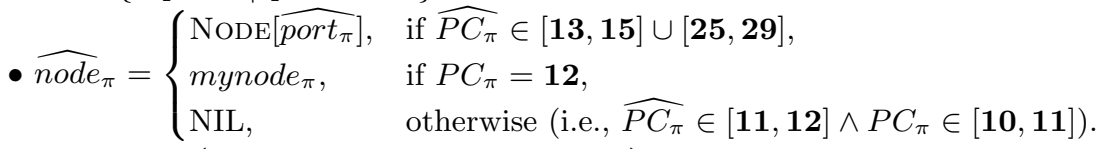

Conditions (Continued in Figures 9 10:

1. $\forall \pi \in \Pi,\left(\widehat{P C_{\pi}} \in\left\{[\mathbf{1 1},[12]\} \operatorname{NodE}\left[\widehat{\text { port }_{\pi}}\right]=\mathrm{NIL}\right) \wedge\left(\widehat{P C_{\pi}} \in\{\widehat{\mathbf{1 3}}, \mathbf{1 4}\} \Leftrightarrow \widehat{\text { node }}_{\pi} . \mathrm{PRED} \in\{\mathrm{NIL}, \& \mathrm{CRASH}\}\right)\right.$

$\wedge\left(\widehat{P C_{\pi}} \in\{\mathbf{1 5} \mathbf{2 5}, \mathbf{2 6}\} \Leftrightarrow \widehat{\text { node }}_{\pi} . \mathrm{PRED} \in \mathcal{N}^{\prime}\right) \wedge\left(\widehat{P C_{\pi}}=\mathbf{2 7} \Leftrightarrow \widehat{\text { node }}_{\pi} . \mathrm{PRED}=\right.$ \&INCS $)$

$\wedge\left(\widehat{P C_{\pi}} \in\{2 \mathbf{2 8}\} \Leftrightarrow \widehat{\operatorname{lode}}_{\pi}\right.$.PRED $=\&$ EXIT $)$

2. $\forall \pi \in \Pi,\left(P C_{\pi} \in \mathbf{1 3}, \mathbf{1 5} \cup\left[\mathbf{1 8}\left[\mathbf{2 9} \cup\left[\mathbf{3 0}, 48 \Rightarrow\right.\right.\right.\right.$ mynode $\left._{\pi}=\operatorname{NoDE}\left[\widehat{\text { ort }_{\pi}}\right]\right)$

$\wedge\left(P C_{\pi} \in\{\mathbf{1 5}\} \cup\left[\mathbf{2 0}, \mathbf{2 4} \cup\left[\mathbf{2 5}, \mathbf{2 6} \cup\left[\mathbf{3 0 , 4 8} \Rightarrow\right.\right.\right.\right.$ mypred $\left.\left._{\pi}={\text { NoDE }\left[\text { port }_{\pi}\right.}\right] . \mathrm{PRED}\right)$

$\wedge\left(\left(P C_{\pi} \in \mathbf{2 0}, \mathbf{2 4} \cup \mathbf{3 0}, \mathbf{4 8} \wedge \widehat{P C_{\pi}} \in\{\mathbf{1 3}, \mathbf{1 4}\}\right) \Rightarrow\right.$ mypred $\left._{\pi}=\& \mathrm{CRASH}\right)$

3. $\forall \pi \in \Pi, \mathrm{NoDE}\left[\widehat{\text { ort }_{\pi}}\right] \neq \mathrm{NIL} \Rightarrow\left(\mathrm{NODE}\left[\widehat{\text { port }_{\pi}}\right] \in \mathcal{N}^{\prime}\right.$

$$
\begin{aligned}
& \wedge\left(\left(\exists \pi^{\prime} \in \Pi, \pi \neq \pi^{\prime} \wedge \operatorname{NODE}\left[\widehat{\text { port }_{\pi}}\right] . \mathrm{PRED}=\operatorname{NoDE}\left[\widehat{\text { port }_{\pi^{\prime}}}\right]\right)\right. \\
& \vee\left(\operatorname{Node}\left[\widehat{\text { port }_{\pi}}\right] \cdot \operatorname{PrED} \in \mathcal{N}^{\prime} \wedge \mathrm{NODE}\left[\widehat{\text { port }_{\pi}}\right] \cdot \mathrm{PRED} \cdot \mathrm{PRED}=\& \text { ExIT }\right) \\
& \left.\vee \operatorname{Node}\left[\widehat{\text { port }_{\pi}}\right] . P R E D \in\{\text { NIL }, \& \text { CRASH }, \& \text { INCS }, \& E X I T\}\right) \\
& \wedge\left(\forall \pi^{\prime \prime} \in \Pi, \pi \neq \pi^{\prime \prime} \Rightarrow\right. \\
& \left(\left(\operatorname{NoDE}\left[\widehat{\text { port }_{\pi}}\right]=\operatorname{NODE}\left[\widehat{\text { port }_{\pi^{\prime \prime}}}\right] \Rightarrow \operatorname{NODE}\left[\widehat{\text { port }_{\pi}}\right]=\mathrm{NIL}\right)\right. \\
& \wedge\left(\operatorname{NODE}\left[\widehat{\text { port }_{\pi}}\right] \cdot \mathrm{PRED}=\mathrm{NODE}\left[\widehat{\text { port }_{\pi^{\prime \prime}}}\right] \cdot \mathrm{PRED} \Rightarrow\right. \\
& \text { Node } \left.\left.\left.\left[\widehat{\text { prt }_{\pi}}\right] . \text { PrEd } \in\{\text { NIL, \&CRASH, \&EXIT }\}\right)\right)\right) \text { ) }
\end{aligned}
$$

4. $\forall \pi, \pi^{\prime} \in \Pi,\left(\pi \neq \pi^{\prime} \Rightarrow\left(\widehat{\text { node }}_{\pi} \neq \widehat{\text { node }}_{\pi^{\prime}} \vee \widehat{\text { node }}_{\pi}=\widehat{\text { node }}_{\pi^{\prime}}=\mathrm{NIL}\right)\right)$

$$
\begin{aligned}
& \wedge\left(\left(\pi \neq \pi^{\prime} \wedge \widehat{\operatorname{node}}_{\pi} \neq \mathrm{NIL} \wedge \widehat{\operatorname{node}}_{\pi^{\prime}} \neq \mathrm{NIL}\right) \Rightarrow\right.
\end{aligned}
$$

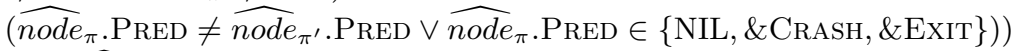

$$
\begin{aligned}
& \wedge\left(\exists b \in \mathbb{N},(1 \leq b \leq k \wedge \widehat{\text { node }}_{\pi} \underbrace{\text { PrEd.PrED } \cdots \text {.PRED }}_{b \text { times }} \in\{\text { NIL, \&CRASH, \&INCS, \&ExIT }\})\right)
\end{aligned}
$$

5. $\forall$ qnode $\in \mathcal{N}$, qnode.Pred $\in\{\mathrm{NIL}, \& \mathrm{CRASH}, \& \mathrm{INCS}, \& \mathrm{ExIT}\} \cup \mathcal{N}^{\prime}$

$$
\begin{aligned}
& \wedge\left(\left(\forall \pi \in \Pi, \widehat{\text { node }}_{\pi} \neq \text { \&qnode }\right) \Leftrightarrow\left(\forall p^{\prime} \in \mathcal{P}, \operatorname{NoDE}[p] \neq \text { qnode } \wedge \forall \pi^{\prime} \in \Pi, \text { mynode } \pi_{\pi^{\prime}} \neq \text { \&qnode }\right)\right) \\
& \wedge\left(\text { qnode.CS_SIGNAL }=1 \Rightarrow\left(\text { qnode.PRED }=\& \operatorname{ExIT} \wedge\left(\forall \pi \in \Pi, \widehat{\text { ode }_{\pi}}=\text { qnode } \Rightarrow \widehat{P C_{\pi}}=\mathbf{2 9}\right)\right)\right. \\
& \wedge \text { (qnode.NONNIL_SignAL }=1 \Rightarrow \\
& \text { (qnode.PRED } \left.\neq \mathrm{NIL} \wedge\left(\forall \pi \in \Pi, \widehat{\text { node }}_{\pi}=\text { qnode } \Rightarrow \widehat{P C_{\pi}} \in \text { 13, 15, 25, 29] }\right)\right) \text { ) } \\
& \wedge \text { (qnode.CS_SignAL }=0 \Rightarrow \text { qnode.PRED } \in\{\text { NIL, \&CRASH, \&INCS }\}) \\
& \wedge(\text { qnode.NonNiL_SigNAL }=0 \Rightarrow \text { qnode.PRED }=\text { NIL })
\end{aligned}
$$

Figure 8: Invariant for the $k$-ported recoverable mutual exclusion algorithm from Figures 3 ,4 (Continued in Figures 9.10.)

read-write based recoverable extension of Yang and Anderson's lock (see Section 3.2 in [7]) is one such lock that also guarantees a wait-free exit. Of these procedure calls, only the ones at Lines $\mathbf{2 5}$ and 35 concern us in the proof, since their implementation involves a wait loop. Therefore, if all the calls to wait are shown to complete, $\pi$ is guaranteed to enter the CS eventually.

We comment on a few other steps in the algorithm as follows before diving into the proof. The 


\section{Definitions (Continued from Figure 8):}

- For a QNode instance $\widehat{\operatorname{node}}_{\pi}$ used by a process $\pi \in \Pi$, fragment $\left(\widehat{\text { node }}_{\pi}\right)$ is a sequence of distinct QNode instances $\left(\widehat{\text { node }}_{\pi_{1}}, \widehat{\text { node }}_{\pi_{2}}, \ldots, \widehat{\operatorname{node}}_{\pi_{j}}\right)$ such that:

$-\forall i, \widehat{\operatorname{node}}_{\pi_{i}} \in \mathcal{N}$

- $\forall i \in[1, j-1], \widehat{\text { node }}_{\pi_{i+1}}$.PRED $=\widehat{\operatorname{node}}_{\pi_{i}}$ (e.g., $\widehat{\operatorname{node}}_{\pi_{2}} \cdot \operatorname{PRED}=\widehat{\operatorname{node}}_{\pi_{1}}$ ),

- $\widehat{\operatorname{node}}_{\pi_{1}}$.Pred $\in\{\mathrm{NIL}, \& \mathrm{CrASh}, \& \mathrm{INCS}, \& \mathrm{EXIT}\}$,

- $\forall q \in \mathcal{P}, \operatorname{NODE}[q] . \operatorname{PRED} \neq \widehat{\operatorname{node}}_{\pi_{j}}$,

$-\operatorname{head}\left(\operatorname{fragment}\left(\widehat{\operatorname{node}}_{\pi}\right)\right)=\widehat{\operatorname{node}}_{\pi_{1}}$ and tail $\left(\operatorname{fragment}\left(\widehat{\operatorname{node}}_{\pi}\right)\right)=\widehat{\operatorname{node}}_{\pi_{j}}$,

- $\mid$ fragment $\left(\widehat{\operatorname{node}}_{\pi}\right) \mid=j$.

For example, for the initial state of the queue in Figure $5 .\left(\pi_{1}, \pi_{2}\right),\left(\pi_{3}, \pi_{4}\right),\left(\pi_{5}, \pi_{6}\right),\left(\pi_{7}\right),\left(\pi_{8}\right)$ are distinct fragments. After $\pi_{3}$ performs repair in the illustration of Figure 5 the only fragment of the queue is: $\left(\pi_{1}, \pi_{2}, \pi_{7}, \pi_{5}, \pi_{6}, \pi_{8}, \pi_{3}, \pi_{4}\right)$. Note, in this example a node assumes the name of its process for brevity (i.e., $\pi_{1}$ should be read as $\widehat{\operatorname{node}}_{\pi_{1}}$ ). The set membership symbol $\in$ used on the sequence denotes membership of a node in the fragment. For example, $\pi_{2} \in \operatorname{fragment}\left(\pi_{1}\right)$ in both examples discussed above. Note, for simplicity we define fragment(NIL) $=$ NIL and $\mid$ fragment(NIL) $\mid=0$. Conditions of the invariant assert that the set of nodes in shared memory operated by the algorithm satisfy this definition of fragment.

- $\mathcal{Q}=\left\{\pi \in \Pi \mid\left(\widehat{P C_{\pi}} \in\{\mathbf{1 5}, \mathbf{2 5}, \mathbf{2 6}\} \wedge \operatorname{head}\left(f \operatorname{fragment}\left(\widehat{\text { ode }_{\pi}}\right)\right) . \operatorname{PRED} \in\{\& \operatorname{INCS}, \& \operatorname{EXIT}\}\right) \vee \widehat{P C_{\pi}}=\mathbf{2 7}\right\}$ is a set of queued processes.

Conditions (Continued from Figure 8):

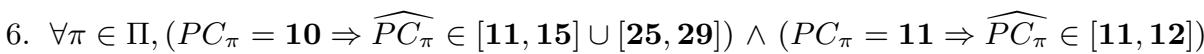

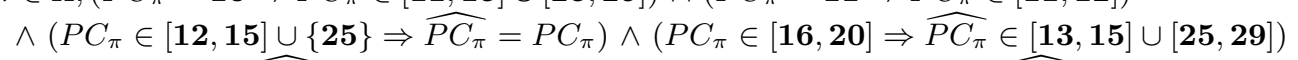

$\wedge\left(P C_{\pi}=\mathbf{2 1} \Rightarrow \widehat{P C_{\pi}} \in[\mathbf{1 3}, \mathbf{1 5} \cup \mathbf{2 5}, \mathbf{2 6}] \cup[\mathbf{2 8}, \mathbf{2 9}) \wedge\left(P C_{\pi}=\widehat{\mathbf{2 2}} \Rightarrow \widehat{P C_{\pi}} \in \underline{\mathbf{2 8}}, \mathbf{2 9}\right)\right.$

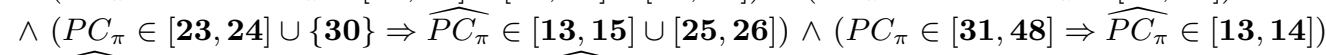

$\wedge\left(\widehat{P C_{\pi}}=\left[11 \Rightarrow P C_{\pi} \in\{10,[1]\}\right) \wedge\left(\widehat{P C_{\pi}}=[12] \Rightarrow P C_{\pi} \in[10] 12\right]\right)$

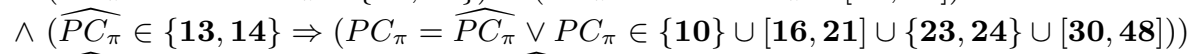

$\wedge\left(\widehat{P C_{\pi}} \in\{\mathbf{1 5}, \mathbf{2 5}, \mathbf{2 6}\} \Rightarrow\left(P C_{\pi}=\widehat{P C_{\pi}} \vee P C_{\pi} \in\{\mathbf{1 0}\} \cup[\mathbf{1 6}, \mathbf{2 1} \cup\{\mathbf{2 3}, \mathbf{2 4}\} \cup\{\mathbf{3 0}\})\right)\right.$

$\wedge\left(\widehat{P C_{\pi}}=\mathbf{2 7} \Rightarrow\left(P C_{\pi}=\widehat{P C_{\pi}} \vee P C_{\pi} \in\{[\mathbf{1 0}\} \cup[\mathbf{1 6}, \mathbf{2 0}])\right)\right.$

$\wedge\left(\widehat{P C_{\pi}} \in\{[\mathbf{2 8}]\right.$ 29] $\} \Rightarrow\left(P C_{\pi}=\widehat{P C_{\pi}} \vee P C_{\pi} \in\{[\mathbf{1 0}\} \cup[\mathbf{1 6},[\mathbf{2 2}))\right.$

7. $\forall \pi, \pi^{\prime} \in \Pi$, fragment $\left(\widehat{\text { node }}_{\pi}\right) \neq$ fragment $\left(\widehat{\text { node }}_{\pi^{\prime}}\right) \Rightarrow$ $\left(\forall \pi^{\prime \prime} \in \Pi,{\widehat{\text { node }_{\pi^{\prime \prime}}}}_{i \operatorname{fragment}}\left(\widehat{\operatorname{node}}_{\pi}\right) \Rightarrow \widehat{\operatorname{node}}_{\pi^{\prime \prime}} \notin \operatorname{fragment}\left(\widehat{\text { node }}_{\pi^{\prime}}\right)\right)$

$\wedge$ head $\left(\right.$ fragment $\left.\left(\widehat{\text { node }}_{\pi}\right)\right)$. PRED $=\& \operatorname{INCS} \Rightarrow\left(\left(\pi \neq \pi^{\prime} \wedge\right.\right.$ head $\left(\right.$ fragment $\left.\left(\widehat{\text { node }}_{\pi^{\prime}}\right)\right)$. PRED $=\&$ INCS $) \Rightarrow$ $\widehat{\text { node }}_{\pi^{\prime}} \in$ fragment $\left.\left(\widehat{\text { node }}_{\pi}\right)\right)$

$\wedge \operatorname{head}\left(\operatorname{fragment}\left(\widehat{\operatorname{node}}_{\pi}\right)\right) \cdot \operatorname{PRED}=\& \operatorname{ExIT} \Rightarrow\left(\widehat{P C_{\pi}} \in \underline{\mathbf{2 8}}, \mathbf{2 9} \vee\right.$

$\left(\pi \neq \pi^{\prime} \wedge\right.$ head $\left(\right.$ fragment $\left(\right.$ node $\left.\left._{\pi^{\prime}}\right)\right)$. PRED $\left.=\& \operatorname{ExIT} \wedge \widehat{P C_{\pi^{\prime}}} \notin \underline{\mathbf{2 8}}, \mathbf{2 9}\right) \Rightarrow$ $\left.\widehat{\operatorname{node}}_{\pi^{\prime}} \in \operatorname{fragment}\left(\widehat{\operatorname{node}}_{\pi}\right)\right)$

$\wedge\left(\mid\right.$ fragment $\left(\widehat{\operatorname{mode}}_{\pi}\right) \mid>1 \Rightarrow$

$$
\left.\left(\left(\text { node }_{\pi^{\prime}} \in \operatorname{fragment}\left(\widehat{\text { node }}_{\pi}\right) \wedge \widehat{\text { node }}_{\pi^{\prime}} \neq \operatorname{head}\left(\operatorname{fragment}\left(\widehat{\text { node }}_{\pi}\right)\right)\right) \Rightarrow \widehat{P_{\pi^{\prime}}} \in\{\mathbf{1 5}, \mathbf{2 5}\}\right)\right)
$$

8. $\forall \pi \in \Pi, P C_{\pi} \in\left\{\mathbf{1 2}[\mathbf{1 3}\} \Rightarrow\left(\right.\right.$ mynode $_{\pi} \in \mathcal{N}^{\prime} \wedge\left(\forall q \in \mathcal{P}, \operatorname{NodE}[q] \neq\right.$ mynode $_{\pi} \wedge \operatorname{NOdE}[q]$. Pred $\neq$ mynode $\left._{\pi}\right)$ $\wedge$ mynode $_{\pi}$. CS_SignaL $=0 \wedge$ mynode $_{\pi}$.NonNiL_SignaL $=0$

$\wedge$ mynode $_{\pi}=\operatorname{head}\left(\right.$ fragment $\left(\right.$ mynode $\left.\left._{\pi}\right)\right) \wedge \mid$ fragment mynode $\left._{\pi}\right) \mid=1$

$\wedge$ fragment $\left(\right.$ mynode $\left._{\pi}\right) \neq$ fragment $($ TAIL $) \wedge$ mynode $_{\pi}$ PRED $\left.=\mathrm{NIL}\right)$

Figure 9: (Continued from Figure 8) ) Invariant for the $k$-ported recoverable mutual exclusion algorithm from Figures 34 . (Continued in Figure 10.)

for loop at Line $\mathbf{3 2}$ executes for $k$ iterations, therefore, Lines $\mathbf{3 2} 3 \mathbf{3 8}$ execute a bounded number of times. Computing the set of maximal paths at Line $\mathbf{3 9}$ is a local computation step and has a bounded time algorithm, therefore, the step is executed a bounded number of times. The set $\mathrm{Path}_{\pi}$ is a finite set and finding the path mypath $_{\pi}$ at Line 40 is a local computation step which has 


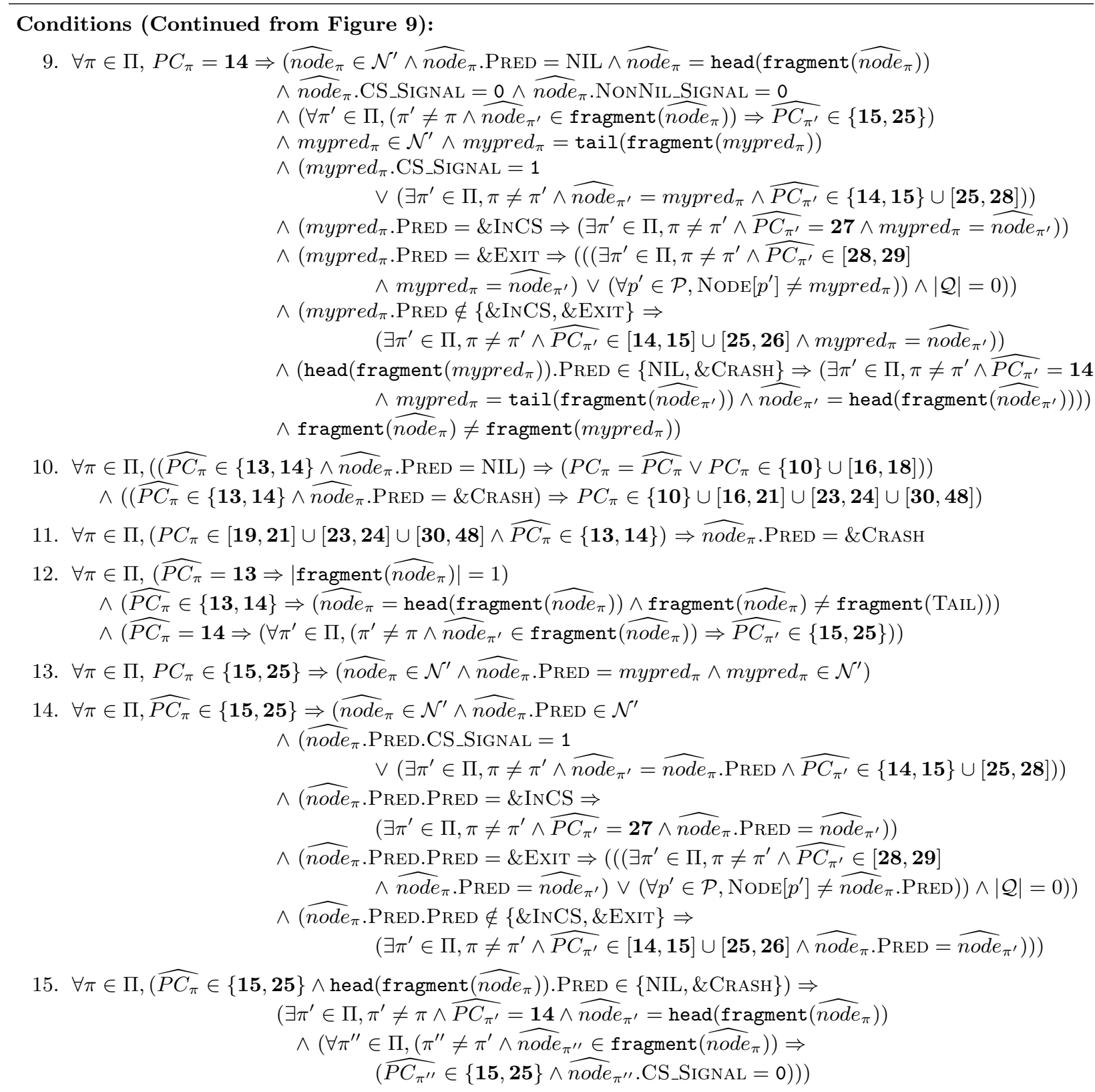

Figure 10: (Continued from Figure 9.) Invariant for the $k$-ported recoverable mutual exclusion algorithm from Figures 3.4. (Continued in Figure 11.)

a bounded time algorithm, therefore, the step is executed a bounded number of times. Similarly, Line 41 is a local computation step which has a bounded time algorithm, therefore, the step is executed a bounded number of times. As observed above, Path $s_{\pi}$ is a finite set, therefore the loop at Line 42 iterates a finite number of times. Hence, Lines 42,45 execute a bounded number of times. Note, since our algorithm has a wait-free exit (see Lemma 6), $\pi$ goes back to the Remainder section in a bounded number of normal steps once it finishes the CS. From the above it follows that $\pi$ executes wait loops inside the calls for wait only at Lines $\mathbf{2 5}$ and $\mathbf{3 5}$. Therefore, we consider these two cases where $\pi$ could potentially loop as follows and ensure that it eventually gets past these lines. 


\section{Conditions (Continued from Figure 10):}

16. TAIL $\in \mathcal{N}^{\prime} \wedge$ TAIL $=$ tail $($ fragment $($ TAIL $)) \wedge(\exists i \in[0, k-1]$, TAIL $=\operatorname{NodE}[i] \vee$ TAIL.PRED $=\&$ ExIT $)$

$\wedge$ (TAIL.CS_SIGNAL $=1$

$$
\vee\left(\exists \pi^{\prime} \in \Pi, \pi \neq \pi^{\prime} \wedge \widehat{\text { node }}_{\pi^{\prime}}=\text { TAIL } \wedge \widehat{P C_{\pi^{\prime}}} \in\{\mathbf{1 4}[\mathbf{1 5}\} \cup[\mathbf{2 5}, \mathbf{2 8}))\right.
$$

$\wedge\left(\right.$ TAIL.PRED $=\&$ INCS $\Rightarrow\left(\exists \pi^{\prime} \in \Pi, \pi \neq \pi^{\prime} \wedge \widehat{P C_{\pi^{\prime}}}=27 \wedge\right.$ TAIL $\left.\left.=\widehat{\text { node }_{\pi^{\prime}}}\right)\right)$

$\wedge\left(\right.$ TAIL.PrEd $=\&$ Exit $\Rightarrow\left(\left(\left(\exists \pi^{\prime} \in \Pi, \widehat{P C_{\pi^{\prime}}} \in \underline{\mathbf{2 8}}, \mathbf{2 9} \wedge\right.\right.\right.$ TAIL $\left.=\widehat{\text { node }}_{\pi^{\prime}}\right) \vee\left(\forall p^{\prime} \in \mathcal{P}, \operatorname{NodE}\left[p^{\prime}\right] \neq\right.$ TAIL $\left.)\right)$ $\wedge|\mathcal{Q}|=0))$

$\wedge\left(\right.$ TAIL.PRED $\notin\{\&$ InCS,$\&$ EXIT $\} \Rightarrow\left(\exists \pi^{\prime} \in \Pi, \widehat{P C_{\pi^{\prime}}} \in\left[\mathbf{1 4}\left[\mathbf{1 5} \cup\left[\mathbf{2 5}[\mathbf{2 6}] \wedge\right.\right.\right.\right.$ TAIL $\left.\left.\left.=\widehat{\text { node }}_{\pi^{\prime}}\right)\right)\right)$

$\wedge\left(\right.$ head $($ fragment $($ TAIL $))$. Pred $\in\{\mathrm{NIL}, \& \mathrm{CrASH}\} \Rightarrow\left(\exists \pi^{\prime} \in \Pi, \widehat{P C_{\pi^{\prime}}}=14\right.$ $\wedge$ TAIL $=\operatorname{tail}\left(\right.$ fragment $\left.\left(\widehat{\text { node }}_{\pi^{\prime}}\right)\right) \wedge \widehat{\text { node }}_{\pi^{\prime}}=\operatorname{head}\left(\right.$ fragment $\left.\left.\left.\left(\widehat{\text { node }}_{\pi^{\prime}}\right)\right)\right)\right)$

$\wedge\left(\left(\exists \pi \in \Pi, \widehat{P C_{\pi}} \in[\mathbf{1 4}] \mathbf{1 5} \cup[\mathbf{2 5}, \mathbf{2 9}) \Leftrightarrow\left(\exists \pi^{\prime} \in \Pi\right.\right.\right.$, TAIL $\left.=\widehat{\text { node }}_{\pi^{\prime}} \wedge \widehat{P C_{\pi^{\prime}}} \in[\mathbf{1 4}[\mathbf{1 5}] \cup[\mathbf{2 5}][\mathbf{2 9}])\right)$

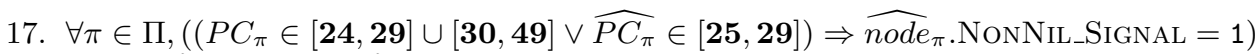

$\wedge\left(\widehat{P C_{\pi}}=2 \mathbf{2 9} \Rightarrow{\widehat{\text { node }_{\pi}}}_{\text {.CS_SignAL }}=1\right)$

18. $|\mathcal{Q}|=0 \Rightarrow\left(\left(\right.\right.$ TAIL.PRED $=\& \operatorname{EXIT} \vee \exists \pi \in \Pi,\left(\widehat{P C_{\pi}}=\widehat{14} \wedge\right.$ TAIL $=\operatorname{tail}\left(\right.$ fragment $\left.\left(\widehat{\text { node }}_{\pi}\right)\right)$

$$
\left.\left.\wedge \widehat{\text { node }}_{\pi}=\operatorname{head}\left(\text { fragment }\left(\widehat{\text { node }}_{\pi}\right)\right)\right)\right)
$$

$\wedge\left(\forall \pi^{\prime} \in \Pi, P C_{\pi^{\prime}} \in \mathbf{1 1} \mathbf{1 5} \cup\{\underline{\mathbf{2 5}}\} \cup[\mathbf{2 8}, \mathbf{2 9})\right)$

19. If $|\mathcal{Q}|=l>0$, then there is an order $\pi_{1}, \pi_{2}, \ldots, \pi_{l}$ of distinct processes in $Q$ such that:

(a) $\widehat{P C_{\pi_{1}}} \in\{\underline{\mathbf{1 5}}\} \cup \underline{\mathbf{2 5}}, \mathbf{2 7}$

(b) $\left(\exists \pi \in \Pi, \widehat{P C_{\pi}} \in \mathbf{2 8}, \mathbf{2 9} \wedge \widehat{\text { node }}_{\pi_{1}}\right.$.PRED $\left.=\widehat{\operatorname{node}}_{\pi}\right)$ $\vee\left(\widehat{\text { node }}_{\pi_{1}}\right.$.PRED $\left.\in \mathcal{N}^{\prime}-\left\{\widehat{\text { node }}_{\pi^{\prime}} \mid \pi^{\prime} \in \Pi \wedge \widehat{\text { node }}_{\pi^{\prime}} \neq \mathrm{NIL}\right\}\right)$

(c) $\widehat{P C_{\pi_{1}}} \in\{\underline{\mathbf{1 5}}, \mathbf{2 5}\} \Rightarrow\left(\widehat{\text { node }}_{\pi_{1}}\right.$.PrED.CS_SignaL $=1 \vee$ $\left(\exists \pi^{\prime} \in \Pi, \pi_{1} \neq \pi^{\prime} \wedge \widehat{\text { ode }}_{\pi^{\prime}}=\widehat{\text { node }}_{\pi_{1}}\right.$.PRED $\left.\wedge \widehat{P C_{\pi^{\prime}}}=\underline{\mathbf{2 8}}\right)$

(d) $\forall i \in[2, l]$ :

i. $\widehat{P C_{\pi_{i}}} \in\{\mathbf{1 5}, \mathbf{2 5}\}$

ii. $\widehat{\operatorname{node}}_{\pi_{i}} \cdot \mathrm{PRED}=\widehat{\operatorname{node}}_{\pi_{i-1}}$

Observation: $\widehat{\operatorname{node}}_{\pi_{i}} \in \operatorname{fragment}\left(\widehat{\operatorname{node}}_{\pi_{1}}\right)$.

(e) $\widehat{\operatorname{node}}_{\pi_{l}}=\operatorname{tail}\left(\right.$ fragment $\left.\left(\widehat{\text { node }}_{\pi_{1}}\right)\right)$

(f) $\widehat{\operatorname{node}}_{\pi_{1}}=\operatorname{head}\left(\right.$ fragment $\left.\left(\widehat{\text { node }}_{\pi_{1}}\right)\right) \vee \widehat{\text { node }}_{\pi_{1}}$.PRED.PRED $=\&$ ExIT

(g) $\forall \pi \in \Pi, \pi \neq \pi_{1} \Rightarrow \widehat{P C_{\pi}} \in \mathbf{1 1}, \mathbf{1 5} \cup\{\mathbf{2 5}\} \cup[\mathbf{2 8}, \mathbf{2 9}$

(h) $\forall \pi \in \Pi,\left(\pi \neq \pi_{1} \wedge \widehat{\operatorname{node}}_{\pi} \neq \mathrm{NIL} \wedge \widehat{\operatorname{node}}_{\pi}\right.$. Pred $\left.\in \mathcal{N}^{\prime}\right) \Rightarrow\left(\widehat{\text { node }}_{\pi}\right.$. Pred.CS_Signal $\left.=0\right)$

Observation: $\forall \pi \in \Pi, \pi \neq \pi_{1} \Rightarrow \widehat{P C_{\pi}} \neq \mathbf{2 7}$

Proof: If $\pi \in \mathcal{Q}$, then by Condition $\sqrt{19(\mathrm{~d}) \mathrm{i}} \widehat{P C_{\pi}} \neq \mathbf{2 7}$ If $\pi \notin \mathcal{Q}$, then, $\widehat{P C_{\pi}} \neq \mathbf{2 7}$ by definition of $\mathcal{Q}$.

Figure 11: (Continued from Figure 10.) Invariant for the $k$-ported recoverable mutual exclusion algorithm from Figures 3.4.

Case 1: $\pi$ completes the step at Line 35.

When $P C_{\pi}=$ 35, by Condition 30 , cur $_{\pi}$.NonNiL_Signal $=1$ or $\left(\exists \pi^{\prime} \in \Pi, \pi \neq \pi^{\prime} \wedge\right.$ cur $_{\pi}=\widehat{\text { node }}_{\pi^{\prime}} \wedge$ $\widehat{P C_{\pi^{\prime}}} \in[13,15)$. Suppose $c u r_{\pi}$. NONNIL_SignAL $=1$. $c u r_{\pi}$. NONNIL_Signal is an instance of the Signal object from Section 2.1, it follows that the call to $\mathrm{cur}_{\pi}$.NonNiL_SignaL.wait() on Line 35 returns in a wait-free manner. Therefore, $\pi$ completes the step at Line $\mathbf{3 5}$.

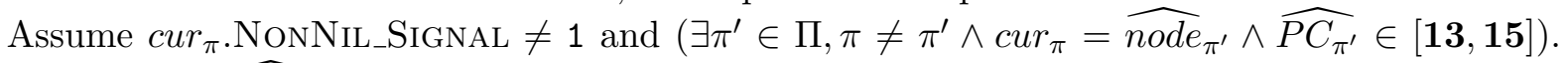
Suppose $P C_{\pi^{\prime}}=\widehat{P C_{\pi^{\prime}}}$ and there are no crash steps by $\pi^{\prime}$ before completing Line $\mathbf{1 5}$. In that case $\pi^{\prime}$ executes cur $_{\pi}$.NONNIL_Signal.set () to completion at Line $\mathbf{1 5}$ and sets cur $_{\pi}$.NONNIL_SignAL $=1$ in a wait-free manner. It follows that the call to cur $_{\pi}$.NonNIL_SignAL.wait() on Line 35 returns 
subsequently in a wait-free manner. Therefore, assume that $P C_{\pi^{\prime}} \neq \widehat{P C_{\pi^{\prime}}}$. By Conditions 6,17 and the fact that $c u r_{\pi}$.NonNiL_Signal $\neq 1, P C_{\pi^{\prime}} \in\{\mathbf{1 0}\} \cup[\mathbf{1 6}, 21] \cup\{\mathbf{2 3}\}$. Therefore, $\pi^{\prime}$ eventually executes cur $_{\pi}$.NONNIL_Signal.set () to completion at Line 23 and sets cur $_{\pi}$.NonNIL_SIGNAL $=$ 1 in a wait-free manner. It follows that the call to $c u r_{\pi}$.NonNiL_Signal.wait() on Line 35 returns subsequently in a wait-free manner. Note, in case of a crash by $\pi^{\prime}$ before executing cur $_{\pi}$.NONNIL_SignAL.set () to completion, $\pi^{\prime}$ starts at Line $\mathbf{1 0}$ and reaches Line $\mathbf{2 3}$. This is because $\widehat{P C_{\pi^{\prime}}} \in[13,15]$ implies Node $\left[\widehat{\text { port }}_{\pi^{\prime}}\right] \neq$ NIL and $\widehat{\text { node }}_{\pi^{\prime}}$.PrED $\notin\{\&$ INCS, \&ExIT $\}$. Therefore, the if conditions at Lines $\mathbf{1 0}, \mathbf{2 0}$, and $\mathbf{2 1}$ are not met and $\pi^{\prime}$ reaches Line $\mathbf{2 3}$. From the above it follows that $\pi$ completes the step at Line $\mathbf{3 5}$.

Case 2: $\pi$ completes the step at Line 25 .

In order to argue that $\pi$ completes the step at Line 25, we consider two cases. For the first case we have head(fragment $\left.\left(\widehat{\operatorname{node}}_{\pi}\right)\right)$.PRED $\in\{\& \mathrm{INCS}, \&$ ExIT $\}$ and the second occurs when head $\left(\right.$ fragment $\left.\left(\widehat{\operatorname{node}}_{\pi}\right)\right)$.PrEd $\in\{\mathrm{NIL}, \& \mathrm{CRASH}\}$. The first case occurs when $\pi \in \mathcal{Q}$ and the second occurs when $\pi \notin \mathcal{Q}$, both because of the value of head(fragment $\left.\left(\widehat{\operatorname{node}}_{\pi}\right)\right)$.PRED. We argue both the cases as follows.

Case 2.1: head $\left(\operatorname{fragment}\left(\widehat{\operatorname{node}}_{\pi}\right)\right)$. PRED $\in\{\& \operatorname{INCS}, \&$ ExIT $\}$.

By definition of $\mathcal{Q}, \pi \in \mathcal{Q}$. By Condition 19, there is an ordering $\pi_{1}, \pi_{2}, \ldots, \pi_{l}$ of the processes in $\mathcal{Q}$, and $\pi$ appears somewhere in that ordering. Assume for a contradiction that there is a run $R$ in which $\pi$ never completes the step at Line 25. Therefore, in $R$ there are some processes (including $\pi$ ) in $\mathcal{Q}$ that initiate the passage but never enter the CS. Since the processes never enter the $\mathrm{CS}$, after a certain configuration they are forever stuck at Line $\mathbf{2 5}$. Let $\pi_{j} \in \mathcal{Q}$ be the process in $R$ that forever loops at Line $\mathbf{2 5}$, such that it has the least index $j$ according to the ordering defined by Condition 19 . Let $C$ be the earliest configuration in $R$ such that all the processes appearing before $\pi_{j}$ in the ordering defined by Condition 19 have gone back to the Remainder section after completing the CS and $\pi_{j}$ is still stuck at Line 25. Since those processes are no more queued processes, $\pi_{j}$ appears first in the ordering, i.e., $\pi_{j}=\pi_{1}$. Since $P C_{\pi}=25$, by Condition 19c, $\widehat{\text { node }}_{\pi_{j}}$.PRED.CS_Signal $=1$ or $\left(\exists \pi^{\prime} \in \Pi, \pi_{j} \neq\right.$ $\pi^{\prime} \wedge \widehat{\operatorname{node}}_{\pi^{\prime}}=\widehat{\operatorname{node}}_{\pi_{j}}$.PRED $\wedge{\widehat{P C_{\pi^{\prime}}}}=\mathbf{2 8}$ ). If $\widehat{\text { node }}_{\pi_{j}}$.PRED.CS_Signal $=1$, then $\pi_{j}$ returns from the call to $\widehat{\text { node }}_{\pi_{j}}$.PRED.CS_SignAL.wait() at Line 25 completing the step. Otherwise, suppose $\widehat{\operatorname{node}}_{\pi_{j}}$.PRED.CS_SIgnal $\neq 1$ and $\left(\exists \pi^{\prime} \in\right.$ $\Pi, \pi_{j} \neq \pi^{\prime} \wedge \widehat{\operatorname{node}}_{\pi^{\prime}}=\widehat{\operatorname{node}}_{\pi_{j}}$.PRED $\wedge{\widehat{P C_{\pi^{\prime}}}}=$ 28). If $P C_{\pi^{\prime}}={\widehat{P C_{\pi^{\prime}}}}$, then $\pi^{\prime}$ eventually executes $\widehat{\text { node }}_{\pi^{\prime}}$.CS_SignAL.set () to completion at Line 28 and sets $\widehat{\operatorname{node}}_{\pi^{\prime}}$.CS_SIGNAL $=1$ in a wait-free manner. It follows that the call to $\widehat{\operatorname{node}}_{\pi_{j}}$.PRED.CS_SignaL.wait () at Line 25 returns subsequently in a wait-free manner. If $P C_{\pi^{\prime}} \neq \widehat{P C_{\pi^{\prime}}}$, then, by Condition 6, $P C_{\pi} \in\{\mathbf{1 0}\} \cup[\mathbf{1 6}$, 22. . By Condition 1. Node $\left[\right.$ port $\left._{\pi^{\prime}}\right] \neq$ NIL and $\widehat{\text { node }}_{\pi^{\prime}}$.PRED $=$ \&EXIT. Therefore, the if conditions at Lines $\mathbf{1 0}$ and $\mathbf{2 0}$ are not met, but the one at Line $\mathbf{2 1}$ is met and $\pi^{\prime}$ executes Line $\mathbf{2 8}$ as written in Line $\mathbf{2 2}$. Therefore, $\pi^{\prime}$ eventually executes $\widehat{\text { node }}_{\pi^{\prime}}$.CS_SignAL.set () to completion at Line 28 and sets $\widehat{\text { node }}_{\pi^{\prime}}$.CS_SigNAL $=1$ in a wait-free manner. It follows that the call to $\widehat{\text { node }}_{\pi_{j}}$.PRED.CS_SignAL.wait() at Line 25 returns subsequently in a wait-free manner. Thus $\pi_{j}$ eventually enters the CS by completing the remaining Try section at Line $\mathbf{2 6}$. This contradicts the assumption that $\pi_{j}$ is a process in $\mathcal{Q}$ with the least index $j$ defined by the order- 
ing by Condition 19. Therefore, we conclude that $\pi$ itself completes the step at Line 25 and eventually enters the CS.

Case 2.2: head $\left(\right.$ fragment $\left.\left(\widehat{\operatorname{rode}}_{\pi}\right)\right) . \mathrm{PRED} \in\{\mathrm{NIL}, \& \mathrm{CRASH}\}$.

Let $C$ be a configuration when $\widehat{P C_{\pi}}=\mathbf{2 5}$ and head $\left(\right.$ fragment $\left.\left(\widehat{\text { node }}_{\pi}\right)\right)$.PRED $\in$ $\{$ NIL, \&CRASH $\}$. By Condition 15, $\exists \pi_{i_{1}} \in \Pi, \pi_{i_{1}} \neq \pi \wedge{\widehat{P C_{\pi_{i_{1}}}}}=14 \wedge \widehat{\operatorname{node}}_{\pi_{i_{1}}}=$ head(fragment $\left.\left(\widehat{\operatorname{node}}_{\pi}\right)\right)$. Suppose $\widehat{\operatorname{node}}_{\pi_{i_{1}}}$. PRED $=$ NIL. By Condition 10, $P C_{\pi_{i_{1}}}=$ $\widehat{P C_{\pi_{i_{1}}}}$ (or $P C_{\pi_{i_{1}}} \in\{\mathbf{1 0}\} \cup[\mathbf{1 6}, \mathbf{1 8}$ ), we cover this case later). By Condition 13 . mypred $_{\pi_{i_{1}}} \in \mathcal{N}^{\prime}$. If $\pi_{i_{1}}$ takes normal steps at Line 14 , then it sets $\widehat{\operatorname{node}}_{\pi_{i_{1}}}$.PRED $=$ mypred $_{\pi_{i_{1}}}$ and sets $\widehat{P C_{\pi_{i_{1}}}}=\mathbf{1 5}$. We hold the argument for the current case when $\widehat{\operatorname{node}}_{\pi_{i_{1}}}$.PRED $=$ NIL briefly and argue the case when $\widehat{\text { node }}_{\pi_{i_{1}}}$. PRED $=\&$ CRASH as follows and then join the two arguments (i.e., $\widehat{\operatorname{node}}_{\pi_{i_{1}}}$.PRED $\in\{$ NIL, \&CRASH $\}$ ) later. So now assume that $\widehat{\operatorname{node}}_{\pi_{i_{1}}}$. PRED $=\&$ CRASH (this covers the case when $\widehat{\operatorname{node}}_{\pi_{i_{1}}}$.PRED $=$ NIL and $P C_{\pi_{i_{1}}} \in\left\{[\mathbf{1 0}\} \cup\left[\mathbf{1 6},[\mathbf{1 8})\right.\right.$, since $\widehat{\text { node }}_{\pi_{i_{1}}}$. PRED $=\&$ CRASH at Line 18 eventually). By Condition 10, $P C_{\pi_{i_{1}}} \in\{\mathbf{1 0}\} \cup[\mathbf{1 6}, \mathbf{2 1}, \cup[\mathbf{2 3}, \mathbf{2 4} \cup$ 30, 48. For every value of $P C_{\pi_{i_{1}}}$, it follows that $\pi_{i_{1}}$ eventually executes Line 49 (note, by Case 1 above, $\pi_{i_{1}}$ completes all steps at Line 35 ). Once $\pi_{i_{1}}$ executes Line 49 , it sets ${\widehat{P C_{\pi_{i_{1}}}}}_{25}$ 25. Hence, in both cases (i.e., $\widehat{\operatorname{node}}_{\pi_{i_{1}}}$.PRED $\in$ $\{\mathrm{NIL}, \& \mathrm{CRASH}\}) \widehat{P C_{\pi_{i_{1}}}}=\mathbf{2 5}$ eventually. Let $C^{\prime}$ be the earliest configuration after $C$ when $\widehat{P C_{\pi_{i_{1}}}}=2$ 25, by Condition 1 , $\widehat{\operatorname{node}}_{\pi_{i_{1}}} \cdot \operatorname{PRED} \in \mathcal{N}^{\prime}$ in $C^{\prime}$. If head $\left(\operatorname{fragment}\left(\widehat{\operatorname{node}}_{\pi}\right)\right)$.PRED $\{\& \mathrm{INCS}, \& \mathrm{EXIT}\}$ in $C^{\prime}$, then by the same argument as in Case 2.1 we are done. Otherwise, again by Condition $15, \exists \pi_{i_{2}} \in \Pi, \pi_{i_{2}} \neq \pi \wedge{\widehat{P C_{\pi_{i_{2}}}}}=14 \wedge \widehat{\operatorname{node}}_{\pi_{i_{2}}}=$ $\operatorname{head}\left(\operatorname{fragment}\left(\widehat{\operatorname{rode}}_{\pi}\right)\right)$.

We now show as follows that head(fragment $\left.\left(\widehat{\operatorname{node}}_{\pi}\right)\right)$.PRED $\in\{\& \operatorname{INCS}, \&$ ExIT $\}$ eventually. Assume to the contrary that head $\left(\operatorname{fragment}\left(\widehat{\operatorname{node}}_{\pi}\right)\right)$.PRED $\notin\{\& \mathrm{INCS}, \&$ ExIT $\}$ forever. We know there are $k$ active processes, and by Conditions 3, 4, and 7, there are a finite number of distinct fragments. Applying the above argument about $\pi$ and $\pi_{i_{1}}$ inductively on these fragments, the fragments increase in size monotonically and we get to a configuration such that each process satisfies one of three cases as follows: (i) the process has its node appear in fragment $\left(\widehat{\operatorname{node}}_{\pi}\right)$, (ii) there is a process $\pi_{i_{3}}$ such that head $\left(\right.$ fragment $\left.\left(\widehat{\operatorname{node}}_{\pi_{i_{3}}}\right)\right)$.PRED $\in\{\& \operatorname{INCS}, \&$ EXIT $\}$ and the process has its node appear in $\operatorname{fragment}\left(\widehat{\operatorname{node}}_{\pi_{i_{3}}}\right)$, or (iii) the process is in the Remainder section after completing the super-passage. Let $C^{\prime \prime}$ be the earliest such configuration. In $C^{\prime \prime}$ we have $\exists \pi_{i_{4}} \in \Pi, \pi_{i_{4}} \neq \pi \wedge \widehat{P C_{\pi_{i_{4}}}}=\left[14 \wedge \widehat{\operatorname{node}}_{\pi_{i_{4}}}=\right.$ head $\left(\right.$ fragment $\left.\left(\widehat{\operatorname{node}}_{\pi}\right)\right)$. We can now apply the above argument about $\pi$ and $\pi_{i_{1}}$ on $\pi$ and $\pi_{i_{4}}$. We continue to do so until we get to a configuration where each process satisfies one of the following two cases: (i) the process has its node appear in fragment $\left(\widehat{\operatorname{rode}}_{\pi}\right)$, (ii) the process forever remains in the Remainder section after completing the super-passage. Let $C^{\prime \prime \prime}$ be earliest such configuration where we have

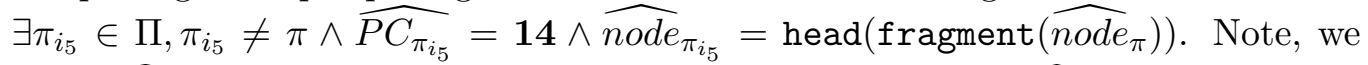
have $\widehat{P C_{\pi_{i_{5}}}}=14$ in $C^{\prime \prime \prime}$, and every other process $\pi^{\prime}$ has either $\widehat{P C_{\pi^{\prime}}}=\mathbf{1 1}$ (for being in the Remainder section) or $\widehat{P C_{\pi^{\prime}}} \in\{\underline{\mathbf{1 5}}, 25\}$ (for being in fragment $\left(\widehat{\operatorname{node}}_{\pi}\right)=$ 
fragment $\left.\left(\widehat{\operatorname{node}}_{\pi_{i_{5}}}\right)\right)$ for all configurations after $C^{\prime \prime \prime}$. We can apply the above argument about $\pi$ and $\pi_{i_{1}}$ on $\pi$ and $\pi_{i_{5}}$ so that $\exists \pi_{i_{6}} \in \Pi, \pi_{i_{6}} \neq \pi \wedge \widehat{P C_{\pi_{i_{6}}}}=$ 14 $\wedge \widehat{\text { node }}_{\pi_{i_{6}}}=\operatorname{head}\left(\right.$ fragment $\left.\left(\widehat{\text { node }}_{\pi}\right)\right)$. This contradicts the above conclusion that only $\widehat{P C_{\pi_{i_{5}}}}=14$ in all configurations after $C^{\prime \prime \prime}$. Therefore we conclude that our assumption that head $\left(\operatorname{fragment}\left(\widehat{\operatorname{node}}_{\pi}\right)\right)$.PRED $\notin\{\& \mathrm{INCS}, \&$ ExIT $\}$ forever is incorrect and head $\left(\operatorname{fragment}\left(\widehat{\operatorname{node}}_{\pi}\right)\right)$. PRED $\in\{\& \operatorname{INCS}, \&$ ExIT $\}$ eventually. Hence, by the same argument as in Case 2.1 we are done.

From the above it follows that $\pi$ completes the step at Line $\mathbf{2 5}$.

From the above it follows that $\pi$ completes the steps at Lines $\mathbf{2 5}$ and $\mathbf{3 5}$ whenever it encounters them during the passage. Therefore, the algorithm satisfies starvation freedom.

Lemma 6 (Wait-free Exit). There is a bound b such that, if a process $\pi$ is in the Exit section, and executes steps without crashing, $\pi$ completes the Exit section in at most $b$ of its steps.

Proof. An inspection of the algorithm reveals that Lines $27, \mathbf{2 9}$ do not involve repeated execution of any steps. The implementation of the Signal object from Figure 2 shows that the code for $\mathcal{X}$.set () does not involve a loop, Hence, the call to mynode ${ }_{\pi}$.CS_Signal.set () at Line 28 terminates. Hence the claim.

Lemma 7 (Wait-Free CSR). There is a bound b such that, if a process crashes while in the CS, it reenters the CS before completing $b$ consecutive steps without crashing.

Proof. Suppose $\pi$ crashes while in the CS, i.e., when $\widehat{P C_{\pi}}=$ 27, $\pi$ crashes. By Condition 1 . NoDE $\left[\right.$ port $\left._{\pi}\right] \neq \mathrm{NIL}$ and $\widehat{\operatorname{node}}_{\pi}$.PRED $=\&$ INCS. Therefore, when $\pi$ restarts from the crash and starts executing at Line $\mathbf{1 0}$, it finds that the if conditions at Lines $\mathbf{1 0}$ and $\mathbf{1 8}$ are not met. It therefore reaches Line 20 with mypred $_{\pi}=\widehat{\text { node }}_{\pi}$. PRED (by Condition 2 ) by executing Lines $\mathbf{1 0}$. 16 (none of which are repeatedly executed). The if condition at Line 20 is met and $\pi$ is put into the CS in a wait-free manner. Hence the claim.

Lemma 8 (Critical Section Reentry). If a process $\pi$ crashes inside the CS, then no other process enters the CS before $\pi$ reenters the CS.

Proof. This is immediate from Lemma 4 and Lemma 7 as observed in $[9]$.

\section{Proof of correctness of Signal object}

Proof of Theorem 1. Let $\alpha$ be the earliest event where some process performed Line $\mathbf{1}$ and $\beta$ be the earliest event where some process $\pi^{\prime}$ performed Line 8 and does not subsequently fail.

Case 1: $\alpha$ occurs before $\beta$.

In this case we linearize the execution as follows:

- every execution of $\mathcal{X}$.set () is linearized to its Line $\mathbf{1}$.

- every execution of $\mathcal{X}$.wait () is linearized to its Line 8 , where $\mathcal{X}$.STATE is 1 (since $\alpha$ precedes $\beta)$. 
Since $\alpha$ occurs before $\beta, \pi^{\prime}$ notices that BIT $=1$ at Line 8 and hence returns from the call to wait () .

Case 2: $\beta$ occurs before $\alpha$.

Consider the execution of $\mathcal{X}$.set () that is the first to complete. Let $\pi$ be the process that performs this execution of $\mathcal{X}$.set () . At Line $2 \pi$ reads $g o_{\pi^{\prime}}$ from GoADDR into $a d d r_{\pi}$. Since $\beta$ occurs before $\alpha, a d d r_{\pi} \neq$ NIL, therefore, at Line $4 \pi$ sets $* g o_{\pi^{\prime}}$ to true. This releases $\pi^{\prime}$ from its busy-wait at Line 9 . We linearize the call to $\mathcal{X}$.set () by $\pi$ to its Line 4 , and every other complete execution of $\mathcal{X}$.set () in the run to its point of completion. Note, $\beta$ is the earliest event where some process $\pi^{\prime}$ performed Line 8 and does not subsequently fail and we assume that no two executions of $\mathcal{X}$.wait () are concurrent. Therefore, every other execution of $\mathcal{X}$.wait () , happens after the call considered in $\alpha$ sets BIT to 1 . This implies that such a call would complete because the calling process would read BiT = 1 at Line 8 and return.

RMR Complexity: It is easy to see that the RMR Complexity of $\mathcal{X}$.set () is $O(1)$ since there are a constant steps in any execution of $\mathcal{X} \cdot \operatorname{set}()$. For any execution of $\mathcal{X}$.wait () by a process $\pi$, $\pi$ creates a new boolean at Line 5 that resides in $\pi$ 's memory partition. Therefore, the busy-wait by $\pi$ at Line 9 incurs a $O(1)$ RMR and the rest of the lines in $\mathcal{X}$.wait() (Lines 5 , 8) incur a $O(1)$ RMR.

\section{E Proof of invariant}

In this section we prove that our algorithm from Figures 34 satisfies the invariant described in Figures 8 11. In order to prove that we need support from a few extra conditions that we present in Figures 12 14. Therefore, we prove that our algorithm satisfies all the conditions described in Figures 8.14.

Lemma 9. The algorithm in Figures 3 4 satisfies the invariant (i.e., the conjunction of the 39 conditions) stated in Figures 8 14, i.e., the invariant holds in every configuration of every run of the algorithm.

Proof. We prove the lemma by induction. Specifically, we show (i) base case: the invariant holds in the initial configuration, and (ii) induction step: if the invariant holds in a configuration $C$ and a step of a process takes the configuration $C$ to $C^{\prime}$, then the invariant holds in $C^{\prime}$.

In the initial configuration, we have TAIL $=\&$ SPECIALNode, $\forall \pi \in \Pi, P C_{\pi}=\mathbf{1 0}, \widehat{P C_{\pi}}=\mathbf{1 1}$. and $\operatorname{Node}\left[\widehat{\text { port }_{\pi}}\right]=$ NIL. Note, $|\mathcal{Q}|=0$ by definition of $\mathcal{Q}$. Since all processes are in the Remainder section, Condition 1 holds because of the value of the NoDE array as noted above. Since $\widehat{\text { node }}_{\pi}=$ NIL by definition, Condition 4, 7 holds. Since TAIL $=\&$ SpecialNode, Condition 16 holds. Since $|\mathcal{Q}|=0$, TAIL $=\&$ SpecialNode as noted above, hence, Condition 18 holds. All of the remaining conditions of the invariant hold vacuously in the initial configuration. Hence, we have the base case.

To verify the induction step, Let $C$ be an arbitrary configuration in which the invariant holds, $\pi$ be an arbitrary process, and $C^{\prime}$ be the configuration that results when $\pi$ takes a step from $C$. In the following, we enumerate each possible step of $\pi$ and argue that the invariant continues to hold in $C^{\prime}$, even though the step changes the values of some variables. Since our invariant involves universal quantifiers for all the conditions, we have only argued it thoroughly as it is applicable to $\pi$ and wherever necessary for another process for the sake of brevity. We also skip arguing about conditions that hold vacuously, are easy to verify, are argued before in a similar way, or need not be argued if the step does not affect the condition. Induction step due to a crash step of $\pi$ is argued 
Definitions (Continued from Figure 9):

- owner(qnode) denotes the process that created the qnode at Line $\mathbf{1 1}$

Conditions (Continued from Figure 11):

20. $\forall \pi \in \Pi,\left(P C_{\pi}=31 \wedge \operatorname{head}(\right.$ fragment $(\mathrm{TAIL}))$. PRED $\left.\in\{\& \mathrm{INCS}, \& \mathrm{ExIT}\}\right) \Rightarrow$ fragment $\left(\widehat{\operatorname{rode}}_{\pi}\right) \neq$ fragment $($ TAIL $)$

21. $\forall \pi \in \Pi,\left(P C_{\pi} \in \mathbf{3 2} 41 \Rightarrow\right.$ tailpath $\left._{\pi}=\mathrm{NIL}\right) \wedge\left(P C_{\pi} \in \mathbf{3 2} \mathbf{4 1} \Rightarrow\right.$ headpath $\left._{\pi}=\mathrm{NIL}\right)$

$\wedge\left(P C_{\pi}=\mathbf{3 2} \Rightarrow i_{\pi} \in[0, k]\right) \wedge\left(P C_{\pi} \in \mathbf{3 3}, \mathbf{3 8} \Rightarrow i_{\pi} \in[0, k-1]\right)$

$\wedge\left(P C_{\pi} \in \mathbf{3 9} 49 \Rightarrow i_{\pi}=k\right) \wedge\left(P C_{\pi} \in \mathbf{3 2} 4 \mathbf{4 9} \Rightarrow \operatorname{tail}_{\pi} \in \mathcal{N}^{\prime}\right)$

22. $\forall \pi \in \Pi, P C_{\pi} \in \mathbf{3 2} \mathbf{3 9} \Rightarrow\left(\right.$ tail $_{\pi} \in V_{\pi} \vee\left(\exists i \in\left[i_{\pi}, k-1\right]\right.$, tail $\left._{\pi}=\operatorname{NoDE}[i]\right) \vee\left(\right.$ tail $\left.\left._{\pi} . \operatorname{PrED}=\& \mathrm{ExIT}\right)\right)$

23. $\forall \pi \in \Pi$, if $P C_{\pi} \in \mathbf{3 2}$ 49, then:

(a) $\left(V_{\pi}, E_{\pi}\right)$ is a directed acyclic graph,

(b) Maximal paths in $\left(V_{\pi}, E_{\pi}\right)$ are disjoint.

24. $\forall \pi \in \Pi$, if $P C_{\pi} \in \mathbf{3 2}, 49$, then one of the following holds (i.e., (a) $\vee(\mathrm{b}) \vee(\mathrm{c}) \vee(\mathrm{d})$ ):

(a) head(fragment $\left(\right.$ tail $\left.\left._{\pi}\right)\right) . P R E D \in\{\& I N C S, \& E x I T\}$

(b) there is a unique maximal path $\sigma$ in the graph $\left(V_{\pi}, E_{\pi}\right)$, such that, end $(\sigma)$.PRED $\in\{\& \operatorname{InCS}, \& \operatorname{ExIT}\}$ and $\operatorname{start}(\sigma)$. PRED $\neq \&$ ExIT

(c) $i_{\pi}<k$ and $\exists i^{\prime} \in\left[i_{\pi}, k-1\right]$, Node $\left[i^{\prime}\right]$.Pred.Pred $\in\{\& \operatorname{InCS}, \&$ Exit $\}$ $\wedge$ tail (fragment $\left.\left(\operatorname{NoDE}\left[i^{\prime}\right]\right)\right) . P R E D \neq \&$ EXIT

(d) $|\mathcal{Q}|=0$

25. $\forall \pi \in \Pi,\left(P C_{\pi} \in \mathbf{3 2} 39 \wedge \operatorname{head}\left(\right.\right.$ fragment $\left(\right.$ tail $\left.\left._{\pi}\right)\right)$. PRED $\in\{\& \operatorname{INCS}, \&$ EXIT $\left.\}\right) \Rightarrow$

$\forall \widehat{\operatorname{node}}_{\pi^{\prime}} \in \operatorname{fragment}\left(\widehat{\operatorname{node}}_{\pi}\right),\left(\left(\exists i_{\pi}<i^{\prime}<k, \operatorname{NoDE}\left[i^{\prime}\right]=\widehat{\operatorname{node}}_{\pi^{\prime}}\right) \vee\right.$

$\left(\widehat{\operatorname{node}}_{\pi^{\prime}} \in V_{\pi} \wedge\left(\widehat{\operatorname{node}}_{\pi} \neq \widehat{\operatorname{node}}_{\pi^{\prime}} \Rightarrow\left(\widehat{\operatorname{node}}_{\pi^{\prime}}, \widehat{\operatorname{node}}_{\pi^{\prime}}\right.\right.\right.$.PRED $\left.\left.) \in E_{\pi}\right)\right)$

26. $\forall \pi \in \Pi$, If $P C_{\pi} \in \mathbf{3 2}$ 41 and there is a maximal path $\sigma$ in $\left(V_{\pi}, E_{\pi}\right)$ such that end $(\sigma)$.PRED $\in$ $\{\& \mathrm{INCS}, \& \mathrm{ExIT}\}$, then, for an arbitrary vertices $v$ and $v^{\prime}$ on the path $\sigma$,

$\forall \widehat{\text { node }} \in \operatorname{fragment}(v),\left(\left(\exists i_{\pi}<i^{\prime}<k, \operatorname{NoDE}\left[i^{\prime}\right]=\widehat{\text { node }}\right) \vee\left(\widehat{\text { node }} \in V_{\pi}\right.\right.$

$\wedge(\widehat{\text { node }}$. PrED $\left.\left.\left.\notin\{\& \operatorname{InCS}, \& \mathrm{ExIT}\} \Rightarrow \widehat{(\text { node }}, \widehat{\text { node }} . \mathrm{PRED}) \in E_{\pi}\right)\right)\right)$,

and $\left(\operatorname{fragment}(v) \neq \operatorname{fragment}\left(v^{\prime}\right) \Rightarrow\right.$

$\left.\left(v . \operatorname{Pred} \in\{\& \operatorname{InCS}, \& \operatorname{ExIT}\} \vee v^{\prime} . \operatorname{PrED} \in\{\& \operatorname{InCS}, \& \operatorname{ExIT}\}\right)\right)$

Figure 12: (Continued from Figure 11.) Invariant for the $k$-ported recoverable mutual exclusion algorithm from Figures 3n4. (Continued in Figure 13.)

in the end. To aid in reading we have numbered each step according to the value of the program counter wherever possible. For the purpose of this proof we assume that $\pi$ executes the algorithm using the port $p$, hence $\widehat{\text { port }_{\pi}}=p$.

10 (a). $\pi$ executes Line 10 when $\widehat{P C_{\pi}} \in\{\mathbf{1 1}, 12\}$.

In $C, P C_{\pi}=10$ and $\widehat{P C_{\pi}} \in\{1 \mathbf{1 1}, 12\}$. By Condition 1, NoDE $\widehat{\text { port }_{\pi}}=$ NIL.

The if condition at Line $\mathbf{1 0}$ evaluates to true. Therefore, this step changes $P C_{\pi}$ and $\widehat{P C_{\pi}}$ to 11.

Condition 1. As argued above, $\widehat{P C_{\pi}}=\left[11\right.$ and NoDE $[\widehat{p o r t}]=$ NIL in $C^{\prime}$. Therefore the condition holds in $C^{\prime}$.

10 (b). $\pi$ executes Line 10 when $\widehat{P C_{\pi}} \notin\{\mathbf{1 1}, \mathbf{1 2}\}$.

In $C, P C_{\pi}=10$ and $\widehat{P C_{\pi}} \notin\{1 \mathbf{1 1}, 12]$. By Condition 1 , NoDE $\left.\widehat{\text { port }_{\pi}}\right] \neq$ NIL.

The if condition at Line $\mathbf{1 0}$ evaluates to false. Therefore, this step changes $P C_{\pi}$ to $\mathbf{1 6}$.

The step does not affect any condition, so the invariant continues to hold in $C^{\prime}$.

11. $\pi$ executes Line 11 


\section{Conditions (Continued from Figure 11):}

27. $\forall \pi \in \Pi$, if $P C_{\pi} \in \mathbf{3 2} 4 \mathbf{4 9}$, then:

(a) $\forall v \in V_{\pi}, v \in \mathcal{N}^{\prime} \wedge\left(i_{\pi}>\widehat{\text { port }_{\pi}} \Rightarrow\right.$ mynode $\left._{\pi} \in V_{\pi}\right)$

(b) $\forall 0 \leq i^{\prime}<i_{\pi},\left(\operatorname{NodE}\left[i^{\prime}\right] \in \operatorname{fragment}\left(\widehat{\operatorname{node}}_{\pi}\right) \wedge \operatorname{fragment}\left(\right.\right.$ tail $\left.\left._{\pi}\right) \neq \operatorname{fragment}\left(\widehat{\operatorname{node}}_{\pi}\right)\right) \Rightarrow \operatorname{NoDE}\left[i^{\prime}\right] \in V_{\pi}$

(c) $\forall 0 \leq i^{\prime}<i_{\pi}, \forall v \in V_{\pi},\left(\underset{p o r t t_{\text {ouner }}(v)}{v}=i^{\prime} \wedge v \neq \operatorname{NodE}\left[i^{\prime}\right]\right) \Rightarrow\left(v \cdot \operatorname{PreD}=\& \operatorname{ExiT} \wedge \forall p^{\prime} \in \mathcal{P}, \operatorname{Node}\left[p^{\prime}\right] \neq v\right)$

(d) $\forall 0 \leq i^{\prime}<i_{\pi},\left(\operatorname{Node}\left[i^{\prime}\right]\right.$ Pred $\left.\notin\{\& \mathrm{Crash}, \& \operatorname{InCS}, \& \operatorname{ExiT}\} \wedge \operatorname{Node}\left[i^{\prime}\right] \in V_{\pi}\right) \Rightarrow$

$\left(\operatorname{Node}\left[i^{\prime}\right], \operatorname{Node}\left[i^{\prime}\right] . \operatorname{Pred}\right) \in E_{\pi}$

(e) $\forall 0 \leq i^{\prime}<i_{\pi},\left(\left(\forall v \in V_{\pi}, \operatorname{Node}\left[i^{\prime}\right] \neq v\right) \Rightarrow\right.$

$\left(\left(\right.\right.$ head $\left(\right.$ fragment $\left(\right.$ tail $\left.\left._{\pi}\right)\right) . \operatorname{PrED} \in\{\& \operatorname{INCS}, \& \operatorname{ExIT}\} \wedge \operatorname{NODE}\left[i^{\prime}\right] \in$ fragment $\left(\right.$ tail $\left.\left._{\pi}\right)\right)$ $\vee$ head $\left(\right.$ fragment $\left.\left(\operatorname{NODE}\left[i^{\prime}\right]\right)\right)$. PrED $\in\{$ NIL,$\&$ CrASH $\left.\left.\}\right)\right)$

(f) $\forall v \in V_{\pi}$, end $(v) \cdot \operatorname{PrED}=\&$ Crash $\Rightarrow$ head $($ fragment $(v))$.PrED $=\&$ Crash

(g) $\forall v \in V_{\pi}$, If there is a pair $(v, u) \in E_{\pi}$, then $u \in V_{\pi}$ and $(v$.PRED $=u \vee v \cdot \operatorname{PrED} \in\{\& \operatorname{INCS}, \& \operatorname{ExIT}\})$

(h) $\forall(u, v) \in E_{\pi},(\exists i \in[0, k-1], \operatorname{Node}[i]=u) \vee\left(\forall i^{\prime} \in[0, k-1], \operatorname{Node}\left[i^{\prime}\right] \cdot \operatorname{Pred} \neq v\right)$

(i) $\forall(v, w) \in E_{\pi},(v \cdot \operatorname{PrED} \in\{w, \& \operatorname{InCS}, \& \operatorname{ExIT}\}) \wedge(v \cdot \operatorname{Pred} \in\{\& \operatorname{InCS}, \& \operatorname{EXIT}\} \Rightarrow w \cdot \operatorname{PrED}=\& \operatorname{ExIT})$

(j) $\forall(u, v) \in E_{\pi}, u \neq$ mynode $_{\pi}$

(k) $i_{\pi}>\widehat{\text { port }_{\pi}} \Rightarrow$ there is a path $\sigma$ in the graph $\left(V_{\pi}, E_{\pi}\right)$, such that, end $(\sigma)=$ mynode $_{\pi}$.

28. $\forall \pi \in \Pi,\left(P C_{\pi} \in \mathbf{3 2} 4 \mathbf{4 7} \wedge\right.$ head $\left(\right.$ fragment $\left(\right.$ tail $\left.\left._{\pi}\right)\right)$.PrED $\left.\in\left\{\& \mathrm{INCS}_{\mathrm{N}} \& \mathrm{ExIT}\right\}\right) \Rightarrow$ fragment $\left(\widehat{\text { node }}_{\pi}\right) \neq$ fragment (TAIL)

$\wedge\left(\left(P C_{\pi} \in \mathbf{3 9} 4 \mathbf{4 7}\right) \wedge\right.$ tail $\left._{\pi} \notin V_{\pi}\right) \Rightarrow$ head $\left(\operatorname{fragment}\left(\right.\right.$ tail $\left.\left._{\pi}\right)\right)$. PRED $\left.\in\{\& \operatorname{INCS}, \& \operatorname{ExIT}\}\right)$

$\wedge\left(\left(P C_{\pi} \in \mathbf{4 2}\right.\right.$ 47 $) \wedge$ tailpath $\left._{\pi} \neq \mathrm{NIL}\right) \Leftrightarrow$ tail $\left._{\pi} \in V_{\pi}\right)$

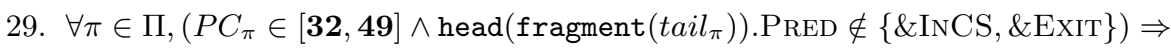
head(fragment(TAIL)).PRED $\notin\{\&$ INCS, \&EXIT $\}$

30. $\forall \pi \in \Pi,\left(P C_{\pi}=34 \Rightarrow\left(\right.\right.$ cur $_{\pi}=\operatorname{NIL} \vee\left(\right.$ cur $_{\pi} \in \mathcal{N}^{\prime} \wedge\left(\right.$ cur $_{\pi}=\operatorname{Node}\left[i_{\pi}\right] \vee c u r_{\pi}$. PrED $=\&$ Exit $\left.\left.\left.)\right)\right)\right)$

$\wedge\left(P C_{\pi}=\overline{35} \Rightarrow\left(\right.\right.$ cur $_{\pi} \cdot$ NONNIL_SignAL $=1 \vee\left(\exists \pi^{\prime} \in \Pi, \pi \neq \pi^{\prime} \wedge\right.$ cur $_{\pi}=\widehat{\text { node }_{\pi^{\prime}}} \wedge \widehat{P C_{\pi^{\prime}}} \in[13$ 15] $\left.)\right)$

$\wedge\left(P C_{\pi} \in \mathbf{3 5}\left[\mathbf{3 8} \Rightarrow\left(\right.\right.\right.$ cur $_{\pi} \in \mathcal{N}^{\prime} \wedge\left(\right.$ cur $_{\pi}=\operatorname{NODE}\left[i_{\pi}\right] \vee$ cur $\left.\left.\left._{\pi} \cdot \mathrm{PRED}=\& \mathrm{EXIT}\right)\right)\right)$

$\wedge\left(P C_{\pi} \in \mathbf{3 6}\right] \mathbf{3 7} \Rightarrow$ cur $_{\pi}$. Pred $\in\{\&$ Crash, $\&$ InCS, \&EXIT $\left.\} \cup \mathcal{N}^{\prime}\right) \wedge\left(P C_{\pi}=\overline{\mathbf{3 8}} \Rightarrow\right.$ curpred $\left._{\pi} \in \mathcal{N}^{\prime}\right)$

31. $\forall \pi \in \Pi,\left(\left(P C_{\pi} \in 42,47 \wedge t_{\text {ailpath }} \neq\right.\right.$ NIL $\wedge$ end $\left(\right.$ tailpath $\left._{\pi}\right)$.PRED $\notin\{\&$ INCS, \&EXIT $\left.\left.\}\right) \vee P C_{\pi}=48\right) \Rightarrow$ head $\left(\operatorname{fragment}\left(\operatorname{tail}_{\pi}\right)\right)$.PRED $\notin\{\&$ INCS, \&ExIT $\}$

32. $\forall \pi \in \Pi$, if $P C_{\pi} \in 3941$ and head $\left(\operatorname{fragment}\left(\right.\right.$ tail $\left.\left._{\pi}\right)\right)$.PRED $\in\left\{\& \mathrm{INCS}_{\mathrm{N}} \& \mathrm{ExIT}\right\}$, then there is a maximal path $\sigma$ in the graph $\left(V_{\pi}, E_{\pi}\right)$ such that:

(a) $\operatorname{end}(\sigma)=$ mynode $_{\pi}$

(b) $\operatorname{start}(\sigma)=\operatorname{tail}\left(\right.$ fragment $\left(\right.$ mynode $\left.\left._{\pi}\right)\right)$

33. $\forall \pi \in \Pi$, if $P C_{\pi} \in$ 41 49, then mypath $_{\pi}$ is the unique path in Paths $s_{\pi}$ such that mynode appears in mypath $_{\pi}$

34. $\forall \pi \in \Pi$, if $P C_{\pi}=39$ and for every maximal path $\sigma$ in $\left(V_{\pi}, E_{\pi}\right), \neg(\operatorname{end}(\sigma)$.PrED $\in\{\& \operatorname{INCS}, \&$ ExIT $\}$

$\wedge \operatorname{start}(\sigma) . \operatorname{PreD} \neq \&$ ExiT $)$, then $\left(\right.$ head $\left.\left(\operatorname{fragment}\left(\operatorname{tail}_{\pi}\right)\right) . \operatorname{Pred} \in\{\& \operatorname{InCS}, \& \operatorname{ExIT}\} \vee|\mathcal{Q}|=0\right)$

Figure 13: (Continued from Figure 12,) Invariant for the $k$-ported recoverable mutual exclusion algorithm from Figures 3.4. (Continued in Figure 14.)

In $C, P C_{\pi}=$ 11. By Condition 6, $\widehat{P C_{\pi}} \in$ 11, 12 in $C$. By definition, $\widehat{\operatorname{node}}_{\pi}=$ NIL in $C$.

This step creates a new QNode in the shared memory which gets included in the set $\mathcal{N}$. The node gets a unique address and the variable mynode $_{\pi}$ holds the address of this new node. This step also initializes this new node so that mynode $_{\pi}$.PRED $=$ NIL, mynode ${ }_{\pi}$.NONNIL_SigNAL $=$ 0 , and mynode ${ }_{\pi}$ CS_Signal $=0$. The step then changes $P C_{\pi}$ and $\widehat{P C_{\pi}}$ to $\mathbf{1 2}$.

Condition 4. As argued above, the step creates a new QNode in shared memory that $\widehat{n o d e}_{\pi}$ is pointing to in $C^{\prime}$. The step also initializes $\widehat{\operatorname{node}}_{\pi}$. PRED to NIL. Therefore, the condition holds in $C^{\prime}$. 


\section{Conditions (Continued from Figure 11):}

35. $\forall \pi \in \Pi$, if $P C_{\pi}=39$ and there is a maximal path $\sigma$ in $\left(V_{\pi}, E_{\pi}\right)$, such that, end $(\sigma) . \operatorname{PRED} \in\{\& \operatorname{INCS}, \& \operatorname{ExIT}\} \wedge \operatorname{start}(\sigma) \cdot \operatorname{PRED} \neq \&$ EXIT, then

$$
\begin{aligned}
& \vee \\
& \vee \exists \text { node } \in \mathcal{N},(\widehat{\text { node }}=\operatorname{start}(\sigma) \wedge \widehat{\text { node }}=\operatorname{tail}(\text { fragment }(\widehat{\text { node }})) \\
& \wedge \text { head }(\text { fragment }(\widehat{\text { node }})) . \text { PRED } \in\{\& \operatorname{INCS}, \& \operatorname{ExIT}\} \\
& \left.\left.\wedge \operatorname{fragment}\left(\widehat{\text { node }_{\pi}}\right) \neq \operatorname{fragment}(\widehat{\text { node }})\right)\right)
\end{aligned}
$$

36. $\forall \pi \in \Pi,\left(P C_{\pi} \in\{44,45\} \Rightarrow \operatorname{end}\left(\sigma_{\pi}\right)\right.$. PRED $\left.\in\{\& \operatorname{INCS}, \& \operatorname{ExIT}\}\right)$

$$
\wedge\left(P C_{\pi}=45 \Rightarrow\left(\left|\sigma_{\pi}\right|>1 \wedge \operatorname{start}\left(\sigma_{\pi}\right)=\operatorname{tail}\left(\operatorname{fragment}\left(\operatorname{start}\left(\sigma_{\pi}\right)\right)\right)\right)\right.
$$

37. $\forall \pi \in \Pi,\left(P C_{\pi} \in 40,48 \wedge\right.$ headpath $\left.h_{\pi}=\mathrm{NIL}\right) \Rightarrow$

$\left(\right.$ head $\left(\right.$ fragment $\left(\right.$ tail $\left.\left._{\pi}\right)\right)$. PrED $\in\{\& \operatorname{InCS}, \&$ ExIT $\} \vee|\mathcal{Q}|=0$

$\vee$ (There is a maximal path $\sigma$ in $\left(V_{\pi}, E_{\pi}\right)$, such that, $\operatorname{end}(\sigma)$. Pred $\in\{\& \operatorname{InCS}, \&$ ExIT $\} \wedge \operatorname{start}(\sigma)$. Pred $\neq \&$ ExIT, and $\exists \widehat{\text { node }} \in \mathcal{N}, \widehat{(\text { node }}=\operatorname{start}(\sigma) \wedge \widehat{\text { node }}=\operatorname{tail}($ fragment $(\widehat{\text { node }}))$

$$
\begin{aligned}
& \wedge \operatorname{head}(\operatorname{fragment}(\widehat{\text { node }})) . \text { PRED } \in\{\& \text { INCS }, \& \text { EXIT }\} \\
& \left.\left.\left.\wedge \operatorname{fragment}\left(\widehat{\text { node }_{\pi}}\right) \neq \operatorname{fragment}(\widehat{\text { node }})\right)\right)\right)
\end{aligned}
$$

38. $\forall \pi \in \Pi,\left(P C_{\pi} \in 40,48 \wedge\right.$ headpath $\left.h_{\pi} \neq \mathrm{NIL}\right) \Rightarrow$

$$
\begin{aligned}
& \left(\left(\exists \sigma \in \text { Path }_{\pi}, \text { headpath }_{\pi}=\sigma\right) \wedge \text { head }\left(\text { fragment }\left(\text { tail }_{\pi}\right)\right) . \operatorname{PrED} \in\{\& \operatorname{INCS}, \& \operatorname{ExIT}\}\right. \\
& \vee \exists \widehat{\text { node }} \in \mathcal{N},\left(\widehat{\text { node }}=\operatorname{start}\left(\text { headpath }_{\pi}\right) \wedge \widehat{\text { node }}=\operatorname{tail}(\text { fragment }(\widehat{\text { node }}))\right. \\
& \wedge\left(\widehat{\text { node }} . \text { Pred }=\& \operatorname{INCS} \Rightarrow\left(\exists \pi^{\prime} \in \Pi, \pi \neq \pi^{\prime} \wedge \widehat{P_{\pi^{\prime}}}=\underline{\mathbf{2 7}} \wedge \widehat{\text { node }}=\widehat{\text { node }_{\pi^{\prime}}}\right)\right) \\
& \wedge(\widehat{\text { node }} . \text { PRED }=\& \text { EXIT } \Rightarrow|\mathcal{Q}|=0) \\
& \wedge(\widehat{\text { node }} \text {.Pred } \notin\{\& \text { InCS }, \& \text { EXIT }\} \Rightarrow \\
& \left(\exists \pi^{\prime} \in \Pi, \pi \neq \pi^{\prime} \wedge \widehat{P C_{\pi^{\prime}}} \in\left\{[\mathbf{1 5}\} \cup[\mathbf{2 5}, \mathbf{2 6}] \wedge \widehat{\text { node }}=\widehat{\text { node }}_{\pi^{\prime}}\right)\right) \\
& \wedge \text { head }(\text { fragment }(\widehat{\text { node }})) . \text { PRED } \in\{\& \text { INCS, \&EXIT }\} \\
& \left.\left.\wedge \text { fragment }\left(\widehat{\text { node }}_{\pi}\right) \neq \text { fragment }(\widehat{\text { node }})\right)\right)
\end{aligned}
$$

39. $\forall \pi \in \Pi, P C_{\pi}=49 \Rightarrow\left(\widehat{P C_{\pi}}=14 \wedge\right.$ mypred $_{\pi} \in \mathcal{N}^{\prime} \wedge$ mypred $_{\pi}=\operatorname{tail}\left(\right.$ fragment $\left(\right.$ mypred $\left.\left._{\pi}\right)\right)$

$$
\begin{aligned}
& \wedge\left(\text { mypred }_{\pi} \cdot \text { PRED }=\& \text { INCS } \Rightarrow\left(\exists \pi^{\prime} \in \Pi, \pi \neq \pi^{\prime} \wedge \widehat{P C_{\pi^{\prime}}}=\left[\mathbf{2 7} \wedge \text { mypred }_{\pi}=\widehat{\text { node }}_{\pi^{\prime}}\right)\right)\right. \\
& \wedge\left(\text { mypred } _ { \pi } \cdot \text { PRED } = \& \text { EXIT } \Rightarrow \left(\left(\exists \pi^{\prime} \in \Pi, \pi \neq \pi^{\prime} \wedge \widehat{P C_{\pi^{\prime}} \in[\mathbf{2 8}] \mathbf{2 9}}\right.\right.\right.
\end{aligned}
$$$$
\left.\left.\left.\left.\wedge \text { mypred }_{\pi}=\widehat{\text { node }}_{\pi^{\prime}}\right) \vee\left(\forall p^{\prime} \in \mathcal{P}, \operatorname{NoDE}\left[p^{\prime}\right] \neq \text { mypred }_{\pi}\right)\right) \wedge|\mathcal{Q}|=0\right)\right)
$$

$\wedge$ mypred $_{\pi} \cdot$ Pred $\notin\{\&$ InCS, \&EXIT $\} \Rightarrow$

$$
\left(\exists \pi^{\prime} \in \Pi, \pi \neq \pi^{\prime} \wedge \widehat{P C_{\pi^{\prime}}} \in\left[\mathbf{1 4}[\mathbf{1 5}] \cup\left[\mathbf{2 5}\left[\mathbf{2 6} \wedge \wedge \text { mypred }_{\pi}=\widehat{\text { node }}_{\pi^{\prime}}\right)\right)\right.\right.
$$

$\wedge\left(\right.$ head $\left(\right.$ fragment $\left(\right.$ mypred $\left.\left._{\pi}\right)\right)$. PRED $\in\{$ NIL,$\&$ CRASH $\} \Rightarrow\left(\exists \pi^{\prime} \in \Pi, \pi \neq \pi^{\prime} \wedge \widehat{P C_{\pi^{\prime}}}=14\right.$ $\wedge$ mypred $\left.\left._{\pi}=\operatorname{tail}\left(\operatorname{fragment}\left(\widehat{\text { node }}_{\pi^{\prime}}\right)\right) \wedge \widehat{\text { node }}_{\pi^{\prime}}=\operatorname{head}\left(\operatorname{fragment}\left(\widehat{\text { node }}_{\pi^{\prime}}\right)\right)\right)\right)$

$\wedge$ fragment $\left(\widehat{\text { node }}_{\pi}\right) \neq$ fragment $\left(\right.$ mypred $\left.\left._{\pi}\right)\right)$

Figure 14: (Continued from Figure 13.) Invariant for the $k$-ported recoverable mutual exclusion algorithm from Figures 3 . 4.

Condition 8. Since the step creates a new node in shared memory, no process has a reference to the node except for $\pi$ in $C^{\prime}$. Therefore, $\forall q \in \mathcal{P}, \operatorname{NodE}[q] \neq$ mynode $_{\pi} \wedge$ Node $[q]$.PRED $\neq$ mynode $_{\pi}$. Since the nothing except mynode $_{\pi}$ has a reference to the new node, mynode $_{\pi}=$

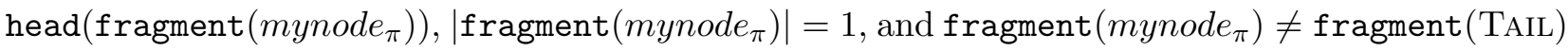
holds. Also, as observed above, mynode $_{\pi}$.NonNIL_SignAL $=0$, and mynode $e_{\pi}$ CS_SignaL $=0$ in $C^{\prime}$. Therefore, the condition holds in $C^{\prime}$.

12. $\pi$ executes Line 12 .

In $C, P C_{\pi}=\mathbf{1 2}$ and $\widehat{P C_{\pi}}=\mathbf{1 2}$, By Condition 8, mynode $e_{\pi} \in \mathcal{N}^{\prime}, \forall q \in \mathcal{P}, \operatorname{NoDE}[q] \neq$ mynode $_{\pi} \wedge \operatorname{NODE}[q]$. PRED $\neq$ mynode $_{\pi}$, mynode $_{\pi}$. PRED $=$ NIL, fragment $\left(\right.$ mynode $\left._{\pi}\right) \neq$

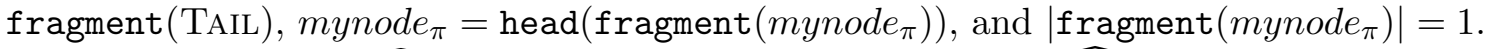

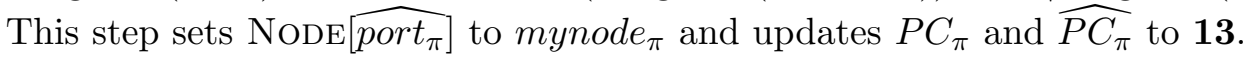


Condition 3. As argued above, mynode $_{\pi} \in \mathcal{N}^{\prime}$, mynode $_{\pi} \cdot$ PRED $=$ NIL, and $\mid$ fragment $\left(\right.$ mynode $\left._{\pi}\right) \mid=$

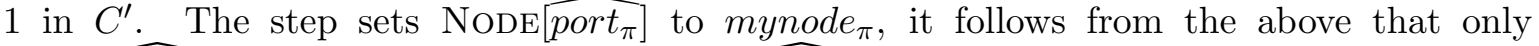
$\operatorname{NoDE}\left[\widehat{\text { port }_{\pi}}\right]=$ mynode $_{\pi}$ and $\forall q \in \mathcal{P}, q \neq \widehat{\text { port }_{\pi}} \Rightarrow \operatorname{NoDE}[q] \neq$ mynode $_{\pi}$ in $C^{\prime}$. Therefore, the condition holds in $C^{\prime}$.

Condition 4, By the same argument as for Condition 3 above, we have $\forall q \in \mathcal{P}, q \neq \widehat{\text { port }_{\pi}} \Rightarrow$ NODE $[q] \neq$ mynode $_{\pi}$ in $C^{\prime}$. Also, mynode $_{\pi}$.PRED $=$ NIL, therefore, from the definition of $\widehat{\operatorname{node}}_{\pi}$ it follows that the condition holds in $C^{\prime}$.

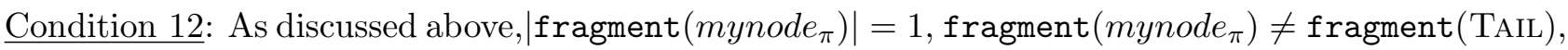
and mynode $_{\pi}=$ head $\left(\operatorname{fragment}\left(\right.\right.$ mynode $\left.\left._{\pi}\right)\right)$ in $C$, which continues to hold in $C^{\prime}$. Therefore, the condition holds in $C^{\prime}$.

13. $\pi$ executes Line 13 .

In $C, P C_{\pi}=13$ and $\widehat{P C_{\pi}}=\mathbf{1 3}$.

This step performs a FAS operation on the TAIL pointer so that TAIL now points to the same node as pointed by mynode ${ }_{\pi}$, and sets mypred $_{\pi}$ to the value held by TAIL in $C$. It updates $P C_{\pi}$ and $\widehat{P C_{\pi}}$ to $\mathbf{1 4}$.

Condition 9, Applying Condition 16 to $C$ we note that what holds true for TAIL in $C$, holds true for mypred $_{\pi}$ in $C^{\prime}$. Also applying Condition 8 to $C$ we note that mynode $_{\pi} \in \mathcal{N}^{\prime}$, mynode $_{\pi} \cdot \mathrm{PRED}=\mathrm{NIL}$, mynode $_{\pi}=\operatorname{head}\left(\operatorname{fragment}\left(\right.\right.$ mynode $\left.\left._{\pi}\right)\right), \mid \operatorname{fragment}\left(\right.$ mynode $\left._{\pi}\right) \mid=1$, fragment $\left(\right.$ mynode $\left._{\pi}\right) \neq \operatorname{fragment}($ TAIL $)$, mynode $e_{\pi}$.CS_SigNAL $=0$, and mynode $e_{\pi}$.NonNIL_SigNAL $=$ 0 in $C$. In $C^{\prime}$ it holds that $\operatorname{fragment}\left(\right.$ mynode $\left._{\pi}\right) \neq \operatorname{fragment}\left(\right.$ mypred $\left._{\pi}\right)$. It follows that the condition holds in $C^{\prime}$.

Condition 12, The truth value of the condition follows from the reasoning similar to Condition 9 as argued above.

Condition 16, By Condition 8, mynode $_{\pi} \in \mathcal{N}^{\prime}, \mid$ fragment $\left(\right.$ mynode $\left._{\pi}\right) \mid=1$, and mynode $_{\pi}$. PRED $=$ NIL in $C$. It follows from the same condition that tail $\left(\right.$ fragment $\left(\right.$ mynode $\left.\left._{\pi}\right)\right)=$ mynode $_{\pi}$. Since the step sets TAIL $=$ mynode $_{\pi}$ in $C^{\prime}$ and $\widehat{P C_{\pi}}=14$ in $C^{\prime}$, it follows that Condition 16 holds in $C^{\prime}$.

Condition 18, Suppose $|\mathcal{Q}|=0$ in $C$. By the condition, $\forall \pi^{\prime} \in \Pi, P C_{\pi^{\prime}} \in$ [11, 15, $\cup\{25\} \cup$ 28, 29 in $C$, which continues to hold in $C^{\prime}$. As argued above, mynode $e_{\pi}=\operatorname{head}\left(\operatorname{fragment}\left(\operatorname{mynode}_{\pi}\right)\right)$

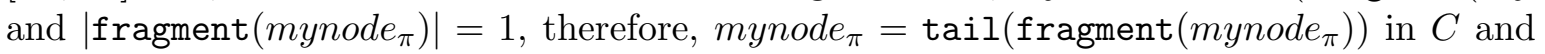
$C^{\prime}$. Since TAIL $=$ mynode $_{\pi}$ in $C^{\prime}$, the condition holds in $C^{\prime}$.

14. $\pi$ executes Line 14.

In $C, P C_{\pi}=14$ and $\widehat{P C_{\pi}}=\mathbf{1 4}$.

The step sets mynode $_{\pi} . \mathrm{PRED}=$ mypred $_{\pi}$ and updates $P C_{\pi}$ and $\widehat{P C_{\pi}}$ to $\mathbf{1 5}$.

Condition 1, By Condition 9, mypred $_{\pi} \in \mathcal{N}^{\prime}$ in $C$. Since the step sets mynode $_{\pi}$. Pred $=$ mypred $_{\pi}$, Node $\left[\right.$ port $\left._{\pi}\right]$.PRED $\in \mathcal{N}^{\prime}$ in $C^{\prime}$. Therefore, the condition holds in $C^{\prime}$.

Condition 3, By Condition 9, mypred $_{\pi}=\operatorname{tail}\left(\right.$ fragment (mypred $\left.\left._{\pi}\right)\right)$. Therefore, $\forall q \in$ $\mathcal{P}, \operatorname{NODE}[q]$. PRED $\neq$ mypred $_{\pi}$ in $C$. It follows that $\forall q \in \mathcal{P}, \widehat{\text { port }_{\pi}} \neq q \Rightarrow \operatorname{NoDE}[q] . \operatorname{PRED} \neq$ mypred $_{\pi}$ in $C^{\prime}$. Again from Condition 9 we observe the following about mypred $_{\pi}$. Either

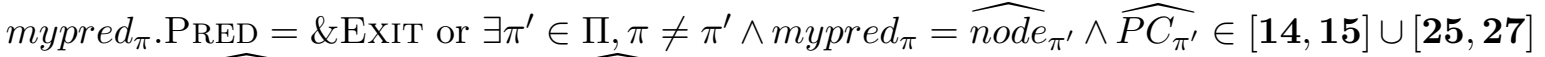
(i.e., Node $\left[\widehat{\text { port }_{\pi}}\right] . P R E D=\operatorname{Node}\left[\widehat{\text { port }_{\pi^{\prime}}}\right]$ ). Thus the condition holds in $C^{\prime}$.

Condition 44: By Condition 4. $\mid$ fragment $\left(\widehat{\operatorname{node}}_{\pi}\right) \mid=b_{1} \leq k$ and $\left|\operatorname{fragment}\left(\widehat{\operatorname{node}}_{\pi}\right)\right|=b_{2} \leq k$ in $C$. By Condition 7 , for a process $\pi^{\prime}$, it can not be the case that $\widehat{\operatorname{node}}_{\pi^{\prime}} \in \operatorname{fragment}\left(\widehat{\operatorname{node}}_{\pi}\right)$ and $\widehat{\operatorname{node}}_{\pi^{\prime}} \in$ fragment $\left(\right.$ mypred $\left._{\pi}\right)$ in $C$. Therefore, $b_{1}+b_{2} \leq k$ in $C$. It follows that in $C^{\prime}$ $\mid$ fragment $\left(\widehat{\operatorname{node}}_{\pi}\right) \mid=b_{1}+b_{2} \leq k$. Therefore, the condition holds in $C^{\prime}$.

Condition 7 fragment $\left(\right.$ mypred $\left._{\pi}\right)=\operatorname{fragment}\left(\widehat{\operatorname{rode}}_{\pi}\right)$ in $C^{\prime}$. Therefore, the condition holds 
in $C^{\prime}$ as it held and applied to fragment $\left(\right.$ mypred $\left._{\pi}\right)$ in $C$.

Condition 9. Applying the condition to fragment $\left(\widehat{\operatorname{node}}_{\pi}\right)$ in $C$, we get that $\forall \pi^{\prime} \in \Pi, \pi^{\prime} \neq$ $\widehat{\pi \wedge}_{\text {node }_{\pi^{\prime}}} \in \operatorname{fragment}\left(\widehat{\operatorname{node}}_{\pi}\right) \Rightarrow \widehat{P C_{\pi^{\prime}}} \in\{\mathbf{1 5}, \mathbf{2 5}\}$, which holds in $C^{\prime}$. We have $\widehat{P C_{\pi}}=15$ in $C^{\prime}$. Suppose there is a $\pi^{\prime \prime} \in \Pi, \pi^{\prime \prime} \neq \pi$ such that head(fragment $\left(\right.$ mypred $\left.\left._{\pi}\right)\right)=\widehat{\text { node }}_{\pi^{\prime \prime}}$ and $P C_{\pi^{\prime \prime}}=14$ in $C$. It follows that $\forall \pi^{\prime} \in \Pi, \pi^{\prime} \neq \pi^{\prime \prime} \wedge \widehat{\operatorname{node}}_{\pi^{\prime}} \in \operatorname{fragment}\left(\widehat{\operatorname{node}}_{\pi^{\prime \prime}}\right) \Rightarrow \widehat{P C_{\pi^{\prime}}} \in$ $\{15,25\}$ in $C^{\prime}$. Therefore, the condition holds for $\pi^{\prime \prime}$ and vacuously for other processes in $C^{\prime}$. Condition 13. Since $\widehat{\operatorname{node}}_{\pi}$. PrED $=$ mypred $_{\pi}$ in $C^{\prime}$ and invoking the Condition 9 on $C$ and mypred $_{\pi}$, it follows that the condition holds in $C^{\prime}$.

Condition 14, This condition holds by an argument similar to Condition 13 as argued above. Condition 15. Suppose head(fragment $\left(\right.$ mypred $\left.\left._{\pi}\right)\right)$.PRED $\in\{$ NIL,$\&$ CRASH $\}$ in $C$. It follows that $\exists \pi^{\prime} \in \Pi, \pi^{\prime} \neq \pi \wedge{\widehat{P C_{\pi^{\prime}}}}^{\prime}=\left[\mathbf{1 4} \wedge \widehat{\text { node }}_{\pi^{\prime}}=\operatorname{head}\left(\right.\right.$ fragment $\left.\left(\widehat{\operatorname{node}}_{\pi}\right)\right) \wedge\left(\forall \pi^{\prime \prime} \in \Pi,\left(\pi^{\prime \prime} \neq\right.\right.$ $\left.\left.\pi^{\prime} \wedge \widehat{\operatorname{node}}_{\pi^{\prime \prime}} \in \operatorname{fragment}\left(\widehat{\operatorname{node}}_{\pi}\right)\right) \Rightarrow{\widehat{P C_{\pi^{\prime \prime}}}} \in\{\mathbf{1 5}, \mathbf{2 5}\}\right)$ in $C^{\prime}$. Therefore, the condition holds in $C^{\prime}$.

Condition 19, If head (fragment $\left(\right.$ mypred $\left.\left._{\pi}\right)\right) . \mathrm{PRED} \in\{\mathrm{NIL}, \& \mathrm{CRASH}\}$ in $C$, it follows that $\pi \notin$ $\mathcal{Q}$ in $C^{\prime}$. Therefore, it is easy to see that the condition holds in $C^{\prime}$. If head $\left(\operatorname{fragment}\left(\right.\right.$ mypred $\left.\left._{\pi}\right)\right)$.PRED $\in$ $\{\& \mathrm{INCS}, \& \mathrm{ExIT}\}$ in $C$, it follows that $\pi \in \mathcal{Q}$ in $C^{\prime}$. We have two cases to consider, $|\mathcal{Q}|>0$ or $|\mathcal{Q}|=0$ in $C$. If $|\mathcal{Q}|>0$ in $C$, Conditions $19 \mathrm{~d}$ and $19 \mathrm{e}$ are the only ones affected. It is easy to see from the definition of a fragment that these conditions continue to hold in $C^{\prime}$, therefore, the whole condition would hold in $C^{\prime}$. Suppose $|\mathcal{Q}|=0$ and mypred $_{\pi}$.PRED $=\&$ ExIT in $C$. It follows that $\pi=\pi_{1}$ according to the ordering defined by the condition. Condition 19a holds in $C^{\prime}$ since $\widehat{P C_{\pi}}=15$ in $C^{\prime}$. Condition $19 \mathrm{~b}$ holds in $C^{\prime}$ since mypred $_{\pi}$.PRED $=\&$ EXIT in $C$ and $C^{\prime}$. Applying Condition 14 to mypred $_{\pi}$, and by the fact that mypred $_{\pi}$.PRED $=\&$ EXIT, it follows that Condition $19 \mathrm{c}$ holds in $C^{\prime}$. Conditions $19 \mathrm{~d}, 19 \mathrm{e}$, and $19 \mathrm{f}$ hold in $C^{\prime}$ by the definition of fragment mynode $\left._{\pi}\right)$ and the fact that mypred $_{\pi}$. PRED $=\&$ EXIT. Since $|\mathcal{Q}|=0$ in $C$, invoking Condition 18 on $C$ we see that Condition $19 \mathrm{~g}$ holds in $C^{\prime}$. Lastly, $\forall \pi^{\prime} \in \Pi, \pi^{\prime} \neq \pi \wedge \widehat{\operatorname{node}}_{\pi^{\prime}} \neq \mathrm{NIL} \wedge \widehat{\operatorname{node}}_{\pi^{\prime}}$.PRED $\in \mathcal{N}^{\prime}$ we have $\widehat{P C_{\pi^{\prime}}} \in\{$ 15, 25 , from Condition 1. Since $|\mathcal{Q}|=0$ in $C$, head $\left(\operatorname{fragment}\left(\widehat{\operatorname{node}}_{\pi^{\prime}}\right)\right)$.PRED $\in\{\mathrm{NIL}, \& \mathrm{CRASH}\}$ by definition of $\mathcal{Q}$. Therefore, by Condition $15, \widehat{\text { node }}_{\pi^{\prime}}$.CS_Signal $=0$ in $C$, which continues to hold in $C^{\prime}$.

15. $\pi$ executes Line 15 .

In $C, P C_{\pi}=15$ and $\widehat{P C_{\pi}}=15$.

The step executes mynode $e_{\pi}$.NonNIL_Signal.set () so that mynode $e_{\pi}$.NonNiL_SignaL $=1$ as a result of the step. It also updates $P C_{\pi}$ and $\widehat{P C_{\pi}}$ to 25 .

Condition 17. As discussed above, mynode $e_{\pi}$.NonNIL_SIGNAL $=1$ and $\widehat{P C_{\pi}}=25$ in $C^{\prime}$. Therefore, the condition holds in $C^{\prime}$.

16. $\pi$ executes Line 16.

In $C P C_{\pi}=16$ and $\widehat{P C_{\pi}} \in[\mathbf{1 3}, \mathbf{1 5} \cup[\mathbf{2 5}, \mathbf{2 9}$.

This step changes $P C_{\pi}$ to $\mathbf{1 7}$.

The step does not affect any condition, so the invariant continues to hold in $C^{\prime}$.

17. $\pi$ executes Line 17.

In $C P C_{\pi}=\mathbf{1 6}$ and $\widehat{P C_{\pi}} \in \mathbf{1 3}, \mathbf{1 5} \cup[\mathbf{2 5}, \mathbf{2 9}$.

This step sets mynode $_{\pi}$ to NoDE $\left[\right.$ port $\left._{\pi}\right]$ and changes $P C_{\pi}$ to $\mathbf{1 7}$

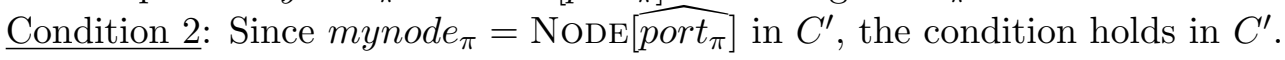

18 (a). $\pi$ executes Line 17 when $\widehat{P C_{\pi}} \in\{\mathbf{1 3}, \mathbf{1 4}\}$.

In $C, P C_{\pi}=18$ and $\widehat{P C_{\pi}} \in\left\{1 \mathbf{1 3},[14\}\right.$. By Condition $1, \widehat{\text { node }}_{\pi} \cdot \mathrm{PRED} \in\{\mathrm{NIL}, \& \mathrm{CrASH}\}$. 
This step checks if $\widehat{n o d e}_{\pi}$.PRED $=$ NIL and sets it to \&CRASH, if so. It then changes $P C_{\pi}$ to 19 .

Condition [1, By the step, $\widehat{\text { node }}_{\pi} \cdot \mathrm{PRED}=\&$ CRASH. Therefore, the condition holds in $C^{\prime}$.

Condition [10, Since $\widehat{P C_{\pi}} \in\{1 \mathbf{1 3}, 14\}, \widehat{\text { node }_{\pi}} \cdot \mathrm{PRED}=\& \mathrm{CRASH}$, and $P C_{\pi}=1 \mathbf{1 9}$, the condition holds in $C^{\prime}$.

Condition 11, The condition holds by the same argument as for Condition 10 above.

18 (b). $\pi$ executes Line $\mathbf{1 7}$ when $\widehat{P C_{\pi}} \notin\{13,14\}$.

In $C, P C_{\pi}=18$ and $\widehat{P C_{\pi}} \notin\{13,14\}$. By Condition 1, $\widehat{\text { node }}_{\pi}$.Pred $\notin\{\mathrm{NIL}, \& \mathrm{CrASH}\}$.

The if condition at Line $\mathbf{1 8}$ is not met, hence the step changes $P C_{\pi}$ to $\mathbf{1 9}$.

The step does not affect any condition, so the invariant continues to hold in $C^{\prime}$.

19. $\pi$ executes Line 19.

In $C P C_{\pi}=19$.

This step sets mypred $_{\pi}$ to NODE[ port $\left._{\pi}\right]$.PRED and changes $P C_{\pi}$ to 20.

Condition 2, Since mypred $_{\pi}=\operatorname{NoDE}\left[\right.$ port $\left._{\pi}\right]$.PRED in $C^{\prime}$, the condition holds in $C^{\prime}$.

20 (a). $\pi$ executes Line 20 when $\widehat{P C_{\pi}}=\mathbf{2 7}$.

In $C P C_{\pi}=20$ and $\widehat{P C}_{\pi}=27$. By Condition 1, $\widehat{\text { node }}_{\pi}$. PRED $=$ \&INCS. By Condition 2 , mypred $_{\pi}=\widehat{\operatorname{node}}_{\pi} \cdot$ PRED in $C$.

In this step the if condition is met, therefore, $\pi$ moves to the CS and updates $P C_{\pi}$ to 27 .

The step does not affect any condition, so the invariant continues to hold in $C^{\prime}$.

20 (b). $\pi$ executes Line 20 when $\widehat{P C_{\pi}} \neq \mathbf{2 7}$.

In $C P C_{\pi}=\mathbf{2 0}$ and $\widehat{P C_{\pi}} \neq \mathbf{2 7}$. By Condition $1, \widehat{\text { node }}_{\pi}$.PRED $\neq \&$ INCS. By Condition 2 , mypred $_{\pi}=\widehat{\operatorname{node}}_{\pi}$.PRED in $C$.

In this step the if condition is not met, therefore, $\pi$ updates $P C_{\pi}$ to $\mathbf{2 1}$.

The step does not affect any condition, so the invariant continues to hold in $C^{\prime}$.

21 (a). $\pi$ executes Line 21 when $\widehat{P C_{\pi}} \in\{\mathbf{2 8}, \mathbf{2 9}\}$.

In $C P C_{\pi}=21$ and $\widehat{P C_{\pi}} \in\left\{\left[\mathbf{2 8},[29\}\right.\right.$. By Condition 1, $\widehat{\text { node }}_{\pi} \cdot$ Pred $=\&$ Exit. By Condition 2, mypred $_{\pi}=\widehat{\text { node }}_{\pi}$. PRED in $C$.

In this step the if condition is met, therefore, $\pi$ updates $P C_{\pi}$ to $\mathbf{2 2}$

The step does not affect any condition, so the invariant continues to hold in $C^{\prime}$.

21 (b). $\pi$ executes Line 21 when $\widehat{P C_{\pi}} \notin\{2 \mathbf{2 8}, \mathbf{2 9}\}$.

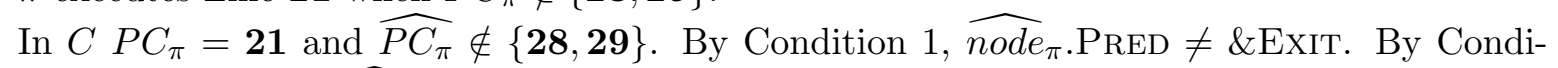
tion 2 . mypred $_{\pi}=\widehat{\text { node }}_{\pi}$.PRED in $C$.

In this step the if condition is not met, therefore, $\pi$ updates $P C_{\pi}$ to $\mathbf{2 3}$.

The step does not affect any condition, so the invariant continues to hold in $C^{\prime}$.

22. $\pi$ executes Line 22.

In $C P C_{\pi}=\mathbf{2 2}$ and $\widehat{P C_{\pi}} \in\{\mathbf{2 8}, 2 \mathbf{2 9}\}$.

$\pi$ sets $\widehat{P C_{\pi}}=\mathbf{2 8}$, then executes Lines $\mathbf{2 8}$ and $\mathbf{2 9}$ as part of the Try section, and then changes $P C_{\pi}$ to $\mathbf{1 0}$ and $\widehat{P C_{\pi}}$ to $\mathbf{1 1}$

For the correctness of the invariant, refer to the induction steps for Lines $\mathbf{2 8}$ and $\mathbf{2 9}$, since the execution of the step is same as executing the two lines and then executing a "go to Line $\mathbf{1 0}$ '.

23. $\pi$ executes Line 23.

In $C P C_{\pi}=$ 23. By Condition $6, \widehat{P C_{\pi}} \in[\mathbf{1 3}, 15] \cup[\mathbf{2 5}, \mathbf{2 6}$. 
The step executes mynode $e_{\pi}$.NonNIL_Signal.set () so that mynode $e_{\pi}$.NonNIL_SignAL $=1$ as a result of the step. It also updates $P C_{\pi}$ to $\mathbf{2 4}$.

Condition 17, As discussed above, mynode $_{\pi}$.NonNil_Signal $=1$ and $P C_{\pi}=\mathbf{2 4}$ in $C^{\prime}$. Therefore, the condition holds in $C^{\prime}$.

24. $\pi$ executes Line 24.

In $C P C_{\pi}=24$, By Condition 6, $\widehat{P C_{\pi}} \in[\mathbf{1 3}, 15] \cup[25,26]$ in $C$.

The step executes the Try section of RLOCK in order to access the Critical Section of RLock starting at Line 30. Since the RLOCK is assumed to be satisfying Starvation Freedom, $\pi$ reaches Line 30 eventually. Hence, the step changes $P C_{\pi}$ to $\mathbf{3 0}$. Note, we can use Golab and Ramaraju's [7] read-write based recoverable extension of Yang and Anderson's lock (see Section 3.2 in [7]) as RLOCK for this purpose.

The step does not affect any condition, so the invariant continues to hold in $C^{\prime}$.

25. $\pi$ executes Line 25

In $C, P C_{\pi}=25$ and $\widehat{P C}_{\pi}=25$. We have $\widehat{\operatorname{node}}_{\pi} \neq$ NIL and $\widehat{\operatorname{node}}_{\pi}$. PRED $\in \mathcal{N}^{\prime}$ by Condition 1 in $C$. By Condition 14 , either $\widehat{\text { node }}_{\pi}$.PRED.CS_SignAL $=1$, or $\exists \pi^{\prime} \in \Pi, \pi \neq \pi^{\prime} \wedge \widehat{\text { node }}_{\pi^{\prime}}=$ $\widehat{\operatorname{node}}_{\pi} . \mathrm{PRED} \wedge \widehat{P C_{\pi^{\prime}}} \in\{\mathbf{1 4}, \mathbf{1 5}\} \cup[\mathbf{2 5}, \mathbf{2 8}$ in $C$.

The step executes mypred $_{\pi}$.CS_SignAL.wait() so that the procedure call returns when mypred . $_{\pi}$.CS_SIGNAL 1. The step also updates $P C_{\pi}$ and $\widehat{P C_{\pi}}$ to $\mathbf{2 6}$ when it returns from the procedure call.

Condition 19. Suppose $\widehat{\operatorname{node}}_{\pi}$.Pred.CS_SignAl $=1$ in $C$. By Condition $19 \mathrm{~h} \pi=\pi_{1}$ in $C$. It follows that the condition continues to hold in $C^{\prime}$ as it held in $C$. Therefore, assume $\widehat{n o d e}_{\pi}$.PRED.CS_Signal $\neq 1$ in $C$. Since mypred $_{\pi}$.CS_Signal.wait () returns and the step completes, by the specification of the Signal object, mypred $_{\pi}$. CS_SignAL $=1$ in $C^{\prime}$. By Condition 5. mypred $_{\pi}$.PRED $=\&$ ExIT in $C^{\prime}$. Therefore, head $\left(\right.$ fragment $\left.\left(\widehat{\operatorname{rode}}_{\pi}\right)\right) \cdot$ PRED $=$ \&Exit in $C^{\prime}$. It follows that $\pi \in \mathcal{Q}$ in $C^{\prime}$ by the definition of $\mathcal{Q}$. By Condition 7 , $\forall \pi^{\prime} \in \Pi,\left(\pi \neq \pi^{\prime} \wedge \operatorname{head}\left(\right.\right.$ fragment $\left.\left(\widehat{\operatorname{node}}_{\pi^{\prime}}\right)\right) . \mathrm{PRED}=\& \operatorname{ExIT} \wedge \widehat{P C_{\pi^{\prime}}} \notin[\mathbf{2 8},[\mathbf{2 9}]) \Rightarrow \widehat{\operatorname{node}}_{\pi^{\prime}} \in$ fragment $\left(\widehat{\operatorname{node}}_{\pi}\right)$. We now proceed to prove that Condition 19 holds in $C^{\prime}$ as follows. We have mypred $_{\pi} . \mathrm{PRED}=\&$ EXIT and mypred $_{\pi}$. CS_SIGNAL $=1$ in $C^{\prime}$. Therefore, $\forall \pi^{\prime} \in \Pi, \widehat{\text { node }}_{\pi^{\prime}}=$ mypred $_{\pi} \Rightarrow \widehat{P C_{\pi^{\prime}}}=29$ in $C^{\prime}$ by Condition 5. Since $\widehat{\text { node }}_{\pi}$.PRED.PRED $=$ \&ExIT, Condition $19 \mathrm{f}$ holds and it follows that $\pi=\pi_{1}$. Since $\widehat{P C_{\pi}}=\mathbf{2 6}$, Condition 19a holds in $C^{\prime}$. We also note from the above that Conditions $19 \mathrm{~b}$ and $19 \mathrm{c}$ hold in $C^{\prime}$. By the definition of fragment $\left(\widehat{\operatorname{node}}_{\pi}\right)$ and Condition 7 it follows that Conditions $19 \mathrm{~d}$ and $19 \mathrm{e}$ hold. For any process $\pi^{\prime}$, if $\widehat{\operatorname{node}}_{\pi^{\prime}}$.PRED $=\&$ ExIT, then by Condition 1 , $\widehat{P C_{\pi^{\prime}}} \in\{[\mathbf{2 8},[29\}$. If $\widehat{\operatorname{node}}_{\pi^{\prime}} \neq \operatorname{head}\left(\right.$ fragment $\left.\left(\widehat{\text { node }}_{\pi^{\prime}}\right)\right)$, then by Condition $7, \widehat{P C_{\pi^{\prime}}} \in\{\mathbf{1 5}$, 25 $\}$. If $\widehat{\operatorname{node}}_{\pi^{\prime}}$.PRED $\in$ $\{\mathrm{NIL}, \& \mathrm{CRASH}\}$, then by Condition $1, \widehat{P C_{\pi^{\prime}}} \in\{1 \mathbf{1 3}, 14\}$. By Condition 7 there is only one fragment whose head node has its PRED pointer set to \&EXIT. It follows that Condition $19 \mathrm{~g}$ holds from the above. From Conditions 5, 9, 14, and 15 it follows that Condition 19h holds in $C^{\prime}$. Therefore, the entire condition holds in $C^{\prime}$.

26. $\pi$ executes Line 26.

In $C P C_{\pi}=\mathbf{2 6}$.

The step sets $\widehat{\operatorname{node}}_{\pi} \cdot \mathrm{PRED}=\& \mathrm{INCS}$, updates $P C_{\pi}$ and $\widehat{P C_{\pi}}$ to $\mathbf{2 7}$, and goes to the CS.

Condition 1. As argued above, $\widehat{\operatorname{node}}_{\pi} \cdot \mathrm{PRED}=\& \mathrm{INCS}$ and $\widehat{P C_{\pi}}=\mathbf{2 7}$ in $C^{\prime}$. Therefore, the condition holds in $C^{\prime}$.

Condition 98. Suppose there is a $\pi^{\prime \prime} \in \Pi, \pi^{\prime \prime} \neq \pi$ such that mypred $_{\pi^{\prime \prime}}=\widehat{\operatorname{node}}_{\pi}$ in $C$. It follows that $\widehat{\operatorname{node}}_{\pi}$. PRED $=\&$ INCS and $\widehat{P C_{\pi}}=27$ in $C^{\prime}$. Therefore, the condition holds for $\pi^{\prime \prime}$ and 
vacuously for other processes in $C^{\prime}$.

27. $\pi$ executes Line 27.

In $C P C_{\pi}=27$.

The step sets $\overline{\text { node }}_{\pi}$. PRED $=\&$ ExIT, and updates $P C_{\pi}$ and $\widehat{P C_{\pi}}$ to $\mathbf{2 8}$.

Condition 1. As argued above, $\widehat{\operatorname{node}}_{\pi} \cdot \mathrm{PRED}=\&$ ExIT and $\widehat{P C_{\pi}}=\mathbf{2 8}$ in $C^{\prime}$. Therefore, the condition holds in $C^{\prime}$.

Condition 9. Suppose there is a $\pi^{\prime \prime} \in \Pi, \pi^{\prime \prime} \neq \pi$ such that mypred $_{\pi^{\prime \prime}}=\widehat{\operatorname{node}}_{\pi}$ in $C$. It follows that $\widehat{\operatorname{node}}_{\pi} \cdot$ PRED $=\&$ EXIT and $\widehat{P C_{\pi}}=\mathbf{2 8}$ in $C^{\prime}$. Therefore, the condition holds for $\pi^{\prime \prime}$ and vacuously for other processes in $C^{\prime}$.

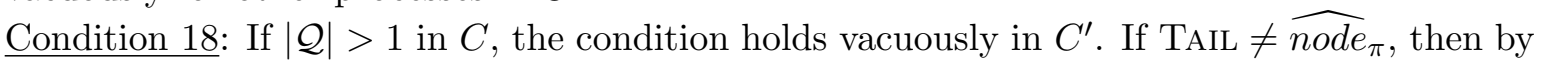
Condition 7 and 16 , the condition holds in $C^{\prime}$. Otherwise, suppose $|\mathcal{Q}|=1$ and TAIL $=\widehat{\text { node }}_{\pi}$ in $C$. TAil.Pred $=\&$ Exit in $C^{\prime}$ by the step. Therefore, the condition holds in $C^{\prime}$.

Condition 19. Applying the condition to $\pi$ in $C, \pi=\pi_{1}$ according to the ordering of the condition. If $|\mathcal{Q}|=1$, the condition holds vacuously in $C^{\prime}$. Therefore, suppose $|\mathcal{Q}|>1$ in $C$. There is a process $\pi^{\prime} \in \Pi$ such that $\pi^{\prime}=\pi_{2}$ according to the ordering and mypred $_{\pi^{\prime}}=\widehat{\text { node }}_{\pi}$ in $C$. By Condition $19 \mathrm{~d}, \widehat{P C_{\pi^{\prime}}} \in\{\mathbf{1 5}, 25\}$ in $C$ which continues to hold in $C^{\prime}$. Therefore, it follows that Conditions $19 \mathrm{a}, 19 \mathrm{~b}$, and $19 \mathrm{c}$ hold for $\pi^{\prime}$ in $C^{\prime}$. It is easy to see that the rest of the sub-conditions hold in $C^{\prime}$ as a result of the step. Therefore, the condition holds in $C^{\prime}$.

28. $\pi$ executes Line 28 .

In $C P C_{\pi}=28$ and $\widehat{P C_{\pi}}=\mathbf{2 8}$

The step executes mynode $e_{\pi}$.CS_SignAL.set () so that mynode $e_{\pi}$.CS_SIGNAL $=1$ as a result of the step. It also updates $P C_{\pi}$ and $\widehat{P C_{\pi}}$ to 29 .

Condition [17. As discussed above, mynode ${ }_{\pi}$.CS_SIGNAL $=1$ and $\widehat{P C_{\pi}}=29$ in $C^{\prime}$. Therefore, the condition holds in $C^{\prime}$.

29. $\pi$ executes Line 29.

In $C P C_{\pi}=\mathbf{2 9}$ and $\widehat{P C_{\pi}}=\mathbf{2 9}$.

The step sets NoDE $\left[\right.$ port $\left._{\pi}\right]$ to NIL, sets $P C_{\pi}$ to $\mathbf{1 0}$, and $\widehat{P C_{\pi}}$ to $\mathbf{1 1}$.

Condition 1, As argued above, NoDE $\left.\widehat{\text { port }_{\pi}}\right]=\mathrm{NIL}$ and $\widehat{P C_{\pi}}=11$ in $C^{\prime}$. Therefore, the condition holds in $C^{\prime}$.

Condition 5: By Condition 3 implies that $\forall \pi^{\prime} \in \Pi, \pi^{\prime} \neq \pi \Rightarrow \widehat{\operatorname{node}}_{\pi^{\prime}} \neq \widehat{\operatorname{node}}_{\pi}$ in $C$. By Condition 1. $\widehat{\operatorname{node}}_{\pi} \cdot$ Pred $=\&$ EXIT in $C$ which holds in $C^{\prime}$. By Condition 17, $\widehat{\text { node }}_{\pi}$. CS $\_$SignAL $=1$ and $\widehat{\operatorname{node}}_{\pi}$. NONNIL_SignAL $=1$ in $C$, which holds in $C^{\prime}$. Since $\widehat{\operatorname{node}}_{\pi}=$ NIL in $C^{\prime}$, it follows that for the QNode pointed to by $\widehat{\operatorname{node}}_{\pi}$ in $C$ the condition holds in $C^{\prime}$.

Condition 9. Suppose there is a $\pi^{\prime \prime} \in \Pi, \pi^{\prime \prime} \neq \pi$ such that mypred $_{\pi}=\widehat{\operatorname{node}}_{\pi^{\prime \prime}}$ and $P C_{\pi^{\prime \prime}}=\mathbf{1 4}$ in $C$. It follows that $\forall p^{\prime} \in \mathcal{P}, \operatorname{NoDE}\left[p^{\prime}\right] \neq$ mypred $_{\pi}$ in $C^{\prime}$. Therefore, the condition holds for $\pi^{\prime \prime}$ and vacuously for other processes in $C^{\prime}$.

30 (a). $\pi$ executes Line 30 when $\widehat{P C_{\pi}} \in\{1 \mathbf{1 3}, 14\}$.

In $C, P C_{\pi}=30$ and $\widehat{P C_{\pi}} \in\{13,14\}$. By Condition $10, \widehat{\text { node }}_{\pi}$.Pred $=\&$ Crash. By Condition 2, mypred $_{\pi}=\widehat{\text { node }}_{\pi}$.PRED in $C$.

The if condition at Line 30 is not met since $\widehat{\operatorname{node}}_{\pi}$. PRED $=\&$ CRASH, therefore, $P C_{\pi}$ changes to 31 .

Condition [6. Since $P C_{\pi}=31$ and $\widehat{P C_{\pi}} \in\{13,14\}$ in $C^{\prime}$, the condition is satisfied.

Condition 20, Suppose head(fragment(TAIL)).PrEd $\in\{\&$ InCS, \&EXIT $\}$ in $C$. We have $\widehat{\operatorname{node}}_{\pi} \cdot \operatorname{PRED}=\& \mathrm{CrASH}$, i.e., head $\left(\right.$ fragment $\left.\left(\widehat{\operatorname{node}}_{\pi}\right)\right) \cdot \mathrm{PRED}=\& \mathrm{CRASH}$. It follows that 
fragment $\left(\widehat{\operatorname{node}}_{\pi}\right) \neq$ fragment(TAIL) in $C$, which holds in $C^{\prime}$. Therefore, the condition holds in $C^{\prime}$.

30 (b). $\pi$ executes Line 30 when $\widehat{P C_{\pi}} \notin\{13,14\}$.

In $C, P C_{\pi}=\widehat{30}$ and $\widehat{P C_{\pi}} \notin\{\mathbf{1 3}, \mathbf{1 4}\}$. By Condition $6, \widehat{P C_{\pi}} \in\{\mathbf{1 5}\} \cup\{\mathbf{2 5}, \mathbf{2 6}\}$. By Condition 10, $\widehat{\text { node }}_{\pi}$. Pred $\in \mathcal{N}^{\prime}$. By Condition 2 , mypred ${ }_{\pi}=\widehat{\operatorname{node}}_{\pi}$. PRED in $C$.

The if condition at Line $\mathbf{3 0}$ is met since $\widehat{\operatorname{node}}_{\pi}$. PRED $\neq \& \mathrm{CRASH}$, therefore, $\pi$ executes the Exit section of RLOCK. $\pi$ then changes $P C_{\pi}$ and $\widehat{P C_{\pi}}$ to 25 .

Condition 17. Applying Condition 17 to $C$, we have $\widehat{n o d e}_{\pi}$. NonNIL_Signal $=1$ since $P C_{\pi}=$ 30 in $C$. Therefore, the condition holds in $C^{\prime}$.

31. $\pi$ executes Line 31.

In $C P C_{\pi}=31$.

The step initializes tail $_{\pi}$ to TAIL, the set $V_{\pi}$ and $E_{\pi}$ as empty sets, tailpath $h_{\pi}$ to NIL, and headpath $_{\pi}$ to NIL. Since the invariant requires that $i_{\pi}$ be between $[0, k]$ when $P C_{\pi}=\mathbf{3 2}$, we assume that the step implicitly initializes $i_{\pi}$ to 0 , although not noted in the code. Finally, the step sets $P C_{\pi}$ to $\mathbf{3 2}$.

Condition 21, Follows immediately from the description of the step above.

Condition 22, This condition follows immediately from Condition 16.

Condition 23. Since $\left(V_{\pi}, E_{\pi}\right)$ are initialized to be empty sets, the condition follows.

Condition 24, Consider the fragments formed from the nodes pointed to by the cells in the Node array. If all the fragments have the PRED pointer of their head node to be in $\{\mathrm{NIL}, \& \mathrm{CrASH}\}$, then by definition of $\mathcal{Q}$ it is an empty set. Hence, Condition $24 \mathrm{~d}$ holds. Otherwise, there is a fragment whose head node has its PRED pointer to be one of $\{\&$ InCS, \&EXIT $\}$. It follows that Condition $24 \mathrm{c}$ holds.

Condition 25. By Condition 12 it follows that $\forall \widehat{\operatorname{node}}_{\pi^{\prime}} \in \operatorname{fragment}\left(\widehat{\operatorname{node}}_{\pi}\right), \exists i \in[0, k-$ 1], NoDE $[i]=\widehat{\operatorname{node}}_{\pi^{\prime}}$. Therefore, the condition holds in $C^{\prime}$.

Condition 26. Since $\left(V_{\pi}, E_{\pi}\right)$ is an empty set in $C^{\prime}$, the condition holds vacuously.

Condition 27, All the conditions holds vacuously since the graph is empty and $i_{\pi}=0$.

Condition 28, Suppose head $\left(\right.$ fragment $\left(\right.$ tail $\left.\left._{\pi}\right)\right)$.PRED $\in\{\&$ INCS, \&EXIT $\}$. Since $\widehat{\operatorname{node}}_{\pi} \cdot$ PRED $=$ \&CRASH in $C^{\prime}$, it follows that the condition holds in $C^{\prime}$.

Condition 29. Immediate from the description of the step above.

32 (a). $\pi$ executes Line 32 when $i_{\pi}<k$.

In $C, P C_{\pi}=\mathbf{3 2}$ and $i_{\pi}<k$.

In this step the correctness condition of the for loop (i.e., $i_{\pi} \in[0, k-1]$ ) evaluates to true and $P C_{\pi}$ is updated to $\mathbf{3 3}$.

Since no shared variables are changed and no condition of the invariant is affected by the step, all the conditions continue to hold in $C$ as they held in $C^{\prime}$.

32 (b). $\pi$ executes Line 32 when $i_{\pi}=k$.

In $C, P C_{\pi}=32$ and $i_{\pi}=k$.

In this step the correctness condition of the for loop (i.e., $i_{\pi} \in[0, k-1]$ ) evaluates to false and $P C_{\pi}$ is updated to $\mathbf{3 9}$.

Condition 28, From Condition 22 it follows that either tail $_{\pi} \in V_{\pi} \vee \operatorname{tail}_{\pi}$. PRED $=\&$ EXIT in $C^{\prime}$. In either case the condition holds in $C^{\prime}$.

Condition 32, This follows immediately from Conditions 23, 25, 27 and the definition of fragment. 
Condition 34 Follows immediately from Condition 24 and the fact that $i_{\pi}=k$.

Condition 35. Follows from Conditions 23, 24, 26, 27, the definition of fragment, and the fact that $\widehat{\operatorname{rode}}_{\pi}$. PRED $=$ \&CRASH.

33. $\pi$ executes Line 33 .

In $C, P C_{\pi}=33$. By Condition 21, $i_{\pi} \in[0, k-1]$.

In this step, $\pi$ sets cur $_{\pi}$ to $\operatorname{NoDE}\left[i_{\pi}\right]$. It then updates $P C_{\pi}$ to 35 .

Condition 30, NODE $\left[i_{\pi}\right]$ either has the value NIL or it does not. If it is the first case, we are done. In the second the condition follows from Condition 3 .

34 (a). $\pi$ executes Line 34 when cur $_{\pi}=$ NIL.

In $C, P C_{\pi}=34$, and cur $_{\pi}=$ NIL.

Since the if is met, $\pi$ is required to break the current iteration of the loop and start with its next iteration. Therefore, $\pi$ increments $i_{\pi}$ by 1 and changes $P C_{\pi}$ to $\mathbf{3 2}$.

Since no shared variables are changed and no condition of the invariant is affected by the step, all the conditions continue to hold in $C$ as they held in $C^{\prime}$.

34 (b). $\pi$ executes Line 34 when cur $_{\pi} \neq$ NIL.

In $C, P C_{\pi}=34$ and cur $_{\pi} \neq$ NIL.

Since the if is not met, $\pi$ changes $P C_{\pi}$ to 35 .

Condition 30, The condition holds in $C^{\prime}$ as it held in $C$.

35. $\pi$ executes Line 35 .

In $C P C_{\pi}=\mathbf{3 5}$.

The step executes cur $_{\pi}$.NonNiL_Signal.wait() so that the procedure call returns when cur $_{\pi}$.NONNil_Signal $=1$. The step also updates $P C_{\pi}$ to $\mathbf{3 6}$ when it returns from the procedure call.

Condition 30, Since $c u r_{\pi}$.NonNil_Signal $=1$ as a result of the step, by Condition 5 , cur $_{\pi}$. PrED $\in\{\&$ CRASh, \&InCS, \&Exit $\}$ in $C^{\prime}$. Therefore, the condition holds in $C^{\prime}$.

36. $\pi$ executes Line 36 .

In $C P C_{\pi}=\mathbf{3 6}$.

The step sets curpred $_{\pi}$ to cur $_{\pi}$.PRED and updates $P C_{\pi}$ to $\mathbf{3 7}$

Since no shared variables are changed and no condition of the invariant is affected by the step, all the conditions continue to hold in $C$ as they held in $C^{\prime}$.

37 (a). $\pi$ executes Line 37 when curpred $_{\pi} \in\{\&$ CRASH, \&INCS, \&EXIT $\}$.

In $C, P C_{\pi}=\mathbf{3 7}$ and curpred $_{\pi} \in\{\& \mathrm{CRASH}, \& \mathrm{INCS}, \& \mathrm{EXIT}\}$.

The if condition at Line 37 is met, therefore, the step adds cur $_{\pi}$ to the set $V_{\pi}$. It then increments $i_{\pi}$ by 1 and updates $P C_{\pi}$ to $\mathbf{3 2}$.

Condition 23. Since the step adds only a vertex to the graph, the condition remains unaffected by the step.

Condition 24, If cur $_{\pi}$.PRED $=$ \&INCS, then Condition 24b is satisfied by the addition of $\mathrm{cur}_{\pi}$ to $V_{\pi}$. Otherwise, the condition holds as it held in $C^{\prime}$.

Condition 26. If cur $_{\pi}$.PRED $=\&$ CRASH, then the condition holds vacuously. Otherwise, cur $_{\pi}$. PRED $\in\{\&$ INCS, \&EXIT $\}$ and it follows that the condition holds in $C^{\prime}$.

Condition 27, All sub-conditions are easy to argue, hence it follows that the condition holds in $C^{\prime}$.

37 (b). $\pi$ executes Line 37 when curpred $_{\pi} \notin\{\&$ CRASH, \&InCS, \&EXIT $\}$.

In $C, P C_{\pi}=37$ and curpred $_{\pi} \notin\{\& \mathrm{CRASH}, \&$ INCS, \&EXIT $\}$. 
The if condition at Line $\mathbf{3 7}$ is not met, therefore, $\pi$ updates $P C_{\pi}$ to $\mathbf{3 8}$.

Condition 28. Since curpred $_{\pi} \notin\{\& \mathrm{CRASH}, \& \mathrm{INCS}, \& \mathrm{EXIT}\}$, curpred $_{\pi}$ was initialized from $\operatorname{cur}_{\pi}$.PRED at Line 36. It follows that curpred $_{\pi} \in \mathcal{N}^{\prime}$. Therefore the condition holds in $C^{\prime}$.

38. $\pi$ executes Line 38.

In $C P C_{\pi}=\mathbf{3 8}$.

The step adds the elements cur $_{\pi}$ and curpred $_{\pi}$ to the set $V_{\pi}$ and the edge $\left(\right.$ cur $_{\pi}$, curpred $\left._{\pi}\right)$ to the set $E_{\pi}$. It then increments $i_{\pi}$ by 1 and updates $P C_{\pi}$ to $\mathbf{3 2}$.

Condition 23, If cur $_{\pi}$.PRED $\notin V_{\pi}$ in $C$, then the condition holds in $C^{\prime}$. Hence, assume cur $_{\pi}$.PRED $\in V_{\pi}$ in $C$ (note, cur $_{\pi}$.PRED $=$ curpred $_{\pi}$ in $C$ ). We have to argue that after the addition of the edge $\left(\right.$ cur $_{\pi}$, curpred $\left._{\pi}\right)$ in $E_{\pi}$, the graph $\left(V_{\pi}, E_{\pi}\right)$ still remains directed and acyclic and the maximal paths in it remain disjoint in $C^{\prime}$. During the configuration $C$, let $\sigma_{1}$ be the path in the graph $\left(V_{\pi}, E_{\pi}\right)$ containing cur $_{\pi}$ and $\sigma_{2}$ be the path containing curpred $_{\pi}$.

If $\sigma_{1} \neq \sigma_{2}$, then the graph $\left(V_{\pi}, E_{\pi}\right)$ continues to be directed and acyclic in $C^{\prime}$. We argue that the maximal paths are disjoint as follows. Suppose for a contradiction that after adding the edge $\left(\right.$ cur $_{\pi}$, curpred $\left._{\pi}\right)$ there are two maximal paths $\sigma$ and $\sigma^{\prime}$ that are not disjoint. It follows that this situation arises due to the addition of the edge $\left(\right.$ cur $_{\pi}$, curpred $\left._{\pi}\right)$, hence $\sigma$ and $\sigma^{\prime}$ either share cur $_{\pi}$ or curpred $_{\pi}$. Suppose they share $c u r_{\pi}$ as a common vertex. In $C$ there is an edge $\left(\operatorname{cur}_{\pi}, u\right) \in E_{\pi}$ (and therefore in the path $\sigma_{1}$ ) such that $u \neq$ curpred $_{\pi}$. Applying Condition 27i to $C, c_{\pi}$.PRED $=u$ or $c u r_{\pi}$.PRED $\in\{\&$ INCS, \&EXIT $\}$ which is impossible since cur $_{\pi} . \mathrm{PRED}=$ curpred $_{\pi}$. Hence, assume that they share curpred $_{\pi}$ as a common vertex. It follows that there is an edge $\left(v\right.$, curpred $\left._{\pi}\right) \in E_{\pi}$ appearing in the path $\sigma_{2}$ in the configuration $C$. By Condition 27c $\exists i^{\prime} \in\left[0, i_{\pi}-1\right], v=\operatorname{Node}\left[i^{\prime}\right]$ or $v . \operatorname{PrED}=\& \operatorname{ExiT} \wedge \forall p^{\prime} \in \mathcal{P}, \operatorname{Node}\left[p^{\prime}\right] \neq$ $v$. If $\exists i^{\prime} \in\left[0, i_{\pi}-1\right], v=\operatorname{Node}\left[i^{\prime}\right]$, then Node $\left[i^{\prime}\right]$.Pred $=\operatorname{Node}\left[i_{\pi}\right]$.Pred, which contradicts Condition 3. Otherwise, by Condition 27h. $\forall i^{\prime} \in[0, k-1]$, Node $\left[i^{\prime}\right]$.PRED $\neq$ curpred $_{\pi}$, a

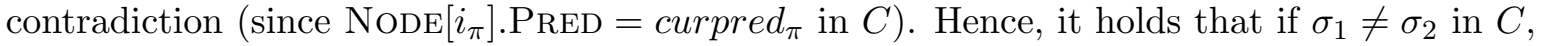
the maximal paths are disjoint in the graph in $C^{\prime}$.

Otherwise, $\sigma_{1}=\sigma_{2}$. It follows that $\operatorname{start}\left(\sigma_{1}\right)=\operatorname{start}\left(\sigma_{2}\right)=\operatorname{curpred}_{\pi}$ and end $\left(\sigma_{1}\right)=$ end $\left(\sigma_{2}\right)=\operatorname{cur}_{\pi}$ (i.e., there is a path from curpred $_{\pi}$ to cur $_{\pi}$ ) in $C$. Applying Condition 27i inductively we see that curpred $_{\pi}$. PRED $\neq \&$ EXIT, otherwise it would imply cur $_{\pi}$. PRED $=$ $\&$ ExIT. It follows by the contrapositive of Condition $27 \mathrm{c}$ that there is a distinct $i^{\prime} \in\left[0, i_{\pi}-1\right]$ for every vertex $w$ in the path $\sigma_{1}$ such that $\operatorname{Node}\left[i^{\prime}\right]=w$ in $C$. That is, every vertex $w$ in the path $\sigma_{1}$ is also a node $\widehat{\operatorname{node}}_{\pi^{\prime}}$ for some $\pi^{\prime} \in \Pi$. However, since cur $_{\pi}$.PRED $\notin$ $\{$ NIL, \&CRASH, \&INCS, \&ExIT $\}$ (because there is a cycle with the presence of $\sigma_{1}$ and the pointer $\mathrm{cur}_{\pi}$.PRED), we have a contradiction to Condition 4 since there is no $b \in \mathbb{N}$ for which the condition is satisfied. Therefore, $\sigma_{1} \neq \sigma_{2}$ in $C$.

From this argument it follows that the condition holds in $C^{\prime}$.

Condition 24: If Condition 24a holds in $C$, it continues to hold in $C^{\prime}$ and therefore the condition is satisfied. Similarly for Condition $24 \mathrm{~b}$, because cur $_{\pi}$. PRED $\neq$ \&EXIT and if $\operatorname{cur}_{\pi}=\operatorname{end}(\sigma)$ for some maximal path which satisfied the condition, then it continues to satisfy the condition in $C^{\prime}$. If $i_{\pi}<k-1$ and the condition held in $C$ due to Condition 24c, then it continues to hold in $C^{\prime}$ for the new value of $i_{\pi}$. If $i_{\pi}=k-1$ and the condition held in $C$ due to Condition 24c, it follows that NoDE $[k-1]$.PRED $\neq \&$ Exit (by assumption above) and Node $[k-1]$.PrEd.PrEd $\in\{\&$ InCS, $\&$ Exit $\}$. Therefore, by the step (Node $[k-$ 1], Node[ $k-1]$.PRED) is added as an edge in the graph and we have a path that satisfies Condition 24b in $C^{\prime}$. If Condition 24d holds in $C$, then it holds in $C^{\prime}$ as well.

Condition 25, This condition holds by the definition of fragment and since the edge gets 
added to the graph.

Condition 26. It is easy to see that the second part of the condition holds because one of the nodes among $v$ and $v^{\prime}$ was used up to enter the CS and hence even though the path runs through that node in the graph, the fragment is cut. Therefore, we argue the first part as follows. Suppose there is a $i<i_{\pi}$ such that $\operatorname{NoDE}[i]=\widehat{\text { node }}$ for a $\widehat{\text { node }} \in \operatorname{fragment}(v)$. In a previous iteration the node was added in the graph, and if $\widehat{\text { node }}$.PRED was an actual node, then it also got added to the graph along with an edge between them. Therefore, the condition holds in $C^{\prime}$.

Condition 27, As argued above, cur $_{\pi}$. PRED $\in \mathcal{N}^{\prime}$ (i.e., curpred $_{\pi} \in \mathcal{N}^{\prime}$ ), it follows that $\forall v \in$ $V_{\pi}, v \in \mathcal{N}^{\prime}$ in $C^{\prime}$. It is easy to see that the rest part of Condition 27a holds. Condition 27i holds because if cur $_{\pi}$. PRED $\in\left\{\& \mathrm{INCS}_{\mathrm{N}} \& \mathrm{ExIT}\right\}$, then the owner of curpred $_{\pi}$ already completed Line 28 to let the owner of $\operatorname{cur}_{\pi}$ into CS. Condition 27h holds from Condition 3 . It is easy to see that the remaining sub-conditions hold in $C^{\prime}$.

39. $\pi$ executes Line 39.

In $C, P C_{\pi}=39$.

The step computes the maximal paths in the graph $\left(V_{\pi}, E_{\pi}\right)$ and the set Paths $s_{\pi}$ contains every such maximal path. The step then sets $P C_{\pi}$ to 40 .

Condition 37, If there is a maximal path $\sigma$ in $\left(V_{\pi}, E_{\pi}\right)$ such that end $(\sigma)$.PRED $\in\{\&$ InCS, \&ExIT $\}$ and $\operatorname{start}(\sigma) . \operatorname{PRED} \neq \&$ ExIT, then the condition holds vacuously. Otherwise there is no maximal path $\sigma$ for which end $(\sigma)$.PRED $\in\{\& \operatorname{INCS}, \&$ ExIT $\}$ and $\operatorname{start}(\sigma)$.PRED $\neq \&$ ExIT. By Condition 34, head (fragment $\left(\right.$ tail $\left.\left._{\pi}\right)\right)$.PrEd $\in\{\& \operatorname{INCS}, \&$ ExIT $\} \vee|\mathcal{Q}|=0$ in $C$, which continues to hold in $C^{\prime}$. Since Paths $s_{\pi}$ is a set of all maximal paths in $\left(V_{\pi}, E_{\pi}\right)$, there is no path $\sigma \in$ Path $_{\pi}$ for which end $(\sigma)$.PRED $\in\{\&$ InCS, \&EXIT $\}$ and $\operatorname{start}(\sigma)$. PRED $\neq \&$ ExiT. Therefore, the condition holds in $C^{\prime}$.

40. $\pi$ executes Line 40.

In $C, P C_{\pi}=40$, By Condition 27a, $\widehat{\operatorname{node}}_{\pi} \in V_{\pi}$ and by Condition 2, $\widehat{\operatorname{node}}_{\pi}=$ mynode $_{\pi}$. By Condition 23b, all maximal paths in the graph are disjoint, therefore, every vertex in $V_{\pi}$ appears in a unique path in Path $s_{\pi}$.

As argued above, mynode $e_{\pi} \in V_{\pi}$ in $C$, therefore, there is a path $\sigma$ in Path $s_{\pi}$ such that mynode $_{\pi} \in \sigma$. The step sets mypath $_{\pi}$ to be the unique path in Path $s_{\pi}$ in which mynode ${ }_{\pi}$ appears. It then updates $P C_{\pi}$ to 41 .

Condition 33. As argued above, in $C^{\prime}$ mypath $_{\pi}$ is the unique path in Paths $s_{\pi}$ in which mynode $_{\pi}$ appears. Therefore, the condition holds in $C^{\prime}$.

41. $\pi$ executes Line 41.

In $C, P C_{\pi}=41$. By Condition 21, tailpath $_{\pi}=$ NIL in C. By Condition 23b, all maximal paths in the graph are disjoint, therefore, every vertex in $V_{\pi}$ appears in a unique path in Paths $_{\pi}$.

In this step $\pi$ checks if $\operatorname{tail}_{\pi} \in V_{\pi}$. If so, it sets tailpath $_{\pi}$ to be the unique path in Paths $s_{\pi}$ in which tail $_{\pi}$ appears. Otherwise, it just updates $P C_{\pi}$ to $\mathbf{4 2}$.

Condition 28, As argued above, the step sets tailpath $_{\pi}$ to be the unique path in Paths $s_{\pi}$ in which $\mathrm{tail}_{\pi}$ appears. Therefore, the condition holds in $C^{\prime}$.

Condition 31, If tailpath $_{\pi} \neq$ NIL and end $\left(\right.$ tailpath $\left._{\pi}\right)$.PRED $\notin\{\&$ INCS, \&EXIT $\}$, it from Condition 27 that head(fragment $\left(\right.$ tail $\left.\left._{\pi}\right)\right)$.PRED $\notin\{\&$ INCS, \&EXIT $\}$. Hence, the condition follows from Condition 29 . 
42 (a). $\pi$ executes Line 42 when there is a path in Path $s_{\pi}$ not iterated on already.

In $C, P C_{\pi}=42$ and there is a path in $P_{a t h}$ not iterated on already.

In this step $\pi$ picks a path $\sigma_{\pi}$ from Paths $s_{\pi}$ that it didn't iterate on already in the loop on Lines 42,45 . It then sets $P C_{\pi}$ to 43 .

Since no shared variables are changed and no condition of the invariant is affected by the step, all the conditions continue to hold in $C$ as they held in $C^{\prime}$.

42 (b). $\pi$ executes Line 42 when there is no path in $P a t h s_{\pi}$ not iterated on already.

In $C, P C_{\pi}=42$ and there is no path in $P a t h s_{\pi}$ not iterated on already.

In this step $\pi$ finds that it has already iterated on all the paths from $P a t h s_{\pi}$ hence it just updates $P C_{\pi}$ to 46 .

Since no shared variables are changed and no condition of the invariant is affected by the step, all the conditions continue to hold in $C$ as they held in $C^{\prime}$.

43. $\pi$ executes Line 43 .

In $C, P C_{\pi}=43$.

In this step, $\pi$ checks if end $\left(\sigma_{\pi}\right)$.PRED $\in\{\& \operatorname{INCS}, \&$ ExIT $\}$. If so, it updates $P C_{\pi}$ to 44 otherwise it updates $P C_{\pi}$ to 42 .

Condition 36, If $P C_{\pi}=44$ in $C^{\prime}$, it is because of the if condition at Line 43 succeeded. From the description of the step given above, it follows that the condition holds in $C^{\prime}$.

44. $\pi$ executes Line 44.

In $C, P C_{\pi}=44$.

In this step, $\pi$ checks if $\operatorname{start}\left(\sigma_{\pi}\right)$.PRED $\neq \&$ EXIT. If so, it updates $P C_{\pi}$ to 45 , otherwise it updates $P C_{\pi}$ to 42 .

Condition 36. If $P C_{\pi}=45$ in $C^{\prime}$, it is because of the if condition at Line 44 succeeded. Therefore, end $\left(\sigma_{\pi}\right)$.PRED $\in\{\& \operatorname{INCS}, \&$ ExIT $\}$ and $\operatorname{start}\left(\sigma_{\pi}\right)$.PRED $\neq \&$ ExIT. It follows that the length of the path is more than 1 and $\operatorname{start}\left(\sigma_{\pi}\right)=\operatorname{tail}\left(\operatorname{fragment}\left(\operatorname{start}\left(\sigma_{\pi}\right)\right)\right)$. Therefore, from the description of the step given above, it follows that the condition holds in $C^{\prime}$.

45. $\pi$ executes Line 45 .

In $C, P C_{\pi}=45$.

The step sets headpath ${ }_{\pi}=\sigma_{\pi}$ and updates $P C_{\pi}$ to 46 .

Condition 38, This condition follows as a result of Conditions 1, 34, 35, and since the step sets headpath $h_{\pi}=\sigma_{\pi}$.

46 (a). $\pi$ executes Line 46 when tailpat $_{\pi} \neq$ NIL $\wedge$ end $\left(\right.$ tailpat $\left._{\pi}\right)$.PRED $\notin\{\&$ INCS, \&EXIT $\}$.

In $C, P C_{\pi}=46$ and tailpath $_{\pi} \neq$ NIL $\wedge$ end $\left(\right.$ tailpat $\left._{\pi}\right)$. PRED $\notin\{\&$ INCS, \&EXIT $\}$.

In this step $\pi$ checks for the if condition at Line 46 to be met. Since tailpath $_{\pi} \neq$ NIL $\wedge$ end $\left(\right.$ tailpath $\left._{\pi}\right)$.PRED $\notin\{\& \mathrm{INCS}, \&$ ExIT $\}$, the if condition is not met, hence, $\pi$ updates $P C_{\pi}$ to 48 .

Since no shared variables are changed and no condition of the invariant is affected by the step, all the conditions continue to hold in $C$ as they held in $C^{\prime}$.

46 (b). $\pi$ executes Line 46 when tailpat $_{\pi}=$ NIL $\vee$ end $\left(\right.$ tailpat $\left._{\pi}\right)$. PRED $\in\{\&$ InCS, \&EXIT $\}$.

In $C, P C_{\pi}=46$ and tailpath $_{\pi}=$ NIL $\vee$ end $\left(\right.$ tailpat $\left._{\pi}\right)$. PRED $\in\{\&$ INCS, \&EXIT $\}$.

In this step $\pi$ checks for the if condition at Line 46 to be met. Since tailpath $_{\pi}=$ NIL $\vee$ end $\left(\right.$ tailpath $\left._{\pi}\right)$. PRED $\in\{\& \mathrm{INCS}, \&$ ExIT $\}$, the if condition is met, hence, $\pi$ updates $P C_{\pi}$ to 47. 
Since no shared variables are changed and no condition of the invariant is affected by the step, all the conditions continue to hold in $C$ as they held in $C^{\prime}$.

47. $\pi$ executes Line 47 .

In $C, P C_{\pi}=47$.

In this step $\pi$ performs a FAS on TAIL with the node start $\left(\right.$ mypath $\left._{\pi}\right)$ and stores the returned value of the FAS into mypred $_{\pi}$. It then updates $\widehat{P C_{\pi}}$ to $\mathbf{1 4}$ and $P C_{\pi}$ to 49

Condition 39, By Condition 28, fragment $\left(\widehat{\operatorname{node}}_{\pi}\right) \neq$ fragment(TAIL) in $C$. Applying Condition 16 to $C$ and TAIL we see that the condition holds in $C^{\prime}$.

48. $\pi$ executes Line 48 .

In $C, P C_{\pi}=48$.

If headpath $h_{\pi} \neq$ NIL then the step sets mypred $_{\pi}=\operatorname{start}\left(\right.$ headpath $\left._{\pi}\right)$; otherwise it sets mypred $_{\pi}=\&$ SPECIALNODE. The step then updates $\widehat{P C_{\pi}}=\mathbf{1 4}$ and $P C_{\pi}=\mathbf{4 9}$.

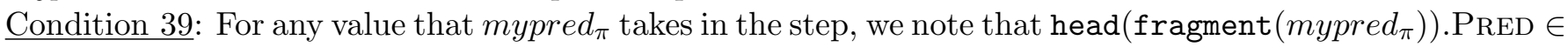
$\{\& \mathrm{INCS}, \& \mathrm{EXIT}\}$. If headpath $\neq$ NIL, then all the parts of the condition are satisfied in $C^{\prime}$ which can be verified from Condition 38 holding in $C$. Also, fragment $\left(\widehat{\operatorname{node}}_{\pi}\right) \neq$ fragment $\left(\right.$ first $\left(\right.$ headpath $\left.\left.h_{\pi}\right)\right)$ in $C^{\prime}$, since head(fragment $\left(\right.$ first $\left(\right.$ headpath $\left.\left.\left._{\pi}\right)\right)\right)$.PRED $\in\{\&$ INCS, \&EXIT $\}$.

If headpath $h_{\pi}=$ NIL then mypred $_{\pi}=\&$ SPECIALNODE in $C^{\prime}$ and it is easy to verify again that the condition holds in $C^{\prime}$. Therefore, the condition holds in $C^{\prime}$.

49. $\pi$ executes Line 49 .

In $C P C_{\pi}=49$.

As a result of the step, mynode $e_{\pi}$.PRED to mypred $_{\pi}$ and updates $\widehat{P C_{\pi}}$ to 25 in $C^{\prime} . \pi$ also executes the Exit section of RLock, hence $P C_{\pi}=\mathbf{2 5}$ in $C^{\prime}$.

The argument for correctness for this step is similar to that of the argument given for execution of Line 14. Therefore, we refer the reader to those arguments above.

Crash. $\pi$ executes a crash step.

This step changes $P C_{\pi}$ to $\mathbf{1 0}$ and sets the rest of the local variables to arbitrary values. The values of the shared variables remain the same as before the crash.

The step does not affect any condition, so the invariant continues to hold in $C^{\prime}$.

Thus, by induction it follows that the invariant holds in every configuration of every run of the algorithm. 